NBER WORKING PAPER SERIES

\title{
WHO WANTS AFFORDABLE HOUSING IN THEIR BACKYARD? AN EQUILIBRIUM ANALYSIS OF LOW INCOME PROPERTY DEVELOPMENT
}

\author{
Rebecca Diamond \\ Timothy McQuade \\ Working Paper 22204 \\ http://www.nber.org/papers/w22204 \\ NATIONAL BUREAU OF ECONOMIC RESEARCH \\ 1050 Massachusetts Avenue \\ Cambridge, MA 02138 \\ April 2016
}

We are grateful to William Diamond, Darrell Duffie, Guido Imbens, Stuart Rosenthal, Amit Seru, and seminar and conference participants at the NBER Summer Institutes of Labor Studies, Public Economics, Urban Economics, and Real Estate, the UCLA Housing Affordability Conference, UCLA economics, UC-Irvine Economics, USC Price, the Federal Reserve Board, and the Stanford GSB economics and finance lunches. The views expressed herein are those of the authors and do not necessarily reflect the views of the National Bureau of Economic Research.

NBER working papers are circulated for discussion and comment purposes. They have not been peer-reviewed or been subject to the review by the NBER Board of Directors that accompanies official NBER publications.

(C) 2016 by Rebecca Diamond and Timothy McQuade. All rights reserved. Short sections of text, not to exceed two paragraphs, may be quoted without explicit permission provided that full credit, including $\odot$ notice, is given to the source. 
Who Wants Affordable Housing in their Backyard? An Equilibrium Analysis of Low Income Property Development

Rebecca Diamond and Timothy McQuade

NBER Working Paper No. 22204

April 2016

JEL No. H23,R13,R38

\begin{abstract}
$\underline{\text { ABSTRACT }}$
We nonparametrically estimate spillovers of properties financed by the Low Income Housing Tax Credit (LIHTC) onto neighborhood residents by developing a new difference-in-differences style estimator. LIHTC development revitalizes low-income neighborhoods, increasing house prices $6.5 \%$, lowering crime rates, and attracting racially and income diverse populations. LIHTC development in higher income areas causes house price declines of $2.5 \%$ and attracts lower income households. Linking these price effects to a hedonic model of preferences, LIHTC developments in low-income areas cause aggregate welfare benefits of \$116 million. Affordable housing development acts like a place-based policy and can revitalize low-income communities.
\end{abstract}

\author{
Rebecca Diamond \\ Graduate School of Business \\ Stanford University \\ 655 Knight Way \\ Stanford, CA 94305 \\ and NBER \\ diamondr@stanford.edu \\ Timothy McQuade \\ Stanford Graduate School of Business School \\ 655 Knight Way \\ Stanford, CA 94305 \\ tmcquade@stanford.edu
}




\section{Introduction}

Increasing geographic income segregation and rising housing costs have put the issue of the government's role in promoting affordable housing at the forefront of current policy debates. ${ }^{1}$ Subsidized housing policy often focuses on easing low income households' housing costs and providing access to financially out of reach neighborhoods. However, subsidized housing is also a place-based policy. Housing subsidies influence households' choices of neighborhoods and developers' choices of where to build. Subsidy induced changes in the locations of households and housing construction can have important spillovers onto the neighborhood residents. ${ }^{2}$ This creates the challenging task of determining how to best allocate affordable housing across neighborhoods. ${ }^{3}$

In this paper, we provide an analysis of the costs and benefits of affordable housing construction to surrounding neighborhood residents and how they vary across demographically different neighborhoods. We study the neighborhood impacts of multifamily housing developments funded through the Low Income Housing Tax Credit (LIHTC). Established in 1986, this program has become an integral component of federal housing policy, funding 21 percent of all multifamily developments over the period 1987-2008. Looking forward, with the construction of publicly run housing projects expected to continue to decline, the LIHTC program is likely to remain one of the main federal government initiatives designed to ensure access to affordable housing by low income households. ${ }^{4}$

We combine data on the location and funding dates for all LIHTC funded projects, housing transaction data from 129 counties, and home buyer race and income data to estimate the effects of LIHTC construction on the surrounding neighborhood. Our estimates show that the impact of affordable housing construction has dramatically different effects on surrounding property values based on whether the affordable housing was built in a relatively richer or poorer neighborhood and whether the neighborhood has a high share of minority residents. LIHTC construction in neighborhoods with a median income below $\$ 26,000$ increases local property values by approximately $6.5 \%$ within 0.1 miles of the development site

\footnotetext{
${ }^{1}$ New York City and San Francisco have both announced plans for large expansions of affordable housing units to "ensure diverse and inclusive neighborhoods." Upon entering office, Bill de Blasio, mayor of New York City, unveiled a plan to create and preserve 200,000 units of affordable housing over ten years. In 2014, the mayor of San Francisco, Ed Lee, outlined an initiative to create 30,000 affordable housing units over six years to "ensure San Francisco remains a place where people from every background can call home.".

${ }^{2}$ Previous research suggests households are willing to pay to live near higher income and more educated neighbors (Bayer et al. (2007); Guerrieri et al. (2014); Diamond (2015)). The quality of the housing stock also spills over onto the value of neighboring houses (Rossi-Hansberg et al. (2010), Campbell et al. (2011)).

${ }^{3}$ Depending on the social planner's preferences, he may want use housing policy to maximize overall social welfare or only the welfare of the low-income population.

${ }^{4}$ Section 8 housing vouchers which provide rental subsidies to low income households who rent in the private rental market is the main alternative federally run low-income housing program.
} 
In contrast, LIHTC construction in neighborhoods with median incomes above $\$ 54,000$ leads to housing price declines of approximately $2.5 \%$ within 0.1 miles of the development site. These declines, however, are only seen in high income areas with a minority population of below $50 \%$.

To account for these price impacts, we explore how LIHTC development affects other characteristics of the local neighborhood, in particular demographics and local crime rates. We find that the construction of a LIHTC development leads to increases in the income of home buyers in low income areas. Conversely, affordable housing development leads to a decrease in home buyer income in higher income areas with low minority populations. Examining the impact of LIHTC construction on the share of Black home buyers, we find that the introduction of affordable housing leads to decreased segregation in lower income areas. Finally, LIHTC development causes declines in both violent and property crime within low income areas, but not does not increase crime in high income areas.

We develop a structural model of housing and neighborhood choice and translate these house price estimates into preference estimates. The average household that desires living near LIHTC sites in low income areas is willing to pay approximately $6 \%$ of their house price to live 0.1 miles from a LIHTC site. In higher income areas with low minority populations, on the other hand, the average household who chooses to live near LIHTC is willing to pay approximately $1.6 \%$ of their total house price to avoid living within 0.1 miles of a LIHTC site. $^{5}$

We use these preference estimates along with census data to calculate the local welfare impact of introducing affordable housing to a particular type of neighborhood. We decompose these effects into the welfare impacts of affordable housing on homeowners, renters, and absentee landlords. ${ }^{6}$ Our analysis reveals large possible societal gains from building affordable housing in low income areas, with construction of LIHTC in low income, low minority areas increasing total welfare by approximately $\$ 116$ million. Building LIHTC in low minority, high income areas leads to losses of approximately $\$ 12$ million.

We identify the effect of affordable housing construction on housing prices by exploiting the timing of when funding is granted for the development along with the exact geographic location of the affordable housing. Clearly the neighborhoods targeted by developers to build affordable housing are non-random. However, the exact geographic location of the

\footnotetext{
${ }^{5}$ While these households who choose to live near LIHTC sites in high income areas dislike LIHTC proximity, they find it optimal to live there since LIHTC proximity also provides a discount on their home prices. On net, these households prefer to live close to LIHTC sites in high income areas than to live further away.

${ }^{6}$ Since we don't have direct data on renters, we assume house prices equal the present discounted value of rents. Further, we assume that renters' preferences for LIHTC proximity are the same as homebuyers, holding race and income fixed.
} 
development site within a broader neighborhood appears to be determined by idiosyncratic characteristics, such as which exact plot of land was for sale at the time. Further, the timing of the funding is often out of the hands of the developer since there is substantial uncertainty in which year the project will be funded. ${ }^{7}$

We harness this identification strategy by developing a new econometric method for estimating a difference-in-differences style estimator in a non-parametric setting where treatment is a smooth function of distance to LIHTC site and time since LIHTC funding. We draw on new methods developed in statistics (Charnigo et al. 2011, Charnigo and Srinivasan, 2015) to transform our data on house price levels to data on the derivative of house prices with respect to distance from LIHTC sites. These transformed data allow us to flexibly difference out very local time trends and neighborhood variation in housing prices. Further, by viewing house prices as a smooth function of geographic location, we show how to generalize discrete geographic fixed effects in house prices to a smooth, time-invariant surface of house prices.

We translate our estimated price effects into households' preferences for living near LIHTC. We employ a structural, generalized hedonic model of housing choice along the lines of Rosen (1974) and Bajari and Benkard (2005) to link observed house prices to individual preferences for proximity to low income housing. The hedonic model allows us to view real estate as a continuous choice of quantities of housing and neighborhood characteristics. We specifically focus on the continuous choice of distance in miles to an LIHTC development. The key advantage of this approach is that it ensures the existence of an equilibrium price surface, which continuously maps housing and neighborhood characteristics to house prices without needing to specify the determinants of housing supply. ${ }^{8}$ As a result of these two key model properties, an agent's optimality condition reduces to a simple equation relating marginal cost of moving further from an LIHTC site to its marginal benefit, which allows us to recover preferences on an individual basis.

The model suggests a straightforward, two-step estimation procedure. First, we nonparametrically estimate the equilibrium pricing surface, i.e. marginal costs, by studying the impact of LIHTC-financed multifamily construction on local house prices. The model's optimality condition then allows us to nonparametrically recover the local gradient of the utility function of each agent. Further parametric assumptions allow for recovery of structural preference parameters and a global description of each agent's utility function. Preferences are then correlated with home buyer demographic information and welfare analysis is

\footnotetext{
${ }^{7}$ Developers must apply for LIHTC funds. Acceptance rates vary across states. In California in 2012, 55 developments were funded from an application pool of 118 .

${ }^{8}$ The existence of a continuous mapping between product characteristics and prices is a feature not shared by standard, discrete-choice models such as a conditional logit models (McFadden, 1973) or Berry, Levionsohn, and Pakes (1995).
} 
conducted.

The heterogeneity of LIHTC impacts across neighborhoods with differing incomes has policy implications when construction of affordable housing is viewed as a place-based policy. Moving LIHTC properties from higher income to lower income neighborhoods benefits both the residents of the higher and lower income neighborhoods. Of course, the neighborhood benefits of LIHTC must be also be weighed against the cost and benefits of the neighborhood to LIHTC tenants. Chetty et al. (2015) find that moving young children from high poverty public housing to low poverty areas increases these childrens' future earnings by a present discounted value of $\$ 100$ thousand. This effect is not large enough to overcome our estimated benefits to low-income neighborhoods simply because there are many more low income households living in a low-income area than in the affordable housing development itself.

Previous work studying the welfare effects of place-based policy subsidizing firm locations has often found it challenging to identify heterogeneous effects of these policies across geographic areas. As stated in Glaeser \& Gottlieb (2011) "For these externalities to create a justification for any particular spatial policy, these externalities must be stronger in some places than in others...Economics is still battling over whether such spillovers exist at all, and we are certainly not able to document compelling nonlinear effects." We are able to both document significant spillovers and heterogeneity in these effects across neighborhoods.

These place-based spillovers due to subsidized housing likely have large economic impacts across the US, as federal, state and local governments spend over $\$ 97$ billion dollars a year on different forms of housing assistance. ${ }^{9}$

A small number of previous studies have examined the impacts of affordable housing on local neighborhoods. Eriksen and Rosenthal (2010) study the crowd-out effects of subsidized affordable housing construction on private rental development. More closely related, BaumSnow and Marion (2009) use census data and a regression discontinuity approach to study the effects of LIHTC financed developments in low income neighborhoods on new construction, median incomes, and property values at the census block group level. They also find that housing prices appreciate in low income areas. Schwartz et al. (2006) look at the price impact of affordable housing in New York City and report positive results. Goujard (2011) performs a similar study of social housing in Paris. Our study leverages extensive micro data to study highly local effects of affordable housing in many different parts of the United States. By looking across a wide array of neighborhoods and counties, we show how affordable housing has dramatically different effects on neighborhood residents based on neighborhood income and the minority share of the neighborhood population. Previous studies either

\footnotetext{
${ }^{9}$ This is more than is spent on unemployment insurance in a typical year.
} 
focus on a single geographic area (Schwartz et al. 2006, Goujard 2011) or only within low income neighborhoods at a single point in time (Baum-Snow and Marion, 2009). Moreover, none of these studies utilize a structural framework in conjunction with detailed data on buyer characteristics to recover and put structure on individual preferences for proximity to affordable housing.

More broadly, our paper is related to a literature which examines the spillovers to neighborhoods of housing policies. Rossi-Hanbserg et al. (2010) study the impact of urban revitalization programs implemented in the Richmond, Virginia area on local land prices. Campbell et al. (2011) examine the effects of housing foreclosure on housing prices nearby. Ellen et al. (2013) look at how foreclosures impact local crime rates. Autor et al. (2014, 2015) study the impact of ending rent control on nearby real estate prices and crime rates.

Finally, a growing literature has found that higher income individuals are willing to pay more for local neighborhood amenities (Bayer et al (2007), Diamond (2015), Handbury (2013)). A number of recent papers have also argued that higher income or more educated neighbors endogenously improve local amenities (Card, Mas, and Rothstein (2008), Bayer et al (2007), Guerrieri et al (2014), Diamond (2015)). However, previous work has not had access to micro-level demographic and housing transaction data. Further, LIHTC development provides a quasi-experimental shock to the income mix of ones' neighbors and allows us to identify the distribution of households' preferences for proximity to low-income neighbors. ${ }^{10}$

The paper proceeds as follows. Section 2 provides institutional background detail about the Low Income Housing Tax Credit. Section 3 describes out data sources and Section 4 details the hedonic model of housing choice. Section 5 discusses our estimation procedure for the pricing surface. We present our reduced form results on price and other neighborhood characteristics in Section 6. Section 7 presents our preference estimates and conducts welfare analysis. Section 8 concludes.

\section{The Low Income Housing Tax Credit}

Since its inception in 1986, the Low Income Housing Tax Credit Program has been an integral component in fostering the development of multifamily housing throughout the United States. With an annual tax credit valued at over 8 billion dollars, the program funded 21 percent of all multifamily developments between the years 1987-2008. Each year, federal tax credits are allocated to the states based on population. To qualify for a tax credit

\footnotetext{
${ }^{10}$ LIHTC development also impacts the overall population density and average age of the neighborhood housing stock. We can't fully seperate out preferences for the income level of one's neighbors from preferences for new construction or increased density. However, the differential value of LIHTC development across neighborhoods of different income levels help zoom in on preferences over the income of one's neighbors.
} 
under the Low Income Tax Credit Program, federal guidelines require that proposed projects must rent to tenants who earn no more than 60 percent of the Area Median Gross Income (AGMI). Additionally, developers must restrict rents, in low-income units to 30 percent of the income limit for a minimum affordability period of 30 years.

Note that these criteria are only the minimal requirements as specified by the federal government. In practice, states almost always receive many more project proposals and tax credit allocation requests from developers than they have federal allotments, generally on the order of 2 to 4 times. Each state is therefore required to maintain a "Qualified Application Plan" (QAP) to govern the selection process. These plans usually operate by assigning point scores to various project characteristics and then allocating tax credits based on point totals until funds are exhausted. See Appendix A.1 for more details.

\section{Data}

We bring together data from a variety of sources. Our first dataset is from DataQuick, which provides detailed public records housing characteristics and transactions data collected from county assessor and register of deeds officers. We restrict our analysis to those counties which have transactions history data dating to at least 1996. This leaves us with a sample of approximately 16 million transactions located within 1.5 miles of a LIHTC site in a total of 129 counties in 15 states. Figure 1 provides a map of the counties in our sample. We merge this dataset with data collected by the United States federal government according to the provisions of the Home Mortgage Disclosure Act (HMDA), which provides us with the race and household income of the homebuyers.

Information on LIHTC financed projects is provided by the Department of Housing and Urban Development (HUD). This data covers 39,094 projects and almost 2,458,000 low income housing units placed into service between the years 1987 and 2012. Our analysis focuses on the 7098 LIHTC projects located in our sample of 129 counties. See Panel B of Table 1 for summary statistics. Due to DataQuick's coverage of counties, our sample is from more dense, urban areas, relative to the overall distribution of LIHTC sites. However, when comparing the characteristics of LIHTC sites developed in urban areas to our 129 county subsample, our sample looks quite representative.

We finally collect 1990 census data at the tract and block group level. These data provide information on median income levels and minority population shares.

Panel A of Table 1 provides summary statistics. Compared to the United States as a whole, the counties in our sample have a similar black share ( $11.6 \%$ vs $12.1 \%$ nationwide), a significantly higher Hispanic share ( $15.3 \%$ vs $8.3 \%$ nationwide), and a median income 
approximately $18 \%$ greater. Median income is higher than the rest of the United States since our sample comprises urban areas and metropolitan areas in relatively high productivity areas such as New England, New York and California. Hispanic share is quite large in our sample since many of our counties are in California and the Southwest, which hosts a large Hispanic population compared to the rest of the country. Within the census block groups which receive LIHTC developments, the Hispanic and Black share are even higher at $24.0 \%$ and $23.6 \%$, respectively. The median incomes are also $33 \%$ lower in these select block groups than average block groups within our sample of counties.

Panel $\mathrm{C}$ of Table 1 also provides summary information about locales within 1 mile, 0.5 miles, 0.2 miles, and 0.1 miles of projects financed through the Low Income Housing Tax Credit program. Average housing prices are about 7\% lower and average home buyer incomes are $5 \%$ lower within half a mile of a LIHTC site than those within 1 mile of an LIHTC site. The percent of home buyers which are Black is $11 \%$ higher within half a mile of an LIHTC site than within one mile. It is clear LIHTC development is targeted at lower income, higher minority share, and lower housing cost areas. However, housing prices, home buyer incomes, and the Black share of home buyers are quite similar between areas within 0.2 miles of an LIHTC site and those within 0.1 miles. The lack of variation in neighborhood characteristics at these more fine geographic measures help substantiate our identification strategy that precise geographic location of LIHTC development provides quasi-experimental variation. See Appendix A.2 for more details on all data sources.

\section{Model of Housing Choice}

Our goal is to formalize a structural, econometric framework which we can use to estimate preferences for proximity to LIHTC properties and thereby quantify the costs and benefits of affordable housing to surrounding neighborhood residents. We develop a generalized hedonic model along the lines of Rosen (1974) and Bajari and Benkard (2005). In this framework, a house $j$ is considered a bundle of characteristics $\left(R_{j}, \mathbf{X}_{j}, \mathbf{Y}_{j}, \boldsymbol{\xi}_{j}\right)$. Here $R_{j} \in \mathbb{R}_{+}$ denotes the distances to the nearest LIHTC property. ${ }^{11}$ The $\left(K_{c}+K_{d}\right)$-dimensional vector $\mathbf{X}_{j}=\left(\mathbf{X}_{j, c}, \mathbf{X}_{j, d}\right) \in \mathbb{R}^{K_{c}} \times \mathbb{R}^{K_{d}}$ denotes physical and location characteristics of the house $j$. The vector $\mathbf{X}_{j, c} \in \mathbb{R}^{K_{c}}$ reflects characteristics which can, to an approximation, be thought of as continuously chosen by agents, such as square-footage, lot size, numbers of beds/baths, and age. The vector $\mathbf{X}_{j, d} \in \mathcal{L}_{\mathbf{X}_{d}} \subset \mathbb{R}^{K_{d}}$, with $\mathcal{L}_{\mathbf{X}_{d}}$ finite, reflects discrete choice variables, such

\footnotetext{
${ }^{11}$ We focus on the choice of proximity to the closest LIHTC site to each house to simplify the model. However, the model can be generalized to allow households to choose to live near multiple LIHTC sites simultaneously.
} 
as whether the property is a condo or single-family house. The $L$-dimensional categorical variable $\mathbf{Y}_{j} \in \mathcal{L}_{\mathbf{Y}} \subset \mathbb{R}^{L}$, with $\mathcal{L}_{\mathbf{Y}}$ finite, reflects neighborhood characteristics of the nearest LIHTC property; in particular, we focus on median income and minority share. This specification allows the agent to view LIHTC properties in different types of neighborhoods as distinct goods with varying impacts on their utility. ${ }^{12}$ Finally, $\boldsymbol{\xi}_{j} \in \mathbb{R}^{J}$ is a $J$-dimensional vector of property and location characteristics of the house which are observable to the home buyer but not to the econometrician. Such variables might include whether there is a finished basement or not. We allow each household to have their own utility function over the housing and neighborhood characteristics: $U_{i}(R, \mathbf{X}, \mathbf{Y}, \boldsymbol{\xi}, c) \cdot{ }^{13} c \in \mathbb{R}_{+}$is a composite good, whose price is normalized to one, which represents all other consumption.

Under this setup, with minimal assumptions on the utility function and no supply side assumptions, Bajari and Benkard (2005) show that there exists an equilibrium price surface which is Lipschitz continuous with respect to characteristics and such that there is a single price for each unique bundle of characteristics. This allow us to write equilibrium house prices as a mapping from characteristics space $p_{j t}=p_{t}(R, \mathbf{X}, \mathbf{Y}, \boldsymbol{\xi})$. Note that the equilibrium price function can vary with time. This is because in the hedonic framework each different time period is treated as a distinct market, in which market primitives such as consumer preferences or marginal production costs can change. ${ }^{14}$

The home buyers' optimization problem can now be written as:

$$
\max _{R, X, Y, \xi, c} U_{i}(R, \mathbf{X}, \mathbf{Y}, \boldsymbol{\xi}, c) \quad \text { such that } \quad p_{t}(R, \mathbf{X}, \mathbf{Y}, \boldsymbol{\xi})+c \leq y_{i}
$$

where $y_{i}$ is the income of agent $i$.

Household $i$ elects his ideal household and neighborhood bundle $\left(R^{*}, \mathbf{X}^{*}, \mathbf{Y}^{*}, \boldsymbol{\xi}^{*}, c^{*}\right)$ by maximizing his utility. For the continuous housing and neighborhood characteristics, this can be written as setting the first order conditions to zero. This relates the slope of households'

\footnotetext{
${ }^{12}$ For example, a given household may find LIHTC proximity desirable when it is built in a low income neighborhood, but undesirable when it is built in a high income neighborhood.

${ }^{13}$ We do not incorporate an additive error term, $\varepsilon_{i j}$, to the utility function which is specific to both the house $j$ and the individual $i$, making this a "pure characteristics model." See Berry \& Pakes (2007) for discussion comparing pure characteristics models to those with product -by-household specific unoberved tastes.

${ }^{14}$ It is important to emphasize that the existence of an equilibrium price surface is not a trivial result which holds in any reasonable model one might write down. In a logit model of demand, for example, two different products with identical characteristics can have different prices if firms have different costs of production. The reason for this is that in logit demand models, in fact any standard discrete-choice model, the existence of the individual specific shock $\varepsilon_{i j}$ ensures that products with different prices but identical characteristics will still both have positive demand. Intuitively, the $\varepsilon_{i j}$ shock means that identical products can't truly exist, in the sense that not everyone in the market will agree on their characteristics. That agents do fully agree on characteristics in the hedonic model imposes structure on the prices.
} 
utility function to the slope of the pricing surface, $p_{t}(R, \mathbf{X}, \mathbf{Y}, \boldsymbol{\xi})$ :

$$
\begin{aligned}
\frac{\partial U_{i}\left(R^{*}, \mathbf{X}^{*}, \mathbf{Y}^{*}, \boldsymbol{\xi}^{*}, c^{*}\right)}{\partial R} & =\frac{\partial p_{t}\left(R^{*}, \mathbf{X}^{*}, \mathbf{Y}^{*}, \boldsymbol{\xi}^{*}\right)}{\partial R} \\
\frac{\partial U_{i}\left(R^{*}, \mathbf{X}^{*}, \mathbf{Y}^{*}, \boldsymbol{\xi}^{*}, c^{*}\right)}{\partial \mathbf{X}_{c}} & =\frac{\partial p_{t}\left(R^{*}, \mathbf{X}^{*}, \mathbf{Y}^{*}, \boldsymbol{\xi}^{*}\right)}{\partial \mathbf{X}_{c}} .
\end{aligned}
$$

Thus, in the case of a continuous choice set, knowledge of the equilibrium price function is sufficient to determine marginal rates of substitution at the chosen bundle.

Affordable housing proximity may be viewed as an amenity in some areas, but a disamenity in others. To ensure the utility function is always concave when it is increasing, we use slightly different functional forms to represent utility when LIHTC proximity is viewed as desirable versus undesirable. We parameterize households' preference for proximity to LIHTC developments as:

$$
\begin{aligned}
U_{i}^{G}(R, \mathbf{X}, \mathbf{Y}, \boldsymbol{\xi}, c) & =\gamma_{i, \mathbf{Y}} \log \left(1+R_{0, \mathbf{Y}}-R\right) \mathbf{1}\left[R \leq R_{0, \mathbf{Y}}\right]+u_{i}(\mathbf{X}, \boldsymbol{\xi})+c \\
U_{i}^{B}(R, \mathbf{X}, \mathbf{Y}, \boldsymbol{\xi}, c) & =\gamma_{i, \mathbf{Y}} \log \left(\frac{1+R}{1+R_{0, \mathbf{Y}}}\right) \mathbf{1}\left[R \leq R_{0, \mathbf{Y}}\right]+u_{i}(\mathbf{X}, \boldsymbol{\xi})+c,
\end{aligned}
$$

where $U_{i}^{G}(R, \mathbf{X}, \mathbf{Y}, \boldsymbol{\xi}, c)$ represents utility when living closer to LIHTC is viewed as desirable and $U_{i}^{B}(R, \mathbf{X}, \mathbf{Y}, \boldsymbol{\xi}, c)$ represents utility when living closer to LIHTC is viewed as undesirable. Here, $R$ is the distance in miles to the nearest LIHTC property, $R_{0, \mathbf{Y}}$ is the maximal distance at which proximity to LIHTC of type $\mathbf{Y}$ contributes to agent utility. If an agent lives more than $R_{0, \mathbf{Y}}$ from an LIHTC site, his utility is not impacted. This functional form ensures utility is always concave when it is increasing and that the utility value of living at distance $R_{0, \mathbf{Y}}$ is equal to zero regardless of whether LIHTC proximity is viewed as desirable or undesirable. The parameter $\gamma_{i, \mathbf{Y}}$ reflects agent $i$ 's personal preference for proximity to LIHTC in neighborhood of type Y. Note that individual households view LIHTC properties in different types of neighborhoods as distinct goods and can have varying preferences with respect to them. The function $u_{i}(\mathbf{X}, \boldsymbol{\xi})$ reflects the utility contribution of the house's physical and location characteristics.

We place minimal restrictions on the equilibrium price function. First, we assume that for any $\mathbf{Y} \in \mathcal{L}_{\mathbf{Y}}$, there exists some distance $R_{0, \mathbf{Y}}$, beyond which LIHTC proximity no longer impacts prices: ${ }^{15}$

$$
\text { if } R>R_{0, \mathbf{Y}}: \frac{\partial p_{t}(R, \mathbf{X}, \mathbf{Y}, \boldsymbol{\xi})}{\partial R}=0 \text {. }
$$

If house prices are increasing with distance to LIHTC, then standard optimization implies

\footnotetext{
${ }^{15}$ In the estimation, we will identify $R_{0, \mathbf{Y}}$ by finding the point at which the price gradient is estimated to equal 0 .
} 
that the location choice of agent $i$ with utility $U_{i}^{B}$ who dislike proximity to LIHTC housing will satisfy the interior first-order condition if $R^{*}<R_{0, \mathbf{Y}}:^{16}$

$$
\text { if } R^{*}<R_{0, \mathbf{Y}}: \frac{\gamma_{i, \mathbf{Y}}}{1+R_{i}^{*}}=\frac{\partial p_{t}\left(R_{i}^{*}, \mathbf{X}_{i}^{*}, \mathbf{Y}_{i}^{*}, \boldsymbol{\xi}_{i}^{*}\right)}{\partial R}
$$

Conversely, in areas in which house prices are decreasing with distance from LIHTC developments, individuals with utility $U_{i}^{G}$ who like proximity to affordable housing will satisfy the interior first-order condition if $R^{*}<R_{0, \mathbf{Y}}$ :

$$
\text { if } R^{*}<R_{0, \mathbf{Y}}: \frac{-\gamma_{i, \mathbf{Y}}}{1+R_{0, \mathbf{Y}}-R_{i}^{*}}=\frac{\partial p_{t}\left(R_{i}^{*}, \mathbf{X}_{i}^{*}, \mathbf{Y}_{i}^{*}, \boldsymbol{\xi}_{i}^{*}\right)}{\partial R}
$$

Individuals who dislike proximity to LIHTC will move out of the area, satisfying $R_{i}^{*}>R_{0, \mathbf{Y}}$.

The above suggests a two-step procedure for recovering households' preferences for LIHTC proximity. First, estimate the equilibrium price function, and in particular its gradient with respect to LIHTC proximity in each neighborhood type Y. Next, each households' observed choice of LIHTC proximity $R_{i}^{*}$ and the estimated gradient function are used to identify the household's preference for LIHTC proximity $\gamma_{i, \mathbf{Y}}$ from equations (7) and (8) .

\section{Estimating the Equilibrium Price Function}

The minimal set of assumptions outlined in the previous section do not provide much guidance to the actual form of the equilibrium price function. It ultimately depends on a variety of inputs, such as marginal costs and the nature of competition in the housing market which are not modeled. In particular, we suppose:

$$
\log p_{t}(R, \mathbf{X}, \mathbf{Y}, \boldsymbol{\xi})=\widetilde{m}_{\mathbf{Y}}(R, \tau)+h_{t}(\mathbf{X}, \boldsymbol{\xi})
$$

where $\widetilde{m}_{\mathbf{Y}}(R, \tau)$ represents the impact of being $R$ miles away from an LIHTC development built in neighborhood type $\mathbf{Y}$. We allow the price impact of LIHTC development to vary with $\tau$, the number of years since the LIHTC site received government funding. We assume this price impact of LIHTC development is additively separable from the function $h_{t}(\mathbf{X}, \boldsymbol{\xi})$ which measures the price impacts of the other physical and neighborhood characteristics. We are interested in estimating the gradient of $\widetilde{m}_{\mathbf{Y}}(R, \tau)$ with respect to $R$.

To develop a formal econometric model, we first index all of the LIHTC locations by a

\footnotetext{
${ }^{16}$ Individuals of type $G$ may also live in these neighborhoods who value proximity to LIHTC housing. Since prices are decreasing as one moves closer to the LIHTC site in these neighborhoods, such individuals will optimally locate themselves at the corner solution $R_{i}^{*}=0$.
} 
unique $l$. Each location $l$ will have a type $\mathbf{Y} \in \mathcal{L}_{\mathbf{Y}}$. We refer to neighborhood $l$ as the 1.5 mile radius circle surrounding LIHTC site $l$. We refer to each geographic location within neighborhood $l$ by its polar coordinates $(r, \theta)$, relative to the location of the LIHTC site. ${ }^{17,18}$ Since housing and neighborhood characteristics $(\mathbf{X}, \boldsymbol{\xi})$ vary with the geographic location of the house $(r, \theta)$ and the year $t$, there is a mapping from geographic location and time $(r, \theta, t)$ to the available characteristics $(\mathbf{X}, \boldsymbol{\xi})$ at that location:

$$
(\mathbf{X}, \boldsymbol{\xi})=g_{l}(r, \theta, t)
$$

where $g_{l}$ is a unique vector of functions for each neighborhood $l$. Plugging this into equation (9):

$$
\log p_{t}\left(r, g_{l}(r, \theta, t), \mathbf{Y}\right)=\widetilde{m}_{\mathbf{Y}}(r, \tau)+h_{t}\left(g_{l}(r, \theta, t)\right)
$$

If LIHTC development was randomly assigned to geographic locations, then equation (10) could be estimated by treating $h_{t}\left(g_{l}(r, \theta, t)\right)$ as a residual and non-parametrically estimating the relationship between housing prices and distance to the LIHTC site $R$ and years since LIHTC funding $\tau$. However, clearly LIHTC developers target developments to certain types of neighborhoods and our estimator needs to account for this. To do so, we develop a difference-in-differences style setup to identify $\widetilde{m}_{\mathbf{Y}}(R, \tau)$. We estimate the econometric specification:

$$
\log p_{j t}=\widetilde{m}_{\mathbf{Y}}\left(r_{j}, \tau_{j}\right)+\phi_{l}\left(r_{j}, \theta_{j}\right)+\varphi_{l}\left(\theta_{j}, t_{j}\right)+\varepsilon_{j t} .
$$

The non-parametric function $\phi_{l}\left(r_{j}, \theta_{j}\right)$ denote location "fixed effects." In a standard differencein-difference framework, treatment is usually assigned to discrete entities (e.g. people, cities, firms), however we generalize this to the continuous case and allow for a smooth surface of housing prices across geographic locations that do not depend on time. ${ }^{19}$ The non-parametric function $\varphi_{l}\left(\theta_{j}, t_{j}\right)$ allows for a time trend for neighborhood $l$, which also could potentially vary based on $\theta .{ }^{20}$ We have assumed that local price trends do not depend on $r$. $\varepsilon_{j t}$ captures

\footnotetext{
${ }^{17}$ Note that by defining geographic locations in polar coordinates $(r, \theta)$ relative to the LIHTC site $l$, Distance to the LIHTC $R$, is equal to $r$, the polar coordinate radius coordinate. Thus, $R=r$.

${ }^{18}$ We denote the probability density of house locations as $f(r, \theta, t)$. We make the technical assumption that $f(\theta \mid r, t)=f\left(\theta \mid r, t^{\prime}\right)$ for any $t \neq t^{\prime}$.

${ }^{19}$ One could consider discretizing the geography into something like census tracts or even individual houses and including census tract or house fixed effects in the regression. However, this suffers from a bias/variance tradeoff where if the geographic units are too large, the regression does not adequately control for neighborhood variation in house prices. However if the geographic units are too small (house fixed effects) a large amount of information is thrown away. In the case of house fixed effects, we would only be able to use repeat sales of the same houses to identify our estimates, even if there were many houses that only transacted once but were located right next to each other. The smooth surface of housing prices over geography attempts to deal with this bias/variance tradeoff more efficiently.

${ }^{20}$ For example, it could be that in some neighborhoods there is more house price appreciate to the north than to the south for reasons unrelated to LIHTC development. While not controlling for $\theta$ is unlikey to
} 
that some housing and neighborhood characteristics will vary discretely over geographic location and time, and thus will not be captured by $\tilde{m}_{\mathbf{Y}}\left(r_{j}, \tau_{j}\right)+\phi_{l}\left(r_{j}, \theta_{j}\right)+\varphi_{l}\left(\theta_{j}, t_{j}\right) .^{21}$

To identify equation (11) we assume that local housing price trends unaccounted for by our location "fixed effects" $\phi_{l}\left(r_{j}, \theta_{j}\right)$ and neighborhood time trends $\varphi_{l}\left(\theta_{j}, t_{j}\right)$ are independent of distance to the LIHTC site, $r_{j}$, and year since LIHTC funding, $\tau_{j}$ :

$$
E\left(\varepsilon_{j t} \mid \widetilde{m}_{\mathbf{Y}}\left(r_{j}, \tau_{j}\right), \phi_{l}\left(r_{j}, \theta_{j}\right), \varphi_{l}\left(\theta_{j}, t_{j}\right)\right)=0 .
$$

While the overall neighborhood is likely an endogenous choice by developers, due to highly local supply constraints such as the exact location of available lots, the placement and timing of a low-income property is plausibly exogenous with respect to highly local price trends. Intuitively, we can obtain a consistent estimate of the non-parametric price gradient by examining price changes close to a LIHTC property vs price changes slightly further away and then using differences-in-differences to "difference out fixed effects". Furthermore, to the extent that the treatment effect ultimately decays towards zero with distance within this area, we can estimate the overall level treatment effect by integrating our estimate of the gradient.

While we set up the estimator in non-parametric framework, such a strategy has been pursued in previous papers, albeit in a more parametric form. This more parametric approach would compare price changes in an inner circle of certain radius to a price changes in an outer ring of certain radius that surrounds the inner ring. The inner ring would be thought to receive the treatment, while the outer ring would act as the control. Examples of this approach include Currie et al. (2013), Autor et al. (2014), Aliprantis and Hartley (2014), and Shoag and Veuger (2015). ${ }^{22}$ The advantage of our framework is that our results are not sensitive to the choice of inner ring and outer ring radii and it allows for a more granular estimate of the decay of the treatment effect with distance.

Standard estimation methods for estimating additively separable non-parametric functions, as we have in equation (11) are very computationally challenging to work with when there are many additively separable non-parametric functions. ${ }^{23}$ Instead, we build on new

bias our estimates, it can help with regard to efficiency.

${ }^{21}$ For example, crossing a school district boundary will lead to a discountinuous jump in school quality, which will not be fully picked up by $\phi_{l}\left(r_{j}, \theta_{j}\right)$, since we assume $\phi_{l}\left(r_{j}, \theta_{j}\right)$ is continuous.

${ }^{22}$ Autor et al. (2014) approachs this problem slightly differently using exponential weighting based on distance.

${ }^{23}$ The standard method is a procedure called back-fitting which is a Gauss-Seidel algorithm where one estimates an individual non-parametric function, given a guess of the other additively seperable non-parametric functions. The procedure loops overs each non-paramteric fucntion, given the best guess of the others until the method converges. See Hastie and Tibshirani (1990) for more details. This method would be incredibly computationally challenging as we would have to estimate 2 non paramteric functions for each LIHTC site 
methods developed in the statistics literature by Charnigo et al. (2011) and Charnigo and Srinivasan (2015) which allow us to directly estimate the derivative of $\widetilde{m}_{\mathbf{Y}}\left(r_{j}, \tau_{j}\right)$ allowing us to "difference out" some of additively separable nonparametric functions, which makes estimation computationally feasible. The idea is to estimate gradients of the pricing surface using empirical partial derivatives and then to use kernel regression as a smoothing procedure. The key advantage of these methods is that they provide a way to "difference out" the fixed effects and time trends in a spatial, non-parametric setting, just as one would do in a fully parametric differences-in-differences design. The procedure provides substantial computational and efficiency gains over the alternative of estimating the full non-parametric surface in levels and then taking derivatives. We outline the methodology here.

First, we create "empirical derivatives" of the log house price surface with respect to distance $r$ to the LIHTC site at each housing transaction data point $\left(r_{\iota}, \theta_{\iota}, t_{\iota}\right)$. Let $\tilde{Y}_{\iota}$ be the empirical derivative at $\left(r_{\iota}, \theta_{\iota}, t_{\iota}\right)$ within neighborhood $l$ constructed according to:

$$
\begin{aligned}
\tilde{Y}_{\iota, l} & =\sum_{k=1}^{\kappa_{n}} w_{k} \frac{\log p_{a(k, \iota, r)}-\log p_{b(k, \iota, r)}}{r_{a(k, \iota, r)}-r_{b(k, \iota, r)}} \\
w_{k} & =\frac{k}{\kappa_{n}\left(\kappa_{n}+1\right) / 2}
\end{aligned}
$$

with the observation subscripts recursively defined by:

$$
\begin{aligned}
& a(1, \iota, r)=\arg \min _{\left\{d \in L_{r, \iota}: r_{d}>r_{\iota}+l_{n}\right\}} r_{d}, \quad b(1, i, r)=\arg \underset{\left\{d \in L_{r, i}: r_{d}<r_{i}-l_{n}\right\}}{\max } r_{d} \\
& a(k, \iota, r)=\arg \min _{\left\{d \in L_{r, \iota} r_{d}>r_{a(k-1, \iota, r)}\right\}} r_{d}, \quad b(k, i, r)=\arg \underset{\left\{d \in L_{r, \iota}: r_{d}<r_{b(k-1, \iota, r)}\right\}}{\max } r_{d}
\end{aligned}
$$

where $l_{n}>0 .^{24}$

$$
L_{r, i}:=\left\{p \in\{1, \ldots, n\}: \frac{\left(t_{p}-t_{i}\right)^{2}}{\left(r_{p}-r_{i}\right)^{2}}<\vartheta_{n}^{t}, \frac{\left(\theta_{p}-\theta_{i}\right)^{2}}{\left(r_{p}-r_{i}\right)^{2}}<\vartheta_{n}^{\theta}\right\}
$$

Equation (12) calculates a numerical derivative with respect to LIHTC distance between a pair of houses $a(k, \iota, r)$ and $b(k, \iota, r)$ by the difference in house prices $\left(\log p_{a(k, \iota, r)}-\log p_{b(k, \iota, r)}\right)$ divided by the difference in LIHTC distance $\left(r_{a(k, \iota, r)}-r_{b(k, \iota, r)}\right)$. Equation (12) calculates these numerical derivatives for $\kappa_{n}$ pairs of houses and creates a weighted average of these with weights $w_{k}$. This weighted average is our empirical derivative at $\left(r_{\iota}, \theta_{\iota}, t_{\iota}\right), \widetilde{Y}_{\iota}$.

$l$ along with $\widetilde{m}_{\mathbf{Y}}\left(r_{j}, \tau_{j}\right)$. This would require iterating over more than 14,000 functions.

${ }^{24} l_{n}$ ensures we throw away house price transactions extremely close to $r_{\iota}$. If we have numerical derivatives from transactions which occur at the exact same location as $r_{i}$ (such as multiple transactions in the same condo building), the denominator in equation (12) will blow up. Thus, we throw out transaction less then $l_{n}=0.01$ miles away from $r_{i}$. 
Equations (14), (15), and (16) determine which houses to use for the numerical derivative calculation. Equation (16) first determines the set of "eligible" houses to use in the empirical derivative. Since we are interested in the derivative with respect to LIHTC distance, we ideally would want to compute the empirical derivatives using houses with identical $\theta$ s and $t$ s. Essentially, we want to hold $\theta$ and $t$ fixed and zoom in on house price variation only in the $r$ dimension. Since we cannot choose where to observe house price transactions, we create a tolerance window within which $\theta$ and $t$ are "approximately" held fixed. Equation (16) states that houses are in the set eligible for our empirical derivative calculation if both their squared distance in time from $t_{i}$ and squared distance in angle from $\theta_{i}$ is no more than $\vartheta_{n}^{t}$ and $\vartheta_{n}^{\theta}$,respectively, their squared distance in LIHTC distance $r_{i}$. Essentially this means that houses in the eligible set $L_{r, i}$ are similar to $\left(r_{\iota}, \theta_{\iota}, t_{\iota}\right)$ in the $\theta$ and $t$ dimension, but differ in the $r$ dimension. Visually, this can be thought of house transactions which fall within a 3D "bowtie" around $\left(r_{\iota}, \theta_{\iota}, t_{\iota}\right)$, as illustrated in Figure 2. $\vartheta_{n}$ is a tuning parameter which determines the width of the bowtie. A large $\vartheta_{n}$ allows more house prices to be eligible for the empirical derivative calculation, but will also add bias since they will have more variation in the $\theta$ and $t$ dimension. A smaller $\vartheta_{n}$ allows less data to be used in the empirical derivative calculation, leading to more variance. We search for (at most) $\kappa_{n}$ nearby transactions within this "bowtie" of nearby locations. ${ }^{25}$

The house prices $(a(1, \iota, r), \ldots, a(k, \iota, r))$ are transactions which are further away from the LIHTC site than $r_{\iota}$ and are ordered by distance from $r_{\iota}$. Thus, $a(1, \iota, r)$ is closer to $r_{\iota}$ than $a(2, \iota, r)$. Similarly, the house prices $(b(1, \iota, r), \ldots, b(k, \iota, r))$ are the transactions closer to the LIHTC site than $r_{\iota}$ and are ordered by proximity to $r_{\iota}$. Thus, $b(1, \iota, r)$ is closer to $r_{\iota}$ than $b(2, \iota, r)$. To construct the empirical derivatives, equations (14), (15) state that the housing transactions are paired together based on how close they are to $r_{\iota}$. Thus, the first pair of houses used in the empirical derivative calculation would be $a(1, \iota, r)$, the closest house to $\left(r_{\iota}, \theta_{\iota}, t_{\iota}\right)$ and further from the LIHTC site than $\left(r_{\iota}, \theta_{\iota}, t_{\iota}\right)$, and $b(1, \iota, r)$, the closest house to $\left(r_{\iota}, \theta_{\iota}, t_{\iota}\right)$ which closer to the LIHTC. Note that these houses must also fall within the bowtie tolerance region defined by equation (16). When constructing empirical derivatives in each local area, we use only pre-treatment data in constructing pre-treatment derivatives and only post-treatment data in constructing post-treatment derivatives. This is to ensure that data from the post-treatment period has no effect on the pre-treatment estimates.

Once we have transformed our data on house price levels to data on house price derivatives, we smooth these house price derivatives using a standard kernel estimator. Define the

\footnotetext{
${ }^{25}$ We use $\kappa_{n}$ as the maximum number of house price pairs included in the calculation. However, sometimes there is less than $\kappa_{n}$ pairs of house prices in the bowtie region. In this case, we use as many pairs as there are available in the data. Formally, the number of available house price pairs is a random variable. We address this in the econometric proofs in the appendix.
} 
Nadaraya-Watson kernel estimate at $(r, t)$ :

$$
\widehat{\Phi}_{l}(r, t)=\frac{n^{-1} \sum_{\iota=1}^{n} K_{\mathbf{H}_{n}}\left((r, t)-\left(r_{\iota}, t_{\iota}\right)\right) \tilde{Y}_{\iota, l}}{n^{-1} \sum_{\iota=1}^{n} K_{\mathbf{H}_{n}}\left((r, t)-\left(r_{\iota}, t_{\iota}\right)\right)}
$$

where:

$$
K_{\mathbf{H}_{n}}\left((r, t)-\left(r_{\iota}, t_{\iota}\right)\right)=\frac{1}{h_{r, n} h_{t, n}} K\left(\frac{r-r_{\iota}}{h_{r, n}}, \frac{t-t_{\iota}}{h_{t, n}}\right)
$$

and $K(\cdot, \cdot)$ is the two-dimensional Epichanokov kernel with bandwidths $h_{r, n}, h_{t, n}$. for $\vartheta_{n}>0$. Thus, $\widehat{\Phi}_{l}(r, t)$ is constructed around each LIHTC site $l$.

The following theorem provides a consistent estimate of the gradient treatment effect.

\section{Theorem 1 Suppose:}

1. $n \rightarrow \infty, h_{n} \rightarrow 0, l_{n} \rightarrow 0, \kappa_{n} \rightarrow \infty, \vartheta_{n} \rightarrow 0$

2. $n h_{n} \rightarrow \infty, \kappa_{n} /\left(n \vartheta_{n}^{2}\right) \rightarrow 0, l_{n}^{2} \kappa_{n} \rightarrow \infty$

Letting $T_{l}$ denote the treatment year of LIHTC site l of type $\mathbf{Y}$. Then the following:

$$
\widehat{\Phi}_{l}\left(r, T_{l}+\tau\right)-\widehat{\Phi}_{l}\left(r, T_{l}-1\right)
$$

is a consistent estimate of $\frac{\partial \widetilde{m}_{\mathbf{Y}}(r, \tau)}{\partial r}$, where we assume $\frac{\partial \widetilde{m}_{\mathbf{Y}}(r,-1)}{\partial r}=0$.

The proof of this result, as well as a general discussion of nonparametric derivative estimation in both the univariate and multivariate setting, are provided in the Appendix B. This result generalizes Charnigo et al. (2011) and Charnigo and Srinivasan (2015) to the case where the data are observed at random locations. Charnigo et. al (2011) analyze a univariate case when the data can be observed at chosen locations. Charnigo and Srinivasan (2015) analyze the multivariate case where the locations of the data are again fixed. Neither of these consider the random design, difference in differences setup.

In our empirical work, we define the treatment year $T_{l}$ as the year in which funds are allocated for the development project, rather than the year the project is placed in service. We do this for two reasons. First, prices are forward looking and thus should reflect anticipated neighborhood effects of low income property development when the project is announced. Second, the construction of the project itself may have direct effects on prices prior to the development being placed into service, but after the funding is announced. We set $\kappa_{n}=5$ (the number of house pair used in each empirical derivative), $h_{r, n}=0.3$ (the bandwidth for smoothing in miles), $h_{t, n}=5$, (the bandwidth for smoothing in years), $\vartheta_{n}^{t}=1.6$ (the bow-tie width in years), $\vartheta_{n}^{\theta}=0.4$ (the bow-tie width in distance perpendicular to $r$ ), and $l_{n}=.01$ 
(miles around the empirical derivative location to drop data) ${ }^{26}$. With enough house price transactions around a single LIHTC site, we would be able to estimate the price impacts for each site individually, however for power reasons we average our estimates across LIHTC sites of type $\mathbf{Y}$ :

$$
\frac{\partial \widetilde{m_{\mathbf{Y}}(r, \tau)}}{\partial r}=\frac{1}{N_{\mathbf{Y}}} \sum_{l \in \mathbf{Y}}\left[\widehat{\Phi}_{l}\left(r, T_{l}+\tau\right) d t-\widehat{\Phi}_{l}\left(r, T_{l}-1\right)\right]
$$

where $N_{\mathbf{Y}}$ is the number of LIHTC sites of type $\mathbf{Y}$ in our data. We use block bootstrapping over LIHTC neighborhoods to obtain standard errors.

\section{Reduced Form Results}

\subsection{Price Effects}

We begin by studying the reduced form price effects. For clarity of exposition, we begin by presenting the nonparametric level estimates obtained by integrating the gradient estimates as described in the previous section. Figure 3 illustrates the average impact of LIHTC construction on local house prices across all neighborhoods. First, note that prices leading up to the LIHTC funding are quite flat, validating our identification assumption that absent LIHTC construction, housing prices very close to the LIHTC site would have trended similarly to house prices slightly further away. Nonetheless, it appears from Figure 3 that LIHTC construction has no significant average impact on local house prices. However, this figure masks substantial heterogeneity in the price impact of LIHTC development on local house prices.

To examine such heterogeneity, we re-estimate the price effects for construction in various location types $\mathbf{Y} \in \mathcal{L}_{\mathbf{Y}}$. We begin by dividing the LIHTC sites into four buckets based on the 1990 census median income of the census block group in which the LIHTC site is located. The income quartile cutoffs are $\$ 26,017, \$ 38,177$, and $\$ 54,642$ in 2012 dollars. Note that, consistent with the summary statistics evidence provided in Table 1, the cutoff for the top income quartile is still substantially below the average block group median income of $\$ 66,652$ for the counties in our sample. Moreover, LIHTC residents must earn no more than $60 \%$ of the local area's median gross income, which on average across all our counties is $0.6 * \$ 66,652$

\footnotetext{
${ }^{26} l_{n}$ is required since something there can be transactions at the exact same location (in a condo building) and the distance between the transaction is zero. This is not useful for the empirical derivative calculations since the denominator of the empirical derivatives (distance between the transactions) would be zero. The need to drop data very very close to the site of the empirical derivative shows up in the consistency proofs in the appendix as well.
} 
$=\$ 39,991$. Thus, the bottom quartile of LIHTC sites have residents earning significantly below the average income cutoff, while the top quartile neighborhoods have median incomes about $\$ 15,000$ above the average income cutoff. ${ }^{27}$

Figure 4 illustrates the heterogeneity in price impacts. Panel A shows that LIHTC construction triggers large local price appreciation of approximately $6.5 \%$ after 10 years in the bottom income quartile. Panel B shows that LIHTC development has little impact in the second income quartile, beyond maybe small appreciation very close to the development site. We see in panels $\mathrm{C}$ and $\mathrm{D}$ that construction of affordable housing leads to striking and markedly different effects in both the third and fourth income quartiles, with construction leading to price declines of approximately $3 \%$ after 10 years in the third income quartile and declines of $2.5 \%$ in the fourth income quartile. The speed of the price decline in the fourth income quartile is dramatic, with practically all losses within 0.1 miles of a LIHTC site over the 10 year period occurring in the first year. However, the price declines in income quartiles 3 and 4 "radiate outwards" as time since LIHTC funding increases. At distances of 0.3 to 0.4 miles away from the LIHTC site, there are modest declines in house prices right away, but they fall over time. It appears the housing market very quickly "prices" the impact of LIHTC very locally, but it takes 5 to 10 years for the house prices 0.3 to 0.4 miles away to fully adjust to the shock. In all cases, we do not see strong evidence for pre-trends in prices, further validating our identification assumption that there are no very local house price trends correlated with LIHTC development.

We additionally examine the impact of LIHTC development in high minority areas. In particular, we restrict to those LIHTC sites located within a census block group that has a population at least 50\% Black or Hispanic based on the 1990 census. We then further classify these sites based on whether they are in low income areas, defined as within the first or second income quartile, or high income areas, defined as within the third or fourth income quartiles.

Figure 5 illustrates the effects of affordable housing construction in high minority areas. Low income, high-minority areas see strong price appreciation of approximately $5 \%$ after 10 years resulting from LIHTC development, similar to the overall effect we see in the first and second income quartile. Conversely, prices in high income, high-minority areas remain relatively stable, with no evidence of the house price decline documented above. Thus, the substantial price depreciation seen in high income areas occurs in those neighborhoods with minority populations of below $50 \%$.

We define the short term effect as the average price gradient impact on LIHTC site $l$ between event years 0 through 5 , relative to event years -5 to 0 :

\footnotetext{
${ }^{27}$ This is an approximate, back of the envelope calcuation. AGMI limits vary county by county.
} 


$$
\frac{\partial \log p^{\text {short }}}{\partial r}(r, l)=\frac{1}{5} \int_{0}^{5} \widehat{\Phi}_{l}\left(r, T_{l}+\tau\right) d \tau-\frac{1}{5} \int_{-5}^{0} \widehat{\Phi}_{l}\left(r, T_{l}-\tau\right) d \tau .
$$

Similarly we define the longer term impact of LIHTC sites as the impact in event years 5 through 10 , relative to event years -5 to 0 :

$$
\frac{\partial \log p^{\text {long }}}{\partial r}(r, l)=\frac{1}{5} \int_{5}^{10} \widehat{\Phi}_{l}\left(r, T_{l}+\tau\right) d \tau-\frac{1}{5} \int_{-5}^{0} \widehat{\Phi}_{l}\left(r, T_{l}-\tau\right) d \tau .
$$

We decompose these price gradient effects into differential effects based on income quartile and minority share. We define $\frac{\partial \widetilde{m}_{\mathbf{Y}}^{\text {short }}\left(r_{j}\right)}{\partial r}$ and $\frac{\partial \widetilde{m}_{\mathbf{Y}}^{\text {long }}\left(r_{j}\right)}{\partial r}$ as the short term (within 5 years) and long term (6-10 years) gradients of the price effect with respect to distance $(r)$ of LIHTC development in neighborhoods of type $\mathbf{Y}$ :

$$
\begin{aligned}
& \frac{\partial \log p^{\text {short }}(r, l)}{\partial r}=\frac{\partial \widetilde{m}_{\mathbf{Y}}^{\text {short }}\left(r_{j}\right)}{\partial r}+\varepsilon_{r l}^{\text {short }}, \\
& \frac{\partial \log p^{\text {long }}(r, l)}{\partial r}=\frac{\partial \widetilde{m}_{\mathbf{Y}}^{\text {long }}\left(r_{j}\right)}{\partial r}+\varepsilon_{r l}^{\text {long }} .
\end{aligned}
$$

We allow the neigbhorhood price effects to vary by the income quartile of the neighborhood $\left(\mathbf{Y}_{l}^{\text {inc }}\right)$ and whether the neighborhood has a high minority share $\left(\mathbf{Y}_{l}^{\text {minor }}\right)$ :

$$
\begin{aligned}
& \frac{\partial \widetilde{m}_{\mathbf{Y}}^{\text {short }}\left(r_{j}\right)}{\partial r}=\delta_{\mathbf{Y}_{l}^{\text {inc }}}^{\text {shor }}(r)+\beta_{\mathrm{m} \text { low }}^{\text {short }}(r) * \mathbf{Y}_{l}^{\text {minor }} * \mathbf{1}\left[\mathbf{Y}_{l}^{\text {inc }} \leq 2\right]+\beta_{\mathrm{m} \mathrm{high}}^{\text {short }}(r) * \mathbf{Y}_{l}^{\text {minor }} * \mathbf{1}\left[\mathbf{Y}_{l}^{\text {inc }}>2\right], \\
& \frac{\partial \widetilde{m}_{\mathbf{Y}}^{\text {long }}\left(r_{j}\right)}{\partial r}=\delta_{\mathbf{Y}_{l}^{\text {inc }}}^{\text {long }}(r)+\beta_{\mathrm{mlow}}^{\text {long }}(r) * \mathbf{Y}_{l}^{\text {minor }} * \mathbf{1}\left[\mathbf{Y}_{l}^{\text {inc }} \leq 2\right]+\beta_{\mathrm{mhigh}}^{\text {long }}(r) * \mathbf{Y}_{l}^{\text {minor }} * \mathbf{1}\left[\mathbf{Y}_{l}^{\text {inc }}>2\right] .
\end{aligned}
$$

where $\mathbf{Y}_{l}^{\text {inc }} \in[1,2,3,4]$ is the income quartile of the neighorbhood surrounding LIHTC site 1 and $\mathbf{Y}_{l}^{\text {minor }}$ is an indicator variable equal to 1 if LIHTC site is located in a high minority area. $\delta_{\mathbf{Y}_{l}^{\text {inc }}}^{\text {short }}(r)$ and $\delta_{\mathbf{Y}_{l}^{\text {inc }}}^{\text {long }}(r)$ represent the short and long term price gradient impacts of LIHTC development in low minority areas in income quartile $\mathbf{Y}_{l}^{\text {inc }}$ at distance r. $\beta_{\text {mlow }}^{\text {short }}(r)$ and $\beta_{\text {mlow }}^{\text {long }}(r)$ measure the differential short and long term impacts in high minority areas within income quartiles 1 and 2 . $\beta_{\text {mhigh }}^{\text {short }}(r)$ and $\beta_{\text {mhigh }}^{\text {long }}(r)$ measure the differential short and long term impacts in high minority areas within income quartiles 3 and 4.

Figures 6 and 7 illustrate our nonparametric estimates in the short-term and long-term of the price gradient treatment effects given by equations (22) and (23) as well as $90 \%$ confidence intervals. Here, a negative estimate implies prices are increasing as one moves closer to the construction site, while a positive estimate implies prices are decreasing as one moves closer. Note that the results reported in the Q1/Q2 High Minority and Q3/Q4 High Minority plots are differential effects relative to the low minority effects reported in the Q1-Q4 plots. The Q1/Q2 High Minority plot illustrates that there may be slightly larger price appreciation 
effects in low income areas when the area is also high minority, however the effect is not quite statistically significant. Further, the statistically significant negative impact on the price gradient in the Q3/Q4 High Minority plot demonstrates that high income, high minority areas suffer significantly less price depreciation than high income, low minority areas. This is, of course, consistent with the evidence see in the second panel of Figure 5. High minority and low income areas receive the most house price appreciation from LIHTC development, while higher income, low minority areas exhibit house price decline.

\subsection{Mechanisms}

Taken together, these results seem to imply that LIHTC construction makes low income neighborhoods more desirable regardless of minority share, while making high income, low minority share neighborhoods less desirable. There are a variety of possible explanations for this finding. Even if LIHTC development is the initial shock that causes these house price changes, there are likely many indirect mechanisms through which LIHTC impacts the desirability of the local neighborhood. We begin by using the merged DataQuick-HMDA data and our nonparametric methods to investigate the impact of LIHTC development on local demographic change. If local residents have preferences over the demographics of their neighbors, the in-migration of LIHTC residents may further attract different types of residents and these new in-migrants could make the neighborhood more or less desirable. Figure 8 reports the average treatment effect in levels (not gradients) from years 0 to 10 on home buyer income. Consistent with our price results, we find that the introduction of affordable housing leads to home buyers with higher incomes of approximately $3 \%-4 \%$ in low income, low minority-share areas. Conversely, such introduction leads to a statistically significant decrease in home buyer income of approximately $1.5 \%$ in low minority, top income quartile areas. The effects are muted in high minority areas, with low-income high minority areas not attracting quite as high income home buyers as the low-income non-minority areas. The high income, high minority areas also do not experience declines in home buyer income, unlike the high income, low minority areas.

We next investigate the impact of LIHTC construction on the Black share of home buyers, with the results presented in Figure 9. The average impact on low income, low minority share areas is statistically and economically insignificant. However, low-income, high-minority areas do see a statistically significant decrease in the percentage of Black home buyers. We also see decreases in the Black share of home buyers in the higher income, high minority areas of 3 percentage points. Therefore, it appears that building affordable housing in high minority areas may lead to lower racial segregation. 
We finally explore the impact of affordable housing development on local crime rates in Figure 10. The cities of Chicago, San Francisco, and San Diego provide comprehensive detailed local crime statistics dating from 2001-2014 in Chicago, 2003-2014 in San Diego, and 2007-2014 in San Francisco. These data provide the type of crime, as well as the date and the exact location in the city. Since this is a much smaller sample (we have only have 127 LIHTC sites developed in this time frame in these cities), we cut the data only by high/low income and high/low minority. We find both violent and property crime decline in low income areas, regardless of minority share. However, in higher income areas we do not see any increase in crime, rather property crime may even fall slightly. Lowering crime in low income areas appears to be one of the driving mechanisms through which LIHTC improves low income neighborhoods. ${ }^{28}$

\subsection{Model Estimates}

To keep the model parsimonious, we only use the short term and long term price gradient estimates to recover households' preferences for LIHTC proximity. We denote these $\frac{\partial \widetilde{m}_{\mathbf{Y}}^{\text {short }}\left(r_{j}\right)}{\partial r}$ and $\frac{\partial \widetilde{m}_{\mathbf{Y}}^{\text {long }}\left(r_{j}\right)}{\partial r}$, as in equations $(22)$ and $(23)$.

Figure 6 plots the estimates of the short-term price gradient effects and Figure 7 plots the estimates of the long-term price gradient effects. These are labeled the "non-parametric estimates" in the figures. To use these estimates within our structural model to recover preferences, we set the gradient equal to zero at all distances past the point where the gradient first hits the $\mathrm{x}$-axis and crosses zero or the point at which it comes the closest to zero. These estimates are also shown in Figures 8 and $9 .{ }^{29}$ We use the point at which the price effect goes to zero as our estimate of $R_{0, \mathbf{Y}}$, the distance beyond which household utility is no longer impacted by LIHTC proximity.

Using the estimated price gradients, we use equations (5) and (4) to estimate each household's preference to live near LIHTC within neighborhood type $\mathbf{Y}, \hat{\gamma}_{i, \mathbf{Y}}:{ }^{30}$

$$
\begin{aligned}
& \text { if } R^{*}<R_{0, \mathbf{Y}} \text { and } \frac{\partial \widetilde{m}_{\mathbf{Y}}^{\tau_{s}}\left(r_{j}\right)}{\partial r}>0: \hat{\gamma}_{i, \mathbf{Y}}=\frac{\partial \widetilde{m}_{\mathbf{Y}}^{\tau_{s}}\left(r_{j}\right)}{\partial r}\left(1+R_{i}^{*}\right), \\
& \text { if } R^{*}<R_{0, \mathbf{Y}} \text { and } \frac{\partial \widetilde{m}_{\mathbf{Y}}^{\tau_{s}}\left(r_{j}\right)}{\partial r}<0: \hat{\gamma}_{i, \mathbf{Y}}=-\frac{\partial \widetilde{m}_{\mathbf{Y}}^{\tau_{s}}\left(r_{j}\right)}{\partial r}\left(1+R_{0, \mathbf{Y}}-R_{i}^{*}\right)
\end{aligned}
$$

\footnotetext{
${ }^{28}$ It is not uncommon for LIHTC developments to include live-in 24 hour security guards. Local police sometimes also set up an outpost within LIHTC developments. These investments may be part of the reason crime declines.

${ }^{29}$ Our raw estimates are never statistically different from zero at any distance past the point where gradient first hits the x-axis.

${ }^{30}$ We identify each household's preference for LIHTC proximity within neighborhood of type $\mathbf{Y}$ that the household chose to live in.
} 
where $\tau_{s}$ represents whether the household purchased their home in the short term or long term after LIHTC development. We designate LIHTC proximity to be desirable within neighborhoods where LIHTC development caused price appreciation, while we designate LIHTC proximity as undesirable within areas where it causes price decline.

We now examine how households' preferences for LIHTC proximity vary with race and income of home buyers. We measure how much each household is willing to pay (as a share of their house price) to live 0.1 miles from an LIHTC development. Panel A of Figures 11 and 12 shows that higher income households are willing to pay the most to live close the LIHTC development in Q1 income areas, conditional on wanting to live in a Q1 income area. This is true in both high and low minority areas. We find minority home buyers are willing to pay more to live in high minority, Q1 income areas than non-minority home buyers, while the reverse is true in low minority Q1 income areas. Overall, households choosing to live near LIHTC site in Q1 income areas are willing to pay about $6 \%$ of their house price to live 0.1 miles from an LIHTC site. The preferences in Q2 areas are essentially zero, as reflected in the essentially zero price effects discussed previously. Within low minority Q3 areas and Q4 areas, we find that higher income households are willing to pay slightly more to avoid living 0.1 miles from an LIHTC site. We also find that minority home buyers are less deterred by LIHTC development in Q3 and Q4 low minority areas than non-minority home buyers. Overall, these households are willing to pay about $1.6 \%$ of their house price to avoid living within 0.1 miles of LIHTC. Within Q3 and Q4 high minority areas, the effects are economically insignificant, consistent with the economically insignificant price effects discussed previously. Appendix figure A1 and A2 report similar effects measured in dollars, instead of in house price percentages, as these will be the numbers more closely linked to those used in the next section for the welfare calculation. These figures are dominated by the fact that higher income households buy more expensive houses, creating a strong link between home buyer income and willingness to pay for LIHTC proximity.

\section{Welfare}

We consider the local, long term welfare impact of building affordable housing in a variety of neighborhood types $\mathbf{Y} \in \mathcal{L}_{\mathbf{Y}}$, holding fixed the desirability of all other neighborhoods outside of the LIHTC area. ${ }^{31}$ In the pre-period, prior to construction of a LIHTC development, the local population optimizes over a vector of housing and neighborhood characteristics $(\mathbf{X}, \boldsymbol{\xi})$

\footnotetext{
${ }^{31}$ While we are able to quantify the welfare benefits of LIHTC development locally, we cannot account for potential broader impacts on the city/state as a whole. For example, if LIHTC simply relocated crime to other neighborhoods in a dispersed manner, we cannot capure the welfare effects of the dispersed impacts across the whole city.
} 
according to the following problem:

$$
\max u_{i}(\mathbf{X}, \boldsymbol{\xi})+c \quad \text { s.t. } \quad h_{0}(\mathbf{X}, \boldsymbol{\xi})+c \leq y_{i}
$$

where $u_{i}(\mathbf{X}, \boldsymbol{\xi})$ is an individual-specific utility function and $h_{0}(\mathbf{X}, \boldsymbol{\xi})$ is the equilibrium hedonic price function over characteristics in the pre-period. ${ }^{32}$ We denote the optimal bundle of characteristics chosen by household $i$ prior to LIHTC construction as $\left(\mathbf{X}_{0, i}^{*}, \boldsymbol{\xi}_{0, i}^{*}\right)$. Let $R_{\text {pre }}$ denote the distance from the future LIHTC site at this optimal bundle of characteristics. ${ }^{33}$

We assume zero moving costs, such that when affordable housing is built, all individuals in the local population will reoptimize according to the problem:

$$
\max _{R, X, \boldsymbol{\xi}, c} U_{i}(R, \mathbf{X}, \mathbf{Y}, \boldsymbol{\xi}, c) \quad \text { such that } \quad p_{1}(R, \mathbf{X}, \boldsymbol{\xi})+c \leq y_{i}+p_{1}\left(R_{p r e}, \mathbf{X}_{0, i}^{*}, \boldsymbol{\xi}_{0, i}^{*}\right)
$$

where the individual utility function follows the parametric form defined in equations (4) and (5). Here,

$$
p_{1}(R, \mathbf{X}, \boldsymbol{\xi})=\widetilde{m}_{\mathbf{Y}}(R)+h_{1}(\mathbf{X}, \boldsymbol{\xi})
$$

is the new equilibrium hedonic price function, with $\widetilde{m}_{\mathbf{Y}}(R)=0$ for all $R>R_{0, \mathbf{Y}}$ The quantity $p_{1}\left(R_{\text {pre }}, \mathbf{X}_{0, i}^{*}, \boldsymbol{\xi}_{0, i}^{*}\right)$ in the budget constraint is the revenue received from the sale of the current home. We denote optimal choices in the post period by agent $i$ as $\left(R_{1, i}^{*}, \mathbf{X}_{1, i}^{*}, \boldsymbol{\xi}_{1, i}^{*}, c_{1, i}^{*}\right)$. We assume that $h_{1}(\mathbf{X}, \boldsymbol{\xi})=h_{0}(\mathbf{X}, \boldsymbol{\xi})$ such that all agents choose the same bundle of house of characteristics $(\mathbf{X}, \boldsymbol{\xi})$ in the post-period as in the pre-period.

The welfare impacts of LIHTC construction accrues to homeowners, renters, and absentee landlords. For homeowners, if $R_{\text {pre }} \geq R_{0, \mathbf{Y}}$ and $R_{1, i}^{*} \geq R_{0, \mathbf{Y}}$, then the welfare impact $\Delta U_{i}=0$. The effect of LIHTC on utility is zero at distances beyond $R_{0, \mathbf{Y}}$, agents do not choose an alternative bundle of house characteristics $(\mathbf{X}, \boldsymbol{\xi})$, as they can obtain their original bundle of house characteristics for the same price. For homeowners who live at a location $R_{\text {pre }}<R_{0, \mathbf{Y}}$ in the pre-period, but optimally choose $R_{1, i}^{*} \geq R_{0, \mathbf{Y}}$ post construction, the welfare impact is given by the change in prices:

$$
\Delta U_{i}=\widetilde{m}_{\mathbf{Y}}\left(R_{p r e}\right)
$$

These households move away from the LIHTC development, but face welfare impacts from the amount of house price appreciation or depreciation $\left(\widetilde{m}_{\mathbf{Y}}\left(R_{\text {pre }}\right)\right)$ they must realize in

\footnotetext{
${ }^{32}$ We assume that agents do not anticipate LIHTC development when they choose their optimal bundle of characteristics in the pre-period.

${ }^{33}$ Note that $R_{\text {pre }}$ is not chosen by agents in the pre-period, but is a function of the phsyical location of the optimal bundle $\left(\mathbf{X}_{0, i}^{*}, \boldsymbol{\xi}_{0, i}^{*}\right)$.
} 
order to move.

For homeowners who optimally choose $R_{1, i}^{*}<R_{0, \mathbf{Y}}$, the welfare impact is given by the resulting utility gain and change in prices. For areas where being closer to LIHTC is desirable we have:

$$
\begin{array}{rlrl}
\Delta U_{i} & =\gamma_{i, \mathbf{Y}} \log \left(1+R_{0, \mathbf{Y}}-R_{1, i}^{*}\right)+\tilde{m}_{\mathbf{Y}}\left(R_{\text {pre }}\right)-\tilde{m}_{\mathbf{Y}}\left(R_{1, i}^{*}\right), & \text { if } R_{\text {pre }} \leq R_{0, \mathbf{Y}} \\
\Delta U_{i}=\gamma_{i, \mathbf{Y}} \log \left(1+R_{0, \mathbf{Y}}-R_{1, i}^{*}\right)-\widetilde{m}_{\mathbf{Y}}\left(R_{1, i}^{*}\right), & \text { if } R_{\text {pre }}>R_{0, \mathbf{Y}} .
\end{array}
$$

For areas where being further from LIHTC is desirable we have:

$$
\begin{aligned}
\Delta U_{i}=\gamma_{i, \mathbf{Y}} \log \left(\frac{1+R_{1, i}^{*}}{1+R_{0, \mathbf{Y}}}\right)+\tilde{m}_{\mathbf{Y}}\left(R_{p r e}\right)-\widetilde{m}_{\mathbf{Y}}\left(R_{1, i}^{*}\right), & \text { if } R_{\text {pre }} \leq R_{0, \mathbf{Y}} \\
\Delta U_{i}=\gamma_{i, \mathbf{Y}} \log \left(\frac{1+R_{1, i}^{*}}{1+R_{0, \mathbf{Y}}}\right)-\widetilde{m}_{\mathbf{Y}}\left(R_{1, i}^{*}\right), & \text { if } R_{\text {pre }}>R_{0, \mathbf{Y}} .
\end{aligned}
$$

Since we do not observe data directly on renters, we must make the assumption that house prices are equal to the present discounted value of rents. The renter's utility optimization is:

$$
\max _{R, X, \boldsymbol{\xi}, c} U_{i}(R, \mathbf{X}, \mathbf{Y}, \boldsymbol{\xi}, c) \text { such that } p_{1}(R, \mathbf{X}, \boldsymbol{\xi})+c \leq y_{i} .
$$

The only difference between the renter's and home owner's optimization is that homeowners receive the revenue from the house sale of their previous residence. This money, for renters, would go to the landlord in the form of rent payments.

We find that the welfare impacts for renters who optimally choose $R_{1, i}^{*}<R_{0, \mathbf{Y}}$ in areas where being closer to LIHTC is desirable are given by:

$$
\Delta U_{i}=\gamma_{i, \mathbf{Y}} \log \left(1+R_{0, \mathbf{Y}}-R_{1, i}^{*}\right)-\widetilde{m}_{\mathbf{Y}}\left(R_{1, i}^{*}\right)
$$

Renters benefit from LIHTC proximity, but also face the negative impact of having to pay higher rents. Within areas where being further from LIHTC is desirable renters' utility is given by:

$$
\Delta U_{i}=\gamma_{i, \mathbf{Y}} \log \left(\frac{1+R_{1, i}^{*}}{1+R_{0, \mathbf{Y}}}\right)-\widetilde{m}_{\mathbf{Y}}\left(R_{1, i}^{*}\right)
$$

While these renters do not like living near LIHTC, they are compensated for it by the lower rents. On net, these renters are made strictly better off since they could have chosen to live far away from the LIHTC site and receive a utility of zero. The impact is zero for renters who 
optimally choose $R_{1, i}^{*}>R_{0, \mathbf{Y}} \cdot{ }^{34}$ Finally, the welfare impact on absentee landlords whose properties are located at $R_{1, i}^{*} \leq R_{0, \mathbf{Y}}$ is given by the present value of the change in rents they collect:

$$
\Delta U_{i}=\widetilde{m}_{\mathbf{Y}}\left(R_{1, i}^{*}\right)
$$

The impact is zero for landlords with properties located at distances greater than $R_{0, \mathbf{Y}}$ since rents do not change. These impacts are summarized in Tables 2 and 3.

We aggregate these individual welfare effects to calculate the total welfare impact on homeowners, renters, and absentee landlords. To this end, let $q^{H}\left(\gamma_{\mathbf{Y}}, R_{1}\right.$, Race, $\left.y \mid \mathbf{Y}, R_{1}<R_{0, \mathbf{Y}}\right)$ denote the joint density of preference parameters, distance chosen from the LIHTC site, race, and income given that the household has chosen to live in a neighborhood of type $\mathbf{Y}$, with $R_{1}<R_{0, \mathbf{Y}}$ miles from an LIHTC site. This density can be directly estimated from the merged DataQuick-HMDA data. Then the aggregate welfare impact of a LIHTC site developed in neighborhood of type $\mathbf{Y}$ for homeowners is given by (for each amenity/disamenity type):

$$
\begin{aligned}
\Delta U_{a g g, \mathbf{Y}}^{H} & =N_{H} \sum_{\text {Race }} \int \gamma_{\mathbf{Y}} \log \left(1+R_{0, \mathbf{Y}}-R_{1, i}^{*}\right) q^{H}\left(\gamma_{\mathbf{Y}}, R_{1}, \text { Race, } y \mid \mathbf{Y}, R_{1}<R_{0, \mathbf{Y}}\right) d \gamma_{\mathbf{Y}} d R_{1} d y \\
\Delta U_{a g g, \mathbf{Y}}^{H} & =N_{H} \sum_{\text {Race }} \int \gamma_{\mathbf{Y}} \log \left(\frac{1+R_{1, i}^{*}}{1+R_{0, \mathbf{Y}}}\right) q^{H}\left(\gamma_{\mathbf{Y}}, R_{1}, \text { Race, } y \mid \mathbf{Y}, R_{1}<R_{0, \mathbf{Y}}\right) d \gamma_{\mathbf{Y}} d R_{1} d y
\end{aligned}
$$

where $N_{H}$ is the average number of homeowners who choose to live around a single LIHTC site in neighborhoods of type $\mathbf{Y}$, within $R_{0, \mathbf{Y}}$ miles of the LIHTC site. We measure $N_{H}$ from the 5-year pooled American Community Survey block group data on the median number of homeowners per LIHTC site living within $R_{0, \mathbf{Y}}$ miles of the LIHTC site of type $\mathbf{Y}{ }^{35}$ These numbers are reported in Table A1.

To calculate the impact for renters we assume that the density of preference parameters and chosen LIHTC distances conditional on race and income is the same as that for homeowners, that is:

$$
q^{R}\left(\gamma_{\mathbf{Y}}, R_{1} \mid \text { Race, } y, \mathbf{Y}, R_{1}<R_{0, \mathbf{Y}}\right)=q^{H}\left(\gamma_{\mathbf{Y}}, R_{1} \mid \text { Race, } y, \mathbf{Y}, R_{1}<R_{0, \mathbf{Y}}\right)
$$

We can then calculate the aggregate welfare impact on renters as (within areas where LIHTC

\footnotetext{
${ }^{34}$ This places a lower bound on the welfare impact on each household. For some households, the predicted welfare effects appear below this bound because the functional form of the utility function is not curved enough to satisfy the lower bound on renter utility. We truncate the welfare effects for these household to be equal to the lower bound of zero welfare effects.

${ }^{35}$ We meaure these household counts for the LIHTC sites used in our estimation, not the entire country.
} 
is viewed as an amenity):

$$
\begin{aligned}
\Delta U_{a g g, \mathbf{Y}}^{R} & =N_{R} \sum_{\text {Race }} \int\left[\gamma_{\mathbf{Y}} \log \left(1+R_{0, \mathbf{Y}}-R_{1, i}^{*}\right)-\widetilde{m}_{\mathbf{Y}}\left(R_{1}\right)\right] q^{R}\left(\gamma_{\mathbf{Y}}, R_{1}, \text { Race, } y\right) d \gamma_{\mathbf{Y}} d R_{1} d y \\
& =N_{R} \sum_{\text {Race }} \int\left[\gamma_{\mathbf{Y}} \log \left(1+R_{0, \mathbf{Y}}-R_{1, i}^{*}\right)-\widetilde{m}_{\mathbf{Y}}\left(R_{1}\right)\right] q^{R}\left(\gamma_{\mathbf{Y}}, R_{1} \mid \text { Race, } y\right) q^{R}\left(\text { Race, y)d } \gamma_{\mathbf{Y}} d R_{1} d y\right. \\
& =N_{R} \sum_{\text {Race }} \int\left[\gamma_{\mathbf{Y}} \log \left(1+R_{0, \mathbf{Y}}-R_{1, i}^{*}\right)-\widetilde{m}_{\mathbf{Y}}\left(R_{1}\right)\right] q^{H}\left(\gamma_{\mathbf{Y}}, R_{1} \mid \text { Race, } y\right) q^{R}(\text { Race, } y) d \gamma_{\mathbf{Y}} d R_{1} d y \\
& =N_{R} \sum_{\text {Race }} \int\left[\gamma_{\mathbf{Y}} \log \left(1+R_{0, \mathbf{Y}}-R_{1, i}^{*}\right)-\widetilde{m}_{\mathbf{Y}}\left(R_{1}\right)\right] q^{H}\left(\gamma_{\mathbf{Y}}, R_{1}, \text { Race, } y\right) \frac{q^{R}(\text { Race }, y)}{q^{H}(\text { Race }, y)} d \gamma_{\mathbf{Y}} d R_{1} d y
\end{aligned}
$$

In areas where LIHTC is viewed as a disamenity we have:

$$
\Delta U_{a g g, \mathbf{Y}}^{R}=N_{R} \sum_{\text {Race }} \int\left[\gamma_{\mathbf{Y}} \log \left(\frac{1+R_{1, i}^{*}}{1+R_{0, \mathbf{Y}}}\right)-\widetilde{m}_{\mathbf{Y}}\left(R_{1}\right)\right] q^{H}\left(\gamma_{\mathbf{Y}}, R_{1}, \text { Race, } y\right) \frac{q^{R}(\text { Race }, y)}{q^{H}(\text { Race }, y)} d \gamma_{\mathbf{Y}} d R_{1} d y
$$

The joint density of race and income for both renters and homeowners, $q^{R}($ Race, $y)$ and $q^{H}($ Race,$y)$ respectively, are calculated from 5-year pooled American Community Survey micro data. For the price effects due to LIHTC proximity, $\widetilde{m}_{\mathbf{Y}}$, we will use our long term price estimates, derived from equation 23. Finally, the aggregate impact on absentee landlords is given by:

$$
\Delta U_{a g g}^{l l}=N_{R} \sum_{\text {Race }} \int \widetilde{m}_{\mathbf{Y}}\left(R_{1}\right) q^{H}\left(\gamma_{\mathbf{Y}}, R_{1}, \text { Race, } y\right) \frac{q^{R}(\text { Race }, y)}{q^{H}(\text { Race }, y)} d \gamma_{\mathbf{Y}} d R_{1} d y
$$

Panel A of Table 4 reports the average willingness to pay for LIHTC development per homeowner, renter, and landlord impacted by LIHTC development within neighborhoods of different types. Within Q1 income, low minority areas, the average homeowner would be willing to pay $\$ 23,403$ for LIHTC development. The average renter would be willing to pay $\$ 6502$ and the average landlord would be willing to pay $\$ 6011 .^{36}$ In Q1, high minority areas, the average homeowner would be willing to pay $\$ 16,857$, the average renter would be willing to pay $\$ 6475$, and the average landlord would be willing to pay $\$ 6099$. There are substantial benefits to the community from LIHTC development in Q1 income areas. However, the opposite is true in low minority Q3 and Q4 areas. Within Q4 areas, the average homeowner would be willing to pay $\$ 3972$ to deter LIHTC development, the average renter would be willing to be $\$ 67$ for LIHTC development and the average landlord would be willing to pay

\footnotetext{
${ }^{36}$ The sum of the welfare benefits to landlords and renters is less than that of homeowners because renters tend to be lower income, lowering their willingness to pay.
} 
$\$ 2416$ to deter LIHTC development. In high minority, Q4 income areas, residents benefit from LIHTC development.

Table 5 scales these numbers to aggregate effects to get total willingness to pay by society from LIHTC development in different areas. The units are reported in thousands of dollars. Since low income areas tend to be quite dense, as evidenced by Table A1, the aggregate benefit to homeowners from LIHTC development in Q1 income low minority areas is $\$ 57.9$ million. The aggregate benefit to renters is $\$ 29.2$ million and the benefit to landlords is $\$ 29.0$ million. In total, society would be improved by $\$ 116.2$ million from a single LIHTC development in a low income, low minority area. This number is even bigger in low income, high minority areas, (\$211 million), simply because there tend to be more people living close to LIHTC in these areas. Conversely, development of LIHTC in a Q4, low minority area leads to an aggregate welfare loss of $\$ 12.1$ million.

Viewing LIHTC development as a place based policy, these estimates suggests that there could be large welfare gains from development to low income areas. However, the location of affordable housing also influences the welfare of the tenants living in the affordable housing. Recent work by Chetty et al. (2015) finds that young children strongly benefit from growing up in lower poverty neighborhoods. While the neighborhoods types analyzed in Chetty et al. (2015) do not map directly into our definitions, they report that a child moving out of public housing and into a low poverty area gains $\$ 99,000$ in presented discounted value of future income over a lifetime. If we use this number to benchmark the potential gains to LIHTC tenants living in a Q4 low minority area versus a Q1 low minority neighborhood, and assume each apartment has two children in it, the average LIHTC development would improve the welfare of these children by $\$ 26.7$ million. ${ }^{37}$ This is an under estimate of the total welfare benefits to these households, as it only values the increased earnings and not other benefits which have been documented, such as better measures of mental health and lower obesity rates (Kling et al, 2007). The benefits to these tenants (\$26.7 million) more than offsets the losses to local residents in these high income areas (\$12.1 million). However, there is an opportunity cost of $\$ 116$ million by not developing and investing in the lowest income neighborhoods, which is more than 4 times the welfare benefits documented by Chetty et al. (2015). This makes development of affordable housing in low income areas appear to improve the economic welfare of many low-income households. We must caveat these points with the fact that there may likely be diminishing marginal returns to new housing in poor

\footnotetext{
${ }^{37}$ We assume the LIHTC remains affordable for 30 years and that each apartment will house a household with two children for 15 years. Thus, two households will leave in each apartment for 15 years, sequentiailly. We discount the present value of childrens' income of the 2nd household moving in the apartment 15 years after it was built by 3\%, same discount rate used in Chety et al. (2015). We assume the LIHTC site at 82 apartments.
} 
areas if these policies were scaled substantially, however this is likely also true of the benefits estimated by Chetty et al (2015).

\section{Conclusion}

In this paper, we study multifamily housing developments funded through the Low Income Housing Tax Credit (LIHTC) to quantify the costs and benefits of affordable housing development on surrounding neighborhoods. Leveraging new econometric methods, we find that LIHTC construction has heterogenous effects on local house prices based on neighborhood characteristics. In lower income areas, house prices appreciate substantially over the long-run in response to the introduction of affordable housing projects. Areas with a high minority share also experience significant price appreciation when a LIHTC development is built. On the other hand, prices in areas with higher median incomes and low minority shares tend to depreciate over the long-run.

We employ a structural, generalized hedonic model of housing choice to link these estimated price effects to individual preferences for proximity to low income housing. In areas where affordable housing developments are viewed as an amenity, higher income households are willing to pay more for proximity. Conversely, higher income households are willing to pay more to live further away from affordable housing developments in areas where such properties are viewed as a disamenity.

Our results show that affordable housing development has large welfare impacts as a place based policy, which more than offset the welfare impacts to tenants living in affordable housing. Given the goals of many affordable housing policies is to decrease income and racial segregation in housing markets, these goals might be achieved by investing in affordable housing in low income and high minority areas, which will then spark in-migration of high income and a more racially diverse set of residents. 


\section{References}

Aliprantis, D. (2014). "Assessing the Evidence on Neighborhood Effects from Moving to Opportunity," Federal Reserve Bank of Cleveland working paper 10-33R.

Aliprantis, D. and D. Hartley (2014). "Blowing It Up and Knocking It Down: The Local and Citywide Effects of Demolishing High-Concentration Public Housing on Crime," Federal Reserve Bank of Cleveland working paper 10-22R.

Autor, D., Palmer, C. J., and P. A. Pathak. (2014). "Housing Market Spillovers: Evidence from the End of Rent Control in Cambridge, Massachusetts," Journal of Political Economy 122(3): 661-717.

Bajari, P. and C. L. Benkard (2005). "Demand Estimation with Heterogeneous Consumers and Unobserved Product Characteristics: A Hedonic Approach," Journal of Political Economy 113(6): 1239-1276.

Baum-Snow, N. and J. Marion (2009). "The Effects of Low Income Housing Tax Credit Developments on Neighborhoods," Journal of Public Economics 93(5-6): 654-666.

Bayer, P., F. Ferreira and R. McMillian (2007). "A Unified Framework for Measuring Preferences for Schools and Neighborhoods," Journal of Political Economy 115(4): 588-638.

Berry, S., J. Levinsohn and A. Pakes (1995). "Automobile Prices in Market Equilibrium," Econometrica 63(4). 841-890.

Berry, S., \& Pakes, A. (2007). "The pure characteristics demand model," International Economic Review, 48(4), 1193-1225.

Campbell, J. Y., Giglio, S., \& Pathak, P. (2011). Forced Sales and House Prices. The American Economic Review, 2108-2131.

Card, D., Mas, A., \& Rothstein, J. (2008). Tipping and the Dynamics of Segregation. The Quarterly Journal of Economics, 177-218.

Charnigo, R., Hall, B., Srinivasan, C. (2011). "A Generalized Cp Criterion for Derivative Estimation." Technometrics, 53, 238-253.

Charnigo, R., and C. Srinivasan (2015). "A Multivariate Generalized $C_{p}$ and Surface Estimation," Biostatistics, 16, 311-325.

Chetty, R., N. Hendren, and L. Katx (2015) "The Effects of Exposure to Better Neighborhoods on Children: New Evidence from the Moving to Opportunity Experiment" Harvard University working paper.

Diamond, R. (2015). "The Determinants and Welfare Implications of US Workers' Diverging Location Choices by Skill: 1980-2000," American Economic Review, forthcoming.

Ellen, I. G., Lacoe, J., \& Sharygin, C. A. (2013). Do foreclosures cause crime?. Journal of Urban Economics, 74, 59-70.

Goujard, A. (2011). "The spillovers from social housing, evidence from housing prices," LSE working paper. 
Erikesen, M. (2009). "The market price of Low-Income Housing Tax Credits," Journal of Urban Economics 66: 141-149.

Erikesen, M. and S. Rosenthal (2010). "Crowd out effects of place-based subsidized rental housing: New evidence from the LIHTC program," Journal of Public Economics 94: 953-966.

Green, R., S. Malpezzi and K. Seah (2002). "Low Income Housing Tax Credit Housing Developments and Property Values," The Center for Urban Land Economics Research, University of Wisconsin

Guerrieri, V., E. Hurst and D. Hartley (2013). "Endogenous Gentrification and Housing Price Dynamics.," Journal of Public Economics 100: 45-60.

Handbury, J. (2013). "Are Poor Cities Cheap for Everyone? Non-Homotheticity and the Cost of Living Across U.S. Cities," University of Pennsylvania working paper.

Kling, J., J. Liebman and L. Katz (2007). "Experimental Analysis of Neighborhood Effects," Econometrica 75(1): 83-119.

McFadden, D. (1973). "Conditional Logit Analysis of Qualitative Choice Behavior," in Frontiers of Econometrics, ed. by P. Zarembka. New York: Academic Press.

Owens, R., E. Rossi-Hansberg and P. Sarte (2010). "Housing Externalities," Journal of Political Economy 118(3): 409-432.

Rosen, S. (1974). "Hedonic Prices and Implicit Markets: Product Differentiation in Pure Competition," Journal of Political Economy 82: 34-55.

Rossi-Hansberg, E., Sarte, P. D., \& Owens III, R. (2010). Housing Externalities. Journal of Political Economy, 118(3).

Schwartz, A., I. Ellen, I. Voicu and M. Schill (2006). "The External Effects of Subsidized Housing Investment," Regional Science and Urban Economics 36(2): 697-707.

Sinai, T. and J. Waldfogel (2005). "Do Low-Income Housing Subsidies Increase the Occupied Housing Stock?" Journal of Public Economics 89(11): 2137-2164. 
Table 1: Summary Statistics

Panel A: 1990 Census Block Group Data

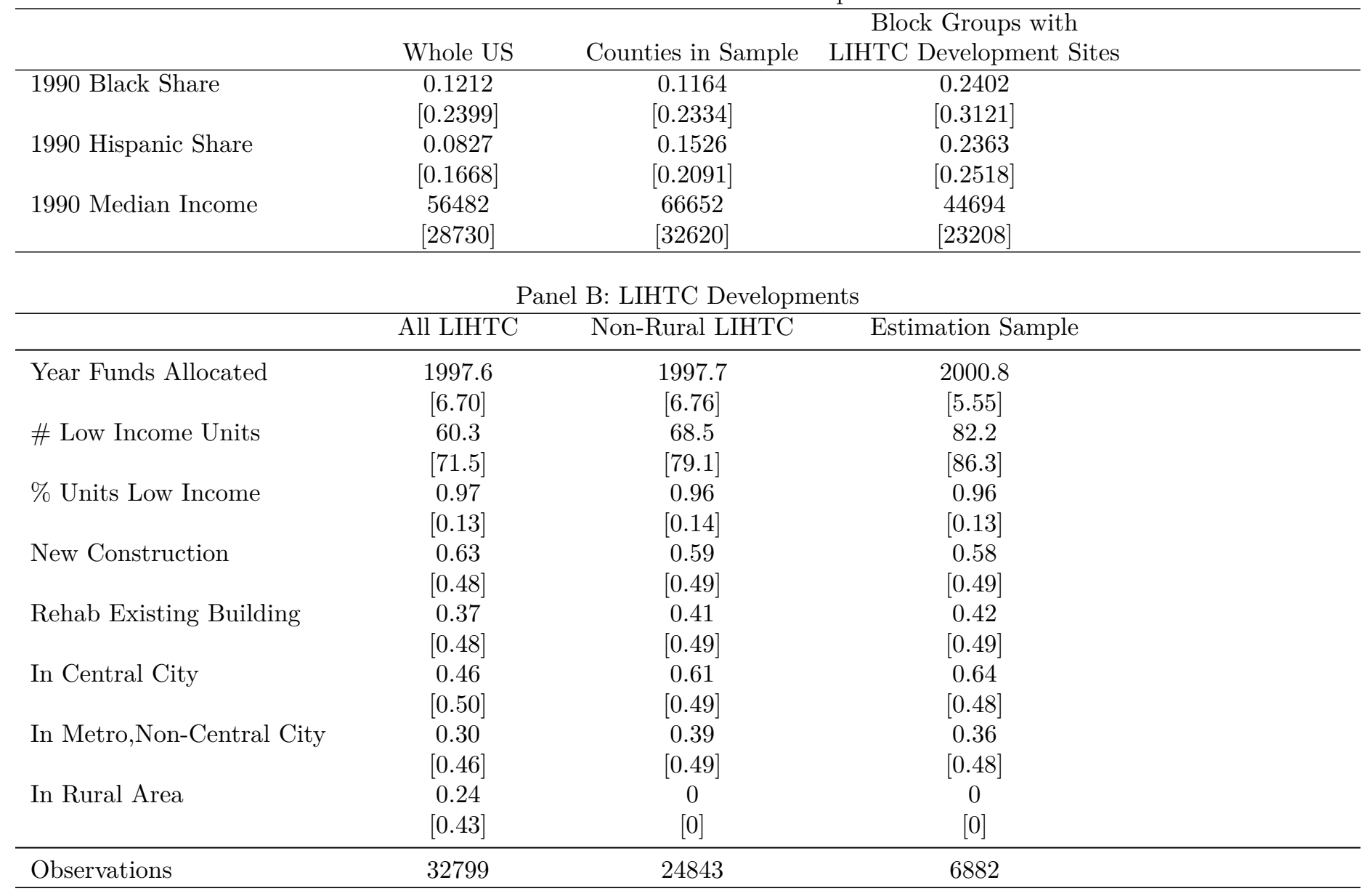

Panel C: DataQuick \& HMDA Data

\begin{tabular}{|c|c|c|c|c|}
\hline & $\begin{array}{c}\text { Transactions }<1 \\
\text { mi of LIHTC Site }\end{array}$ & $\begin{array}{l}\text { Transactions }<.5 \\
\text { mi of LIHTC Site }\end{array}$ & $\begin{array}{c}\text { Transactions }<.2 \\
\text { mi of LIHTC Site }\end{array}$ & $\begin{array}{c}\text { Transactions }<.1 \\
\text { mi of LIHTC Site }\end{array}$ \\
\hline Housing Transaction Price & $\begin{array}{c}305610 \\
{[336428]}\end{array}$ & $\begin{array}{c}284742 \\
{[380557]}\end{array}$ & $\begin{array}{c}268217 \\
{[404520]}\end{array}$ & $\begin{array}{c}270652 \\
{[471396]}\end{array}$ \\
\hline Housing Transaction Price & 323703 & 302575 & 284829 & 284256 \\
\hline -HMDA Matched & {$[319851]$} & [364203] & [377024] & [411580] \\
\hline$\%$ of Home Buyers Black & $\begin{array}{c}0.0612 \\
{[0.4670]}\end{array}$ & $\begin{array}{c}0.068 \\
{[0.4737]}\end{array}$ & $\begin{array}{c}0.0735 \\
{[0.4800]}\end{array}$ & $\begin{array}{c}0.0726 \\
{[0.4802]}\end{array}$ \\
\hline Income of Home Buyer & $\begin{array}{l}97619 \\
{[50859]}\end{array}$ & $\begin{array}{l}94375 \\
{[51099]}\end{array}$ & $\begin{array}{l}92649 \\
{[52625]}\end{array}$ & $\begin{array}{l}93299 \\
{[54365]}\end{array}$ \\
\hline Housing Transactions & 8164281 & 3430606 & 807411 & 241875 \\
\hline
\end{tabular}

Panel D: Crime Rates within $1 \mathrm{mi}$ of LIHTC Sites

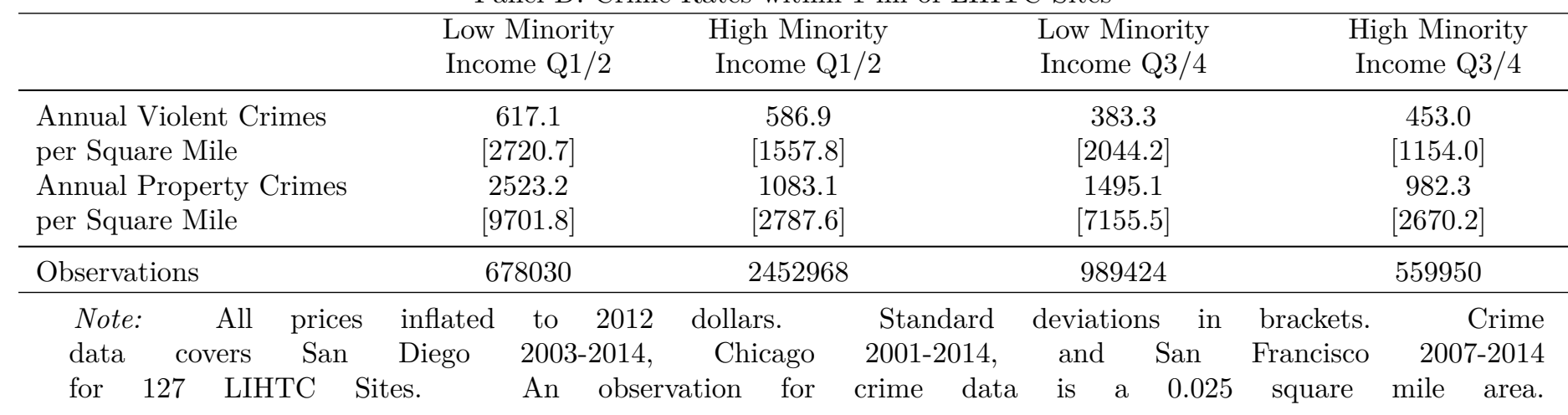


Table 2: Welfare Impacts of LIHTC on Households (LIHTC Amenity)

\begin{tabular}{|c|c|c|c|}
\hline$\Delta U_{i}$ & Homeowners & Renters & Landlords \\
\hline$R_{\text {pre }}>R_{0, \mathbf{Y}}, R_{1, i}^{*}>R_{0, \mathbf{Y}}$ & 0 & 0 & $\overline{0}$ \\
\hline$R_{\text {pre }}>R_{0, \mathbf{Y}}, R_{1, i}^{*}<R_{0, \mathbf{Y}}$ & $\gamma_{i, \mathbf{Y}} \log \left(1+R_{0, \mathbf{Y}}-R_{1, i}^{*}\right)-\widetilde{m}_{\mathbf{Y}}\left(R_{1, i}^{*}\right)$ & $\gamma_{i, \mathbf{Y}} \log \left(1+R_{0, \mathbf{Y}}-R_{1, i}^{*}\right)-\widetilde{m}_{\mathbf{Y}}\left(R_{1, i}^{*}\right)$ & 0 \\
\hline$R_{\text {pre }}<R_{0, \mathbf{Y}}, R_{1, i}^{*}>R_{0, \mathbf{Y}}$ & $\widetilde{m}_{\mathbf{Y}}\left(R_{\text {pre }}\right)$ & 0 & $\widetilde{m}_{\mathbf{Y}}\left(R_{1, i}^{*}\right)$ \\
\hline$R_{\text {pre }}<R_{0, \mathbf{Y}}, R_{1, i}^{*}<R_{0, \mathbf{Y}}$ & $\begin{array}{l}\gamma_{i, \mathbf{Y}} \log \left(1+R_{0, \mathbf{Y}}-R_{1, i}^{*}\right)+\widetilde{m}_{\mathbf{Y}}\left(R_{\text {pre }}\right)- \\
\widetilde{m}_{\mathbf{Y}}\left(R_{1, i}^{*}\right)\end{array}$ & $\gamma_{i, \mathbf{Y}} \log \left(1+R_{0, \mathbf{Y}}-R_{1, i}^{*}\right)-\widetilde{m}_{\mathbf{Y}}\left(R_{1, i}^{*}\right)$ & $\widetilde{m}_{\mathbf{Y}}\left(R_{1, i}^{*}\right)$ \\
\hline
\end{tabular}

Note: $R_{\text {pre }}$ represents distance of household's chosen location before LIHTC development from new LIHTC development site. $R_{1, i}^{*}$ represents chosen distance from LIHTC development site when household reoptimizes location post LIHTC development. $R_{0, \mathbf{Y}}$ represents max distance at which LIHTC proximity can impact utility. Each row and column pair represents a different household type. Entry summarizes welfare impact of LIHTC development when agents view LIHTC proximity as an amenity.

Table 3: Welfare Impacts of LIHTC on Households (LIHTC Disamenity)

\begin{tabular}{c|llr}
\hline$\Delta U_{i}$ & Homeowners & Renters & Landlords \\
\hline$R_{\text {pre }}>R_{0, \mathbf{Y}}, R_{1, i}^{*}>R_{0, \mathbf{Y}}$ & 0 & 0 & 0 \\
$R_{\text {pre }}>R_{0, \mathbf{Y}}, R_{1, i}^{*}<R_{0, \mathbf{Y}}$ & $\gamma_{i, \mathbf{Y}} \log \left(\frac{1+R_{1, i}^{*}}{1-R_{0, \mathbf{Y}}}\right)-\widetilde{m}_{\mathbf{Y}}\left(R_{1, i}^{*}\right)$ & $\gamma_{i, \mathbf{Y}} \log \left(\frac{1+R_{1, i}^{*}}{1-R_{0, \mathbf{Y}}}\right)-\widetilde{m}_{\mathbf{Y}}\left(R_{1, i}^{*}\right)$ & 0 \\
$R_{\text {pre }}<R_{0, \mathbf{Y}}, R_{1, i}^{*}>R_{0, \mathbf{Y}}$ & $\widetilde{m}_{\mathbf{Y}}\left(R_{\text {pre }}\right)$ & 0 & $\widetilde{m}_{\mathbf{Y}}\left(R_{1, i}^{*}\right)$ \\
$R_{\text {pre }}<R_{0, \mathbf{Y}}, R_{1, i}^{*}<R_{0, \mathbf{Y}}$ & $\gamma_{i, \mathbf{Y}} \log \left(\frac{1+R_{1, i}^{*}}{1-R_{0, \mathbf{Y}}}\right)+\widetilde{m}_{\mathbf{Y}}\left(R_{\text {pre }}\right)-\widetilde{m}_{\mathbf{Y}}\left(R_{1, i}^{*}\right)$ & $\gamma_{i, \mathbf{Y}} \log \left(\frac{1+R_{1, i}^{*}}{1-R_{0, \mathbf{Y}}}\right)-\widetilde{m}_{\mathbf{Y}}\left(R_{1, i}^{*}\right)$ & $\widetilde{m}_{\mathbf{Y}}\left(R_{1, i}^{*}\right)$ \\
\hline
\end{tabular}

Note: $R_{\text {pre }}$ represents distance of household's chosen location before LIHTC development from new LIHTC development site. $R_{1, i}^{*}$ represents chosen distance from LIHTC development site when household reoptimizes location post LIHTC development. $R_{0, \mathbf{Y}}$ represents max distance at which LIHTC proximity can impact utility. Each row and column pair represents a different household type. Entry summarizes welfare impact of LIHTC development when agents view LIHTC proximity as a disamenity. 
Table 4: Mean Utility Benefit per Household from LIHTC Construction

Panel A: Low Minority Areas

\begin{tabular}{lcccc}
\hline & Income Q1 & Income Q2 & Income Q3 & Income Q4 \\
\hline Average Benefit per Home Owner & 23403 & 208 & -3636 & -3972 \\
Average Benefit per Renter & 6502 & 67 & 234 & 67 \\
Average Benefit per Landlord & 6011 & 46 & -2843 & -2416 \\
\hline \multicolumn{2}{|c}{ Panel B: High Minority Areas } & & \\
\hline & Income Q1 & Income Q2 & Income Q3 & Income Q4 \\
\hline Average Benefit per Home Owner & 16857 & 2414 & 996 & 3255 \\
Average Benefit per Renter & 6475 & 190 & 342 & 971 \\
Average Benefit per Landlord & 6099 & 1288 & 375 & 1090 \\
\hline
\end{tabular}

Note: Mean welfare benefit from LIHTC construction to households who choose to live nearby. Utility is measured in 2012 dollars. To decompose effect between renters and landlords we assume the present discounted value of future rents is equal to house prices. 
Table 5: Total Utility Benefit to Households from LIHTC Construction

Panel A: Low Minority Areas

\begin{tabular}{lcccc}
\hline & Income Q1 & Income Q2 & Income Q3 & Income Q4 \\
\hline Aggregate Benefit to Home Owners & 57,945 & 61.46 & $-3,789$ & $-9,008$ \\
Aggregate Benefit to Renters & 29,208 & 23.87 & 258 & 78.48 \\
Aggregate Benefit to Landlords & 29,048 & 28.42 & $-3,331$ & $-3,208$ \\
Aggregate Benefit to Society & 116201 & 113.7 & $-6,861$ & $-12,138$ \\
\hline & Panel B: High Minority Areas & & Income Q4 \\
\hline & Income Q1 & Income Q2 & Income Q3 & 14,508 \\
& 63,460 & 1,446 & 3,615 & 4,861 \\
Aggregate Benefit to Home Owners & 73,417 & 314.7 & 1,903 & 5,907 \\
Aggregate Benefit to Renters & 74,236 & 2,672 & 2,262 & 25,277 \\
Aggregate Benefit to Landlords & 21113 & 4,433 & 7,780 & \\
Aggregate Benefit to Society & & &
\end{tabular}

Note: Total welfare benefit from LIHTC construction to households. Utility is measured in thousands of 2012 dollars. To decompose effect between renters and landlords we assume the present discounted value of future rents is equal to house prices. 
Figure 1: Counties Used in Analysis

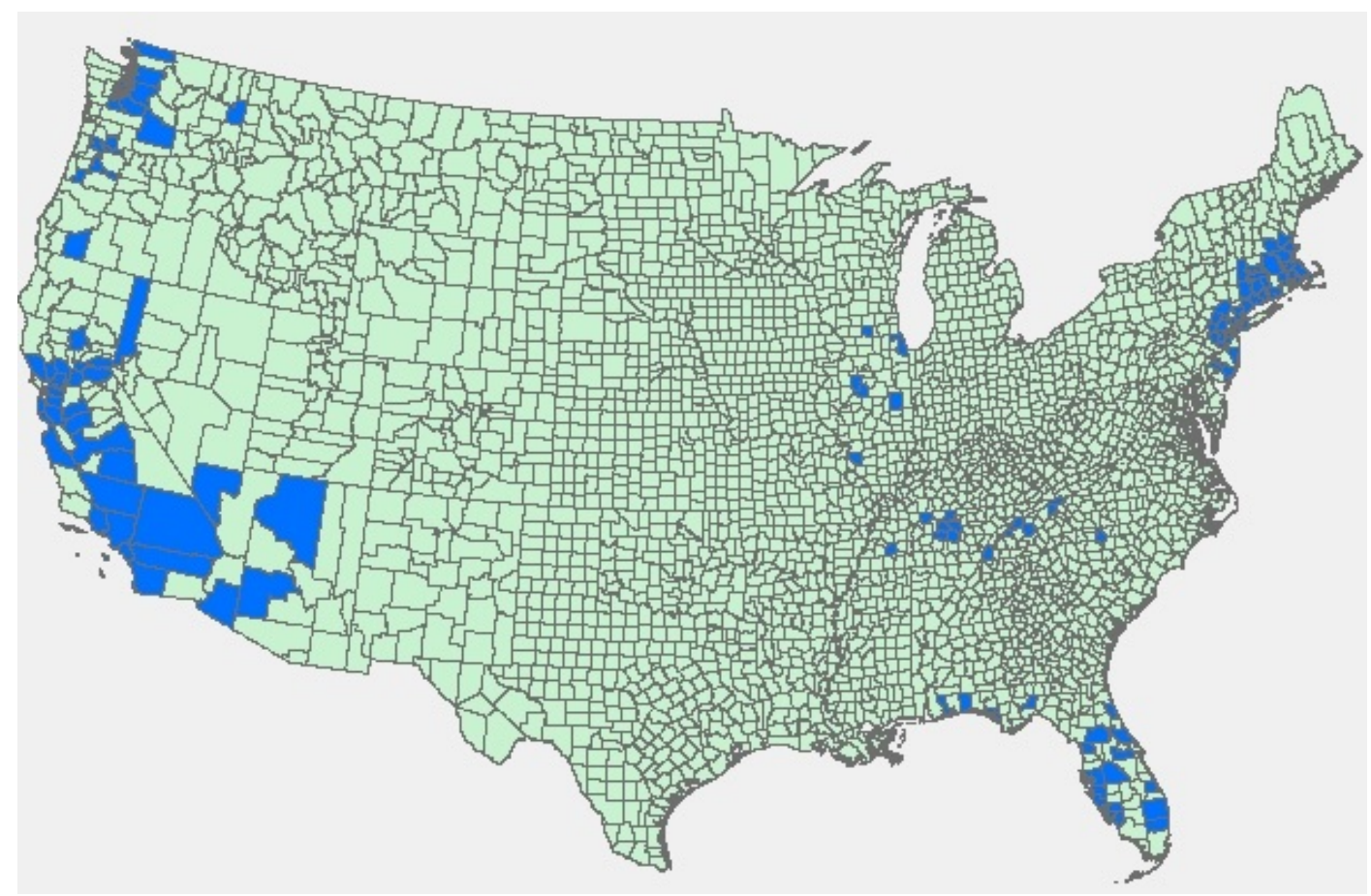

Note: Counties were selected based on whether the history of housing transaction data began in 1996 or earlier and had at least an average of 1000 arm-length transactions per year. This gives 129 counites in 15 states and covers $31 \%$ of the US population. 
Figure 2: Example of Bowtie Threshold Used in Empirical Derivatives

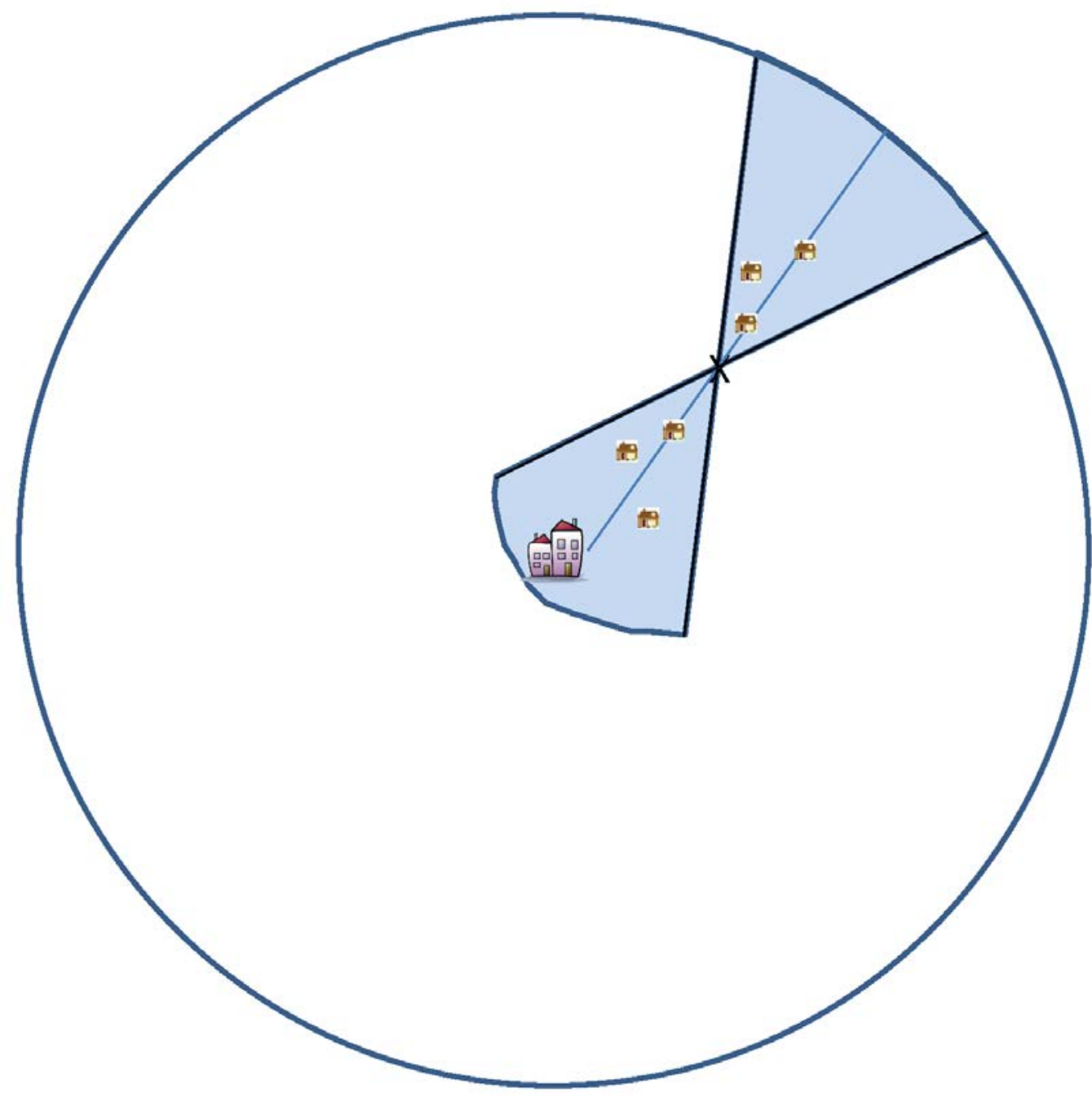

Note: LIHTC site is located in the middle of the ring. The site marked $\mathrm{X}$ is where the empirical derivative with respect to LIHTC distance is being measured. Houses inside the shaded region are candidates for the empirical derivative calculation. 
Figure 3: Average Price Impact of LIHTC

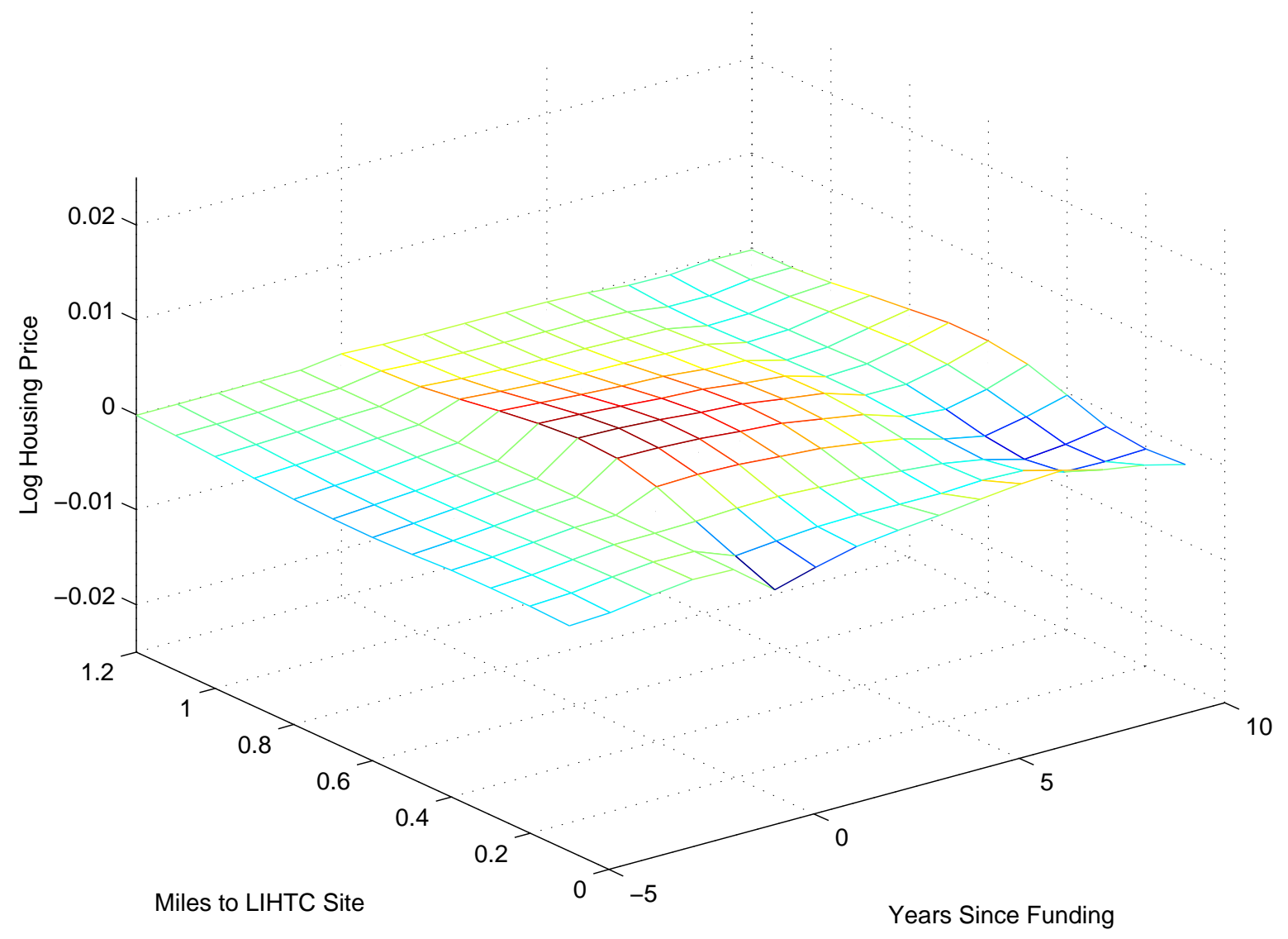

Note: Kernel smoothed estimates of log house prices using Nadaraya-Watson estimator with Epanechnikov kernel. Estimates integrate over the estimated derivatives to measure log price levels at a given distance from LIHTC site, relative to 1.4 miles away. 
Figure 4: Price Impact of LIHTC by Neighborhood Median Income

(a) Q1 Income Neighorhoods

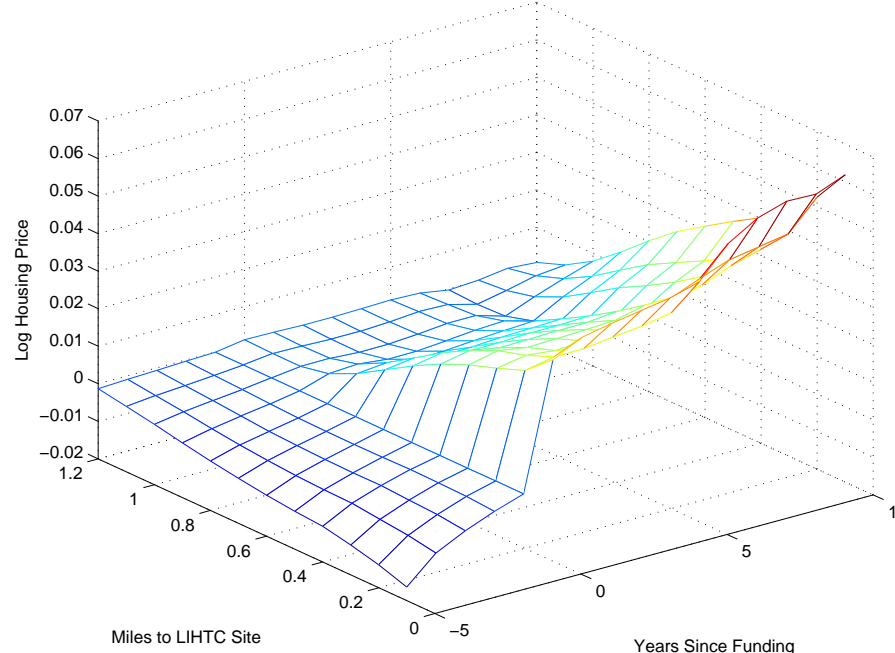

(c) Q3 Income Neighorhoods

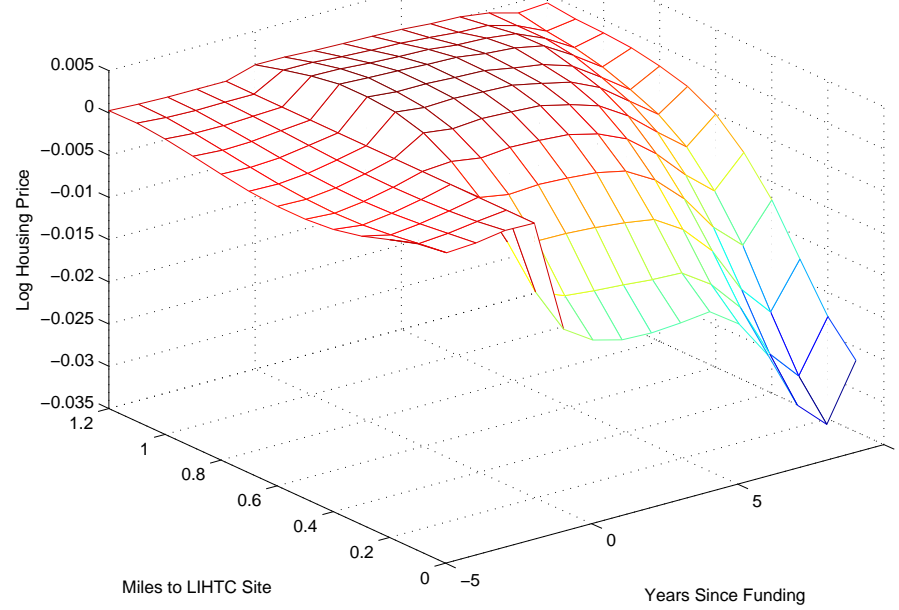

(b) Q2 Income Neighorhoods

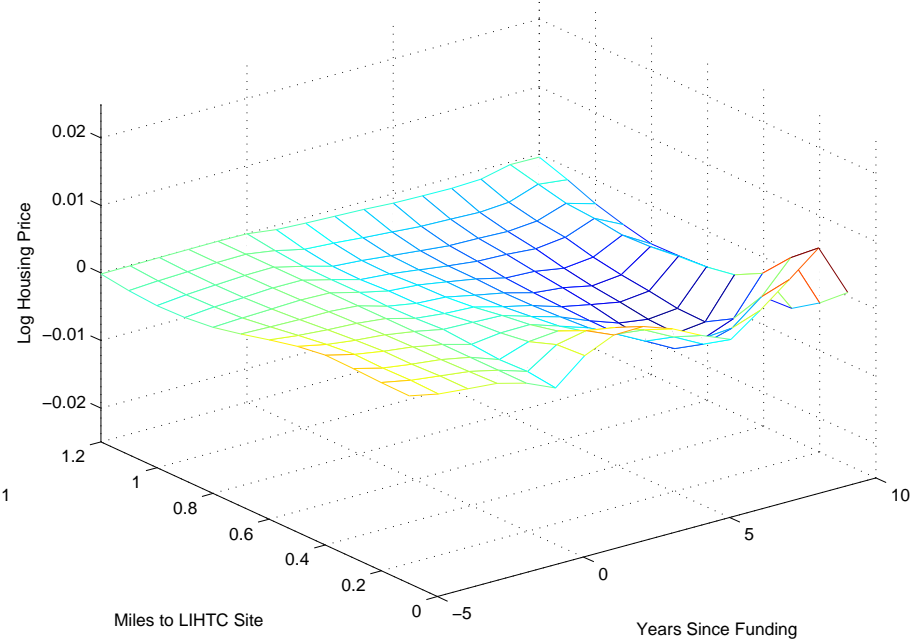

(d) Q4 Income Neighorhoods

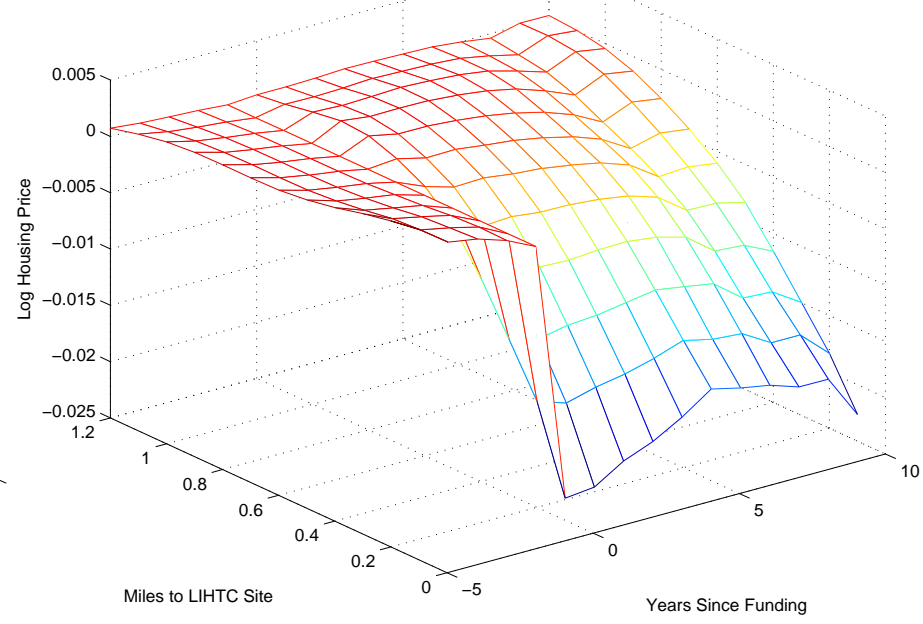

Note: Kernel smoothed estimates of log house prices using Nadaraya-Watson estimator with Epanechnikov kernel. Estimates integrate over the estimated derivatives to measure log price levels at a given distance from LIHTC site, relative to 1.4 miles away. Household median income quartile cutoffs are $\$ 26017, \$ 38177$, and $\$ 54642$ in 2012 dollars, as reported in the 1990 Census block group of the LIHTC site. 
Figure 5: Price Impact of LIHTC by Median Income within High Minority Neighborhoods

(a) Q1 \& Q2 Income, High Minority Neighorhoods

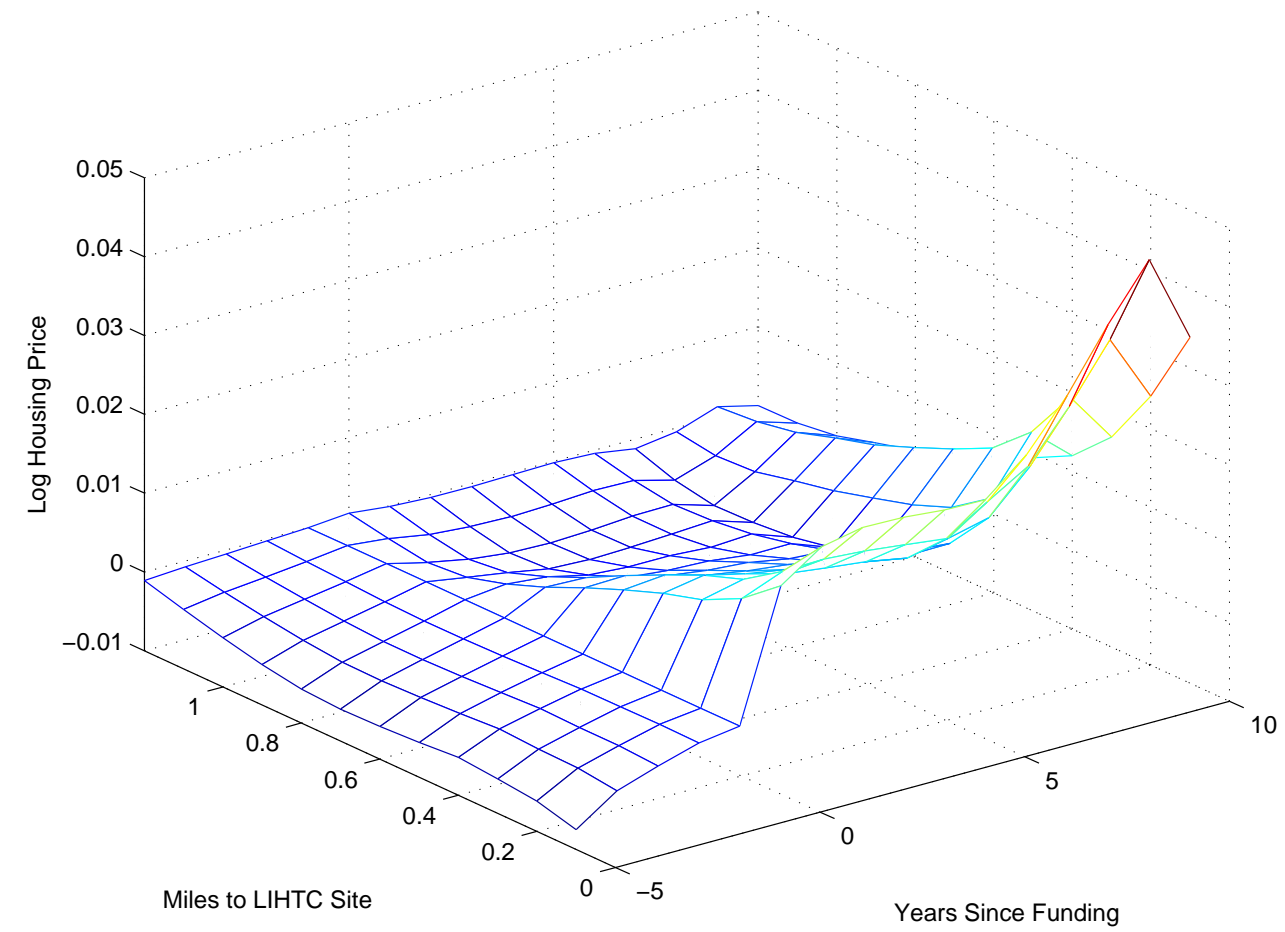

(b) Q3 \& Q4 Income, High Minority Neighorhoods

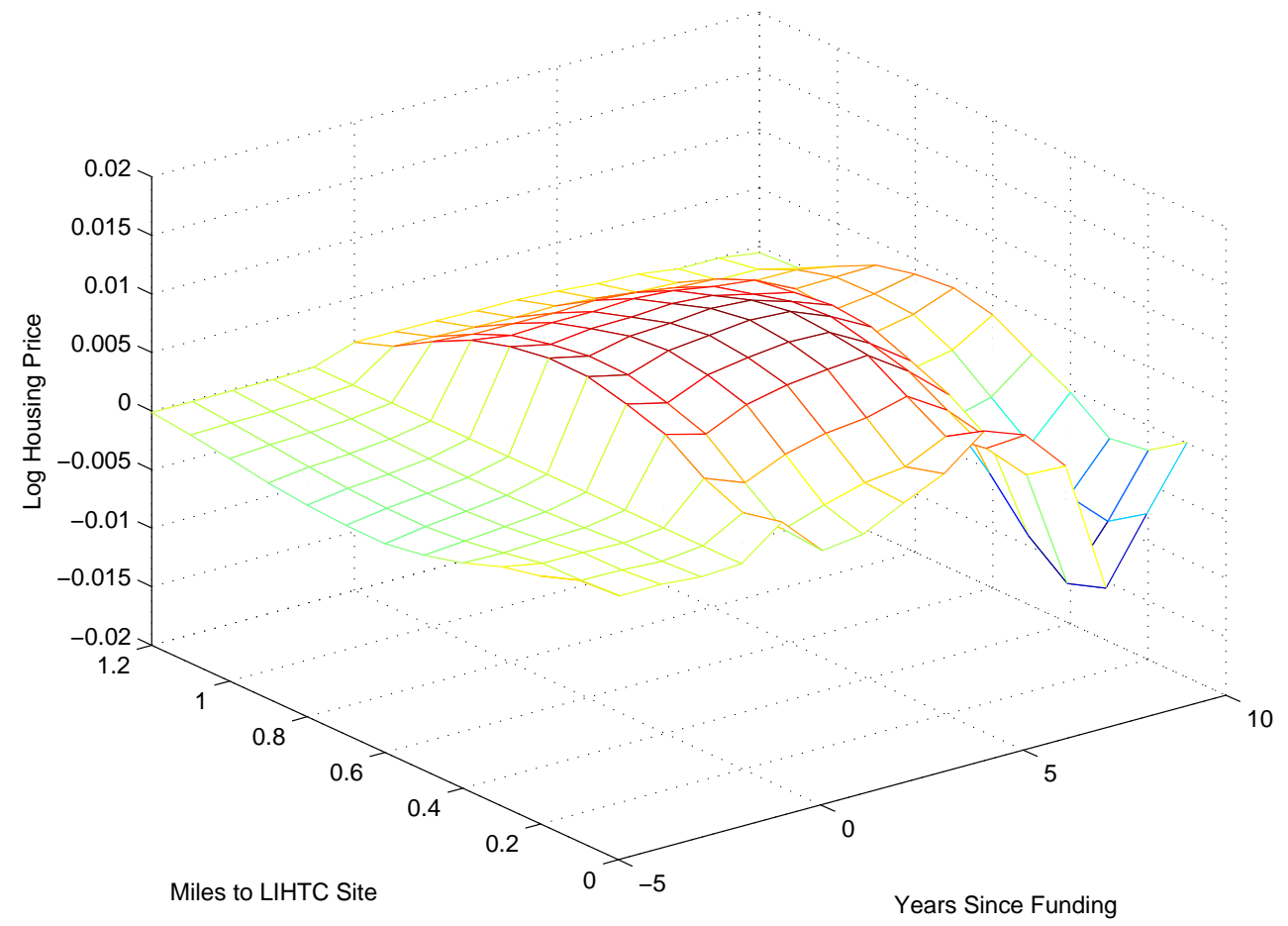

Note: Kernel smoothed estimates of log house prices using Nadaraya-Watson estimator with Epanechnikov kernel. Household median income quartile cutoffs are \$26017, \$38177, and \$54642 in 2012 dollars, as reported in the 1990 Census block group of the LIHTC site. 
Figure 6: Impact of LIHTC on Derivative of Log House Prices wrt miles from LIHTC vs miles to LIHTC:

Impacts 0 to 5 years post LIHTC Funding

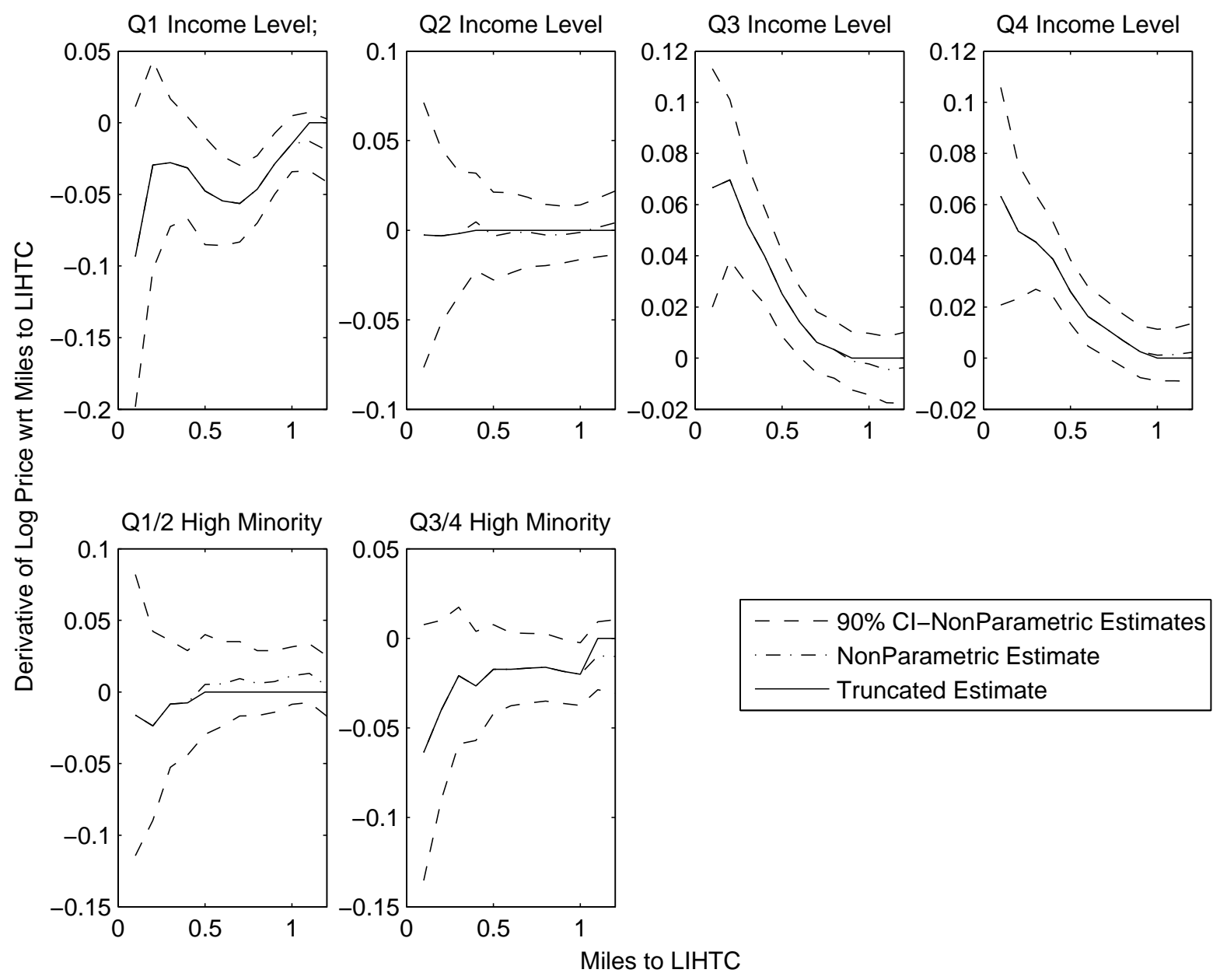

Note: Kernel smoothed estimates of log house price derivatives with respect to distance to LIHTC using Nadaraya-Watson estimator with Epanechnikov kernel. Household median income quartile cutoffs are $\$ 26017, \$ 38177$, and $\$ 54642$ in 2012 dollars, as reported in the 1990 Census block group of the LIHTC site. An LIHTC project is consider high minority share if at least $50 \%$ of the census block group where the LIHTC project is located was Black or Hispanic as reported in the 1990 census. Dashed lines are fully non-parametric estimate and confidence intervals. Solid line represents effect truncated to zero for distances beyond which the first time the non-parametric estimate crosses zero or gets closest to zero. These price effects are used in structural model of preference estimation. Standard errors estimated using a block-bootstrap with 500 simiulations where sampling is done over LIHTC sites. Dashed lines measure $90 \%$ confidence interval. 
Figure 7: Impact of LIHTC on Derivative of Log House Prices wrt miles from LIHTC vs miles to LIHTC:

Impacts 6 to 10 years post LIHTC Funding

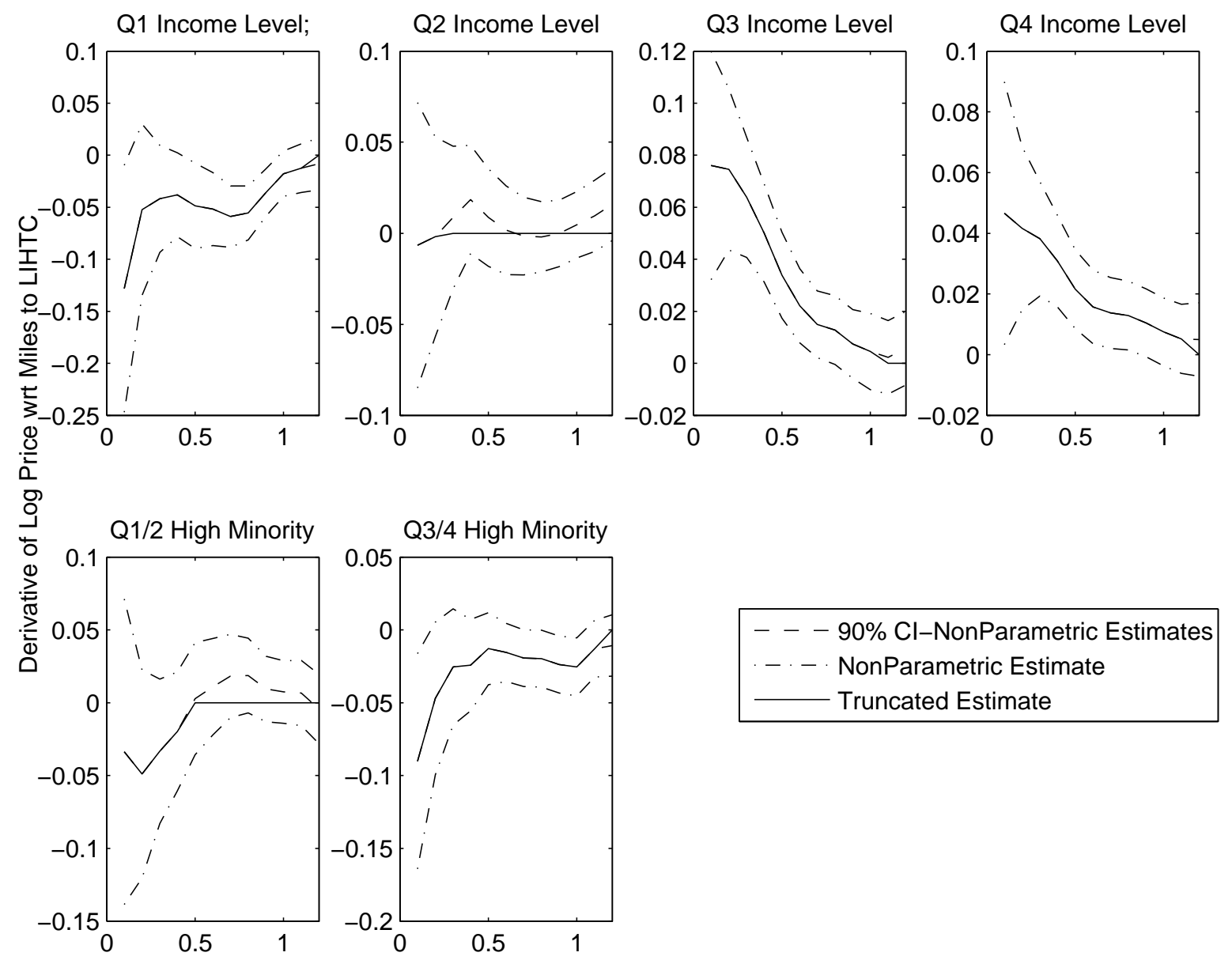

Note: Kernel smoothed estimates of log house price derivatives with respect to distance to LIHTC using Nadaraya-Watson estimator with Epanechnikov kernel. Household median income quartile cutoffs are $\$ 26017, \$ 38177$, and $\$ 54642$ in 2012 dollars, as reported in the 1990 Census block group of the LIHTC site. An LIHTC project is consider high minority share if at least $50 \%$ of the census block group where the LIHTC project is located was Black or Hispanic as reported in the 1990 census. Dashed lines are fully non-parametric estimate and confidence intervals. Solid line represents effect truncated to zero for distances beyond which the first time the non-parametric estimate crosses zero or gets closest to zero. These price effects are used in structural model of preference estimation. Standard errors estimated using a block-bootstrap with 500 simiulations where sampling is done over LIHTC sites. Dashed lines measure $90 \%$ confidence interval. 
Figure 8: Impact of LIHTC on Homebuyer Income:

Impacts 0 to 10 years post LIHTC Funding

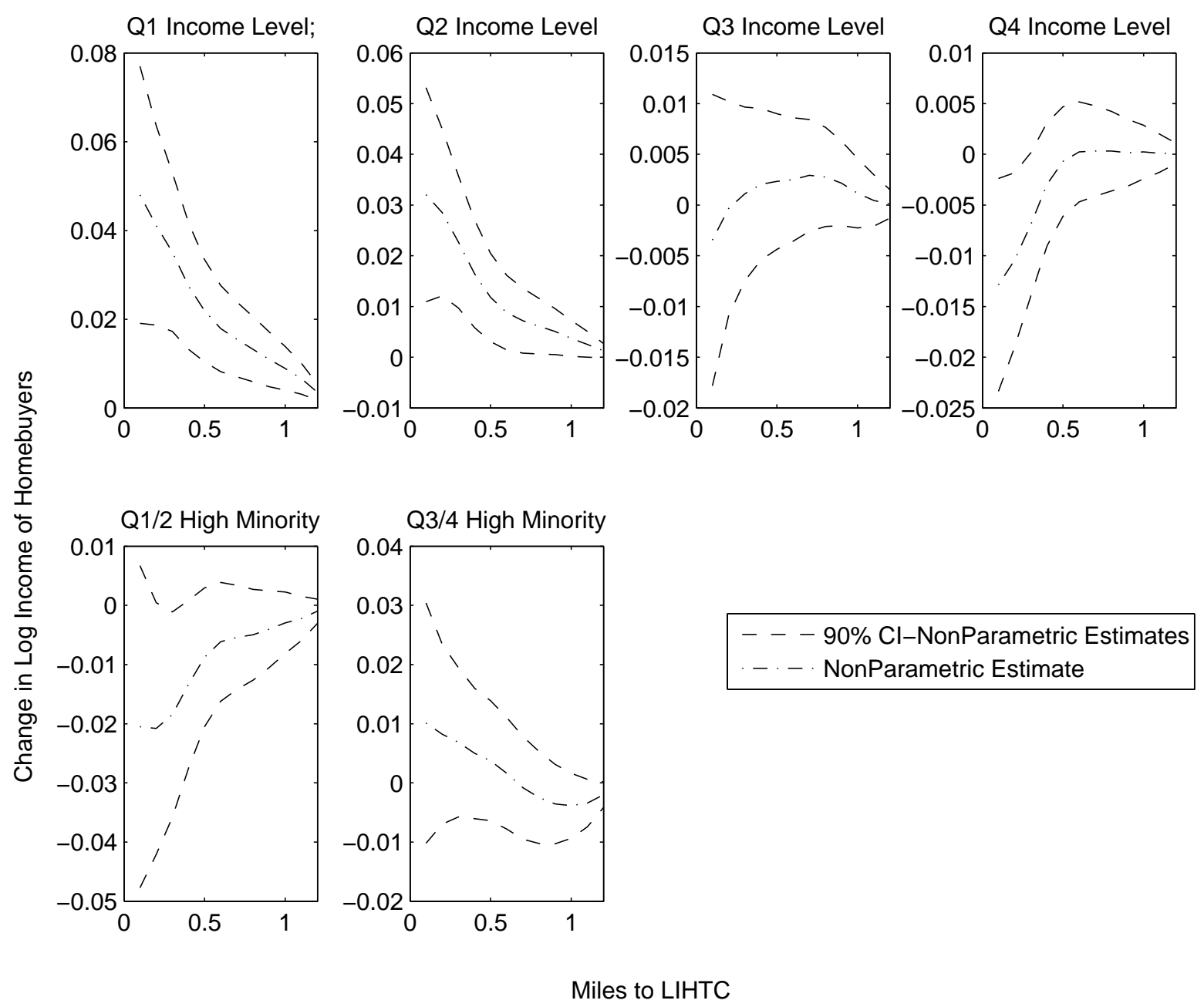

Note: Kernel smoothed estimates of log homebuyer income which are black using Nadaraya-Watson estimator with Epanechnikov kernel. Household median income quartile cutoffs are $\$ 26017, \$ 38177$, and $\$ 54642$ in 2012 dollars, as reported in the 1990 Census block group of the LIHTC site. An LIHTC project is consider high minority share if at least $50 \%$ of the census block group where the LIHTC project is located was Black or Hispanic as reported in the 1990 census. Dashed lines are fully non-parametric estimate and confidence intervals. Standard errors estimated using a blockbootstrap with 500 simiulations where sampling is done over LIHTC sites. Dashed lines measure $90 \%$ confidence interval. 
Figure 9: Impact of LIHTC on Black Share of Homebuyers:

Impacts 0 to 10 years post LIHTC Funding

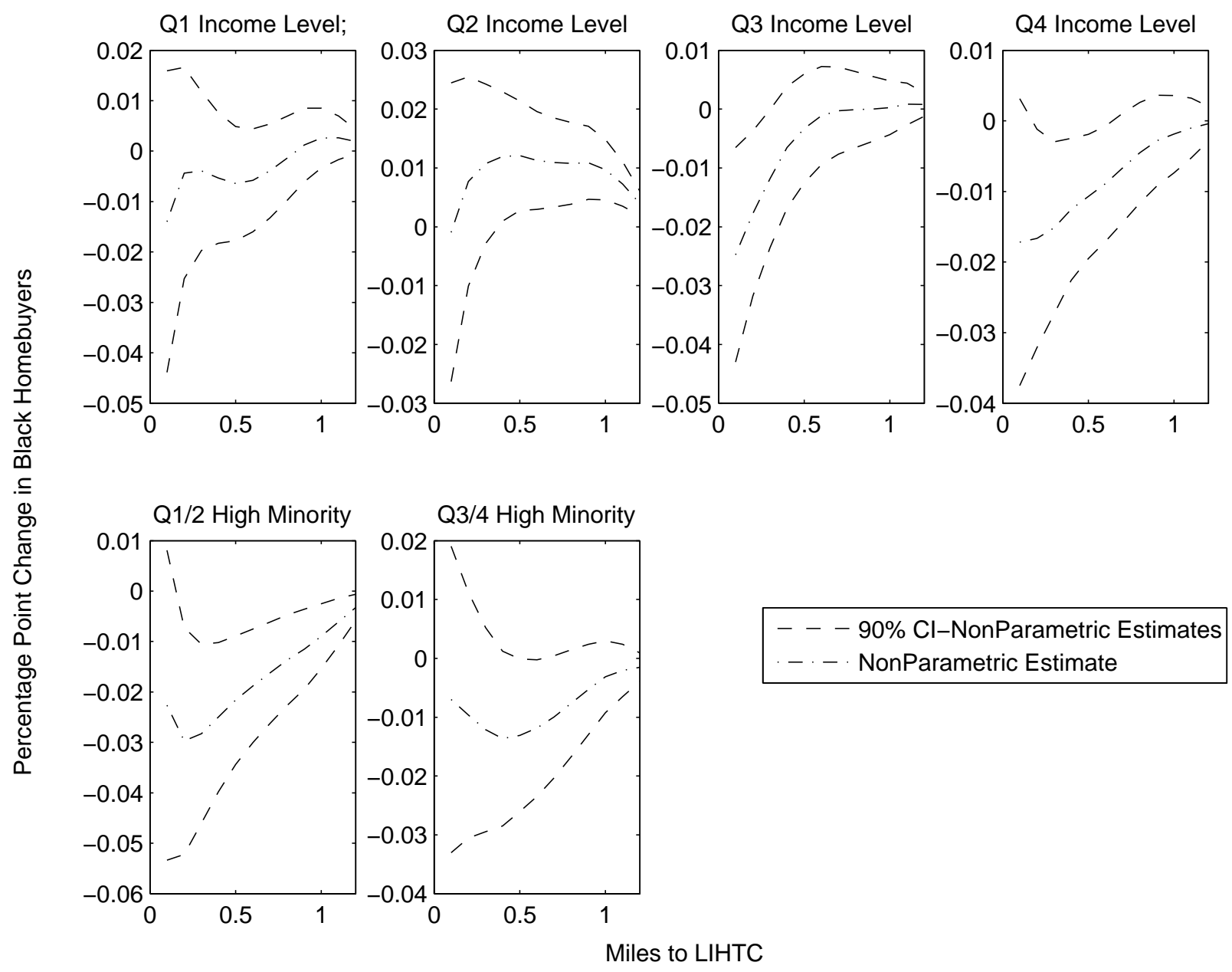

Note: Kernel smoothed estimates of percent of homebuyers which are black using Nadaraya-Watson estimator with Epanechnikov kernel. Household median income quartile cutoffs are $\$ 26017, \$ 38177$, and $\$ 54642$ in 2012 dollars, as reported in the 1990 Census block group of the LIHTC site. An LIHTC project is consider high minority share if at least $50 \%$ of the census block group where the LIHTC project is located was Black or Hispanic as reported in the 1990 census. Dashed lines are fully non-parametric estimate and confidence intervals. Solid line represents effect truncated to zero for distances beyond which the first time the non-parametric estimate crosses zero or gets closest to zero. These price effects are used in structural model of preference estimation. Standard errors estimated using a block-bootstrap with 500 simiulations where sampling is done over LIHTC sites. Dashed lines measure $90 \%$ confidence interval. 
Figure 10: Crime Impacts of LIHTC by Neighborhood Median Income

(a) Property Crime in High Minority Neighorhoods

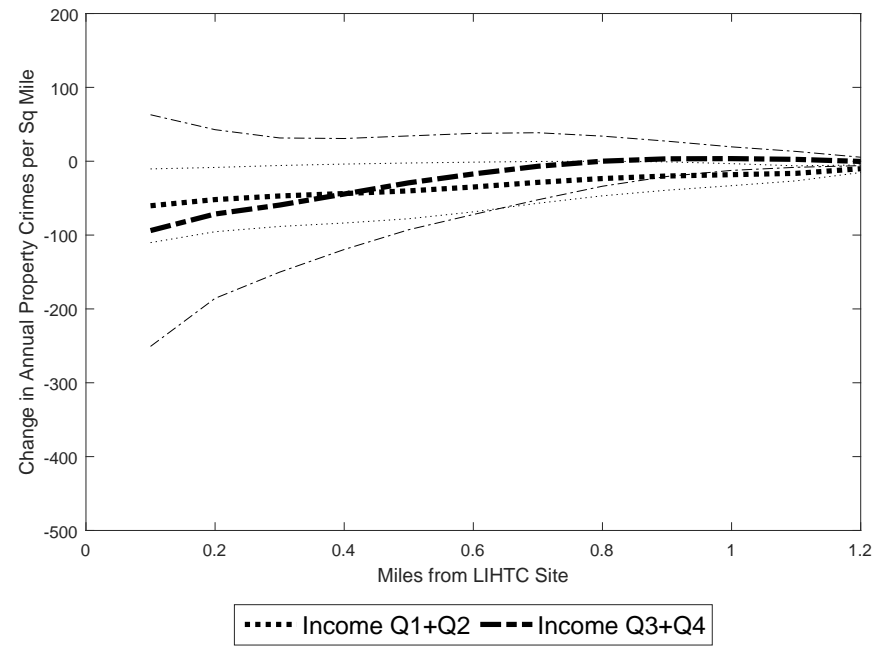

(c) Violent Crime in High Minority Neighorhoods

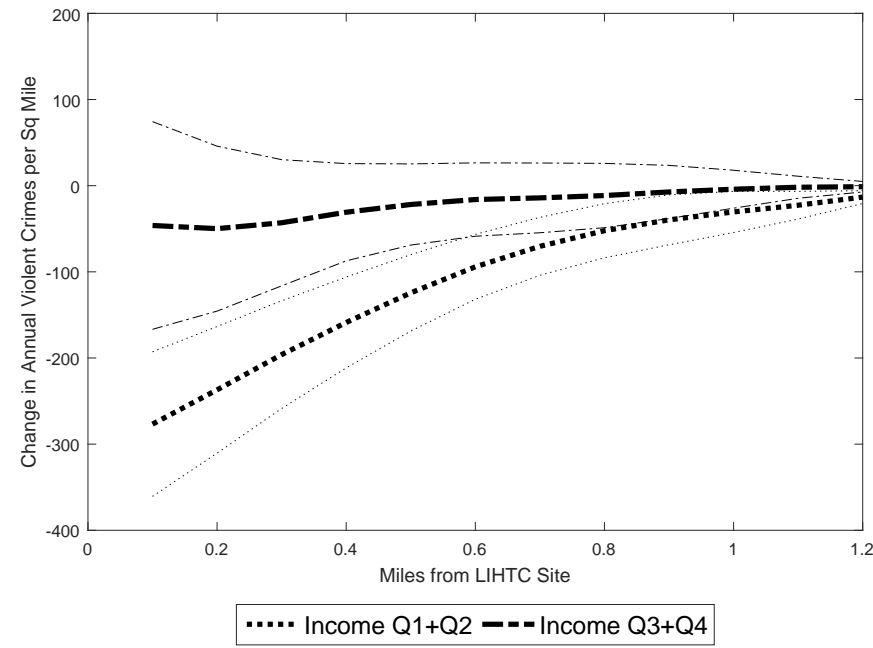

(b) Property Crime in Low Minority Neighorhoods

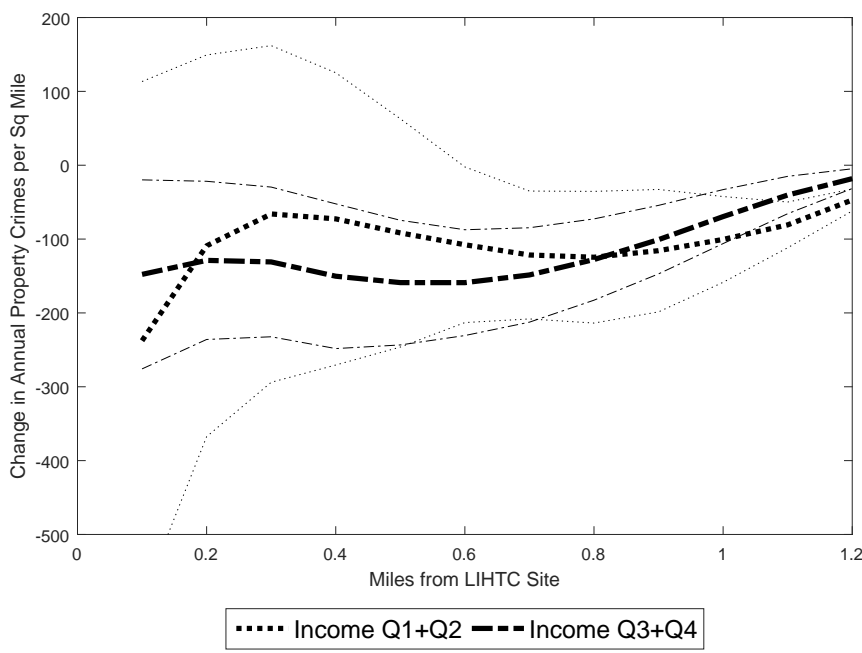

(d) Violent Crime in Low Minority Neighorhoods

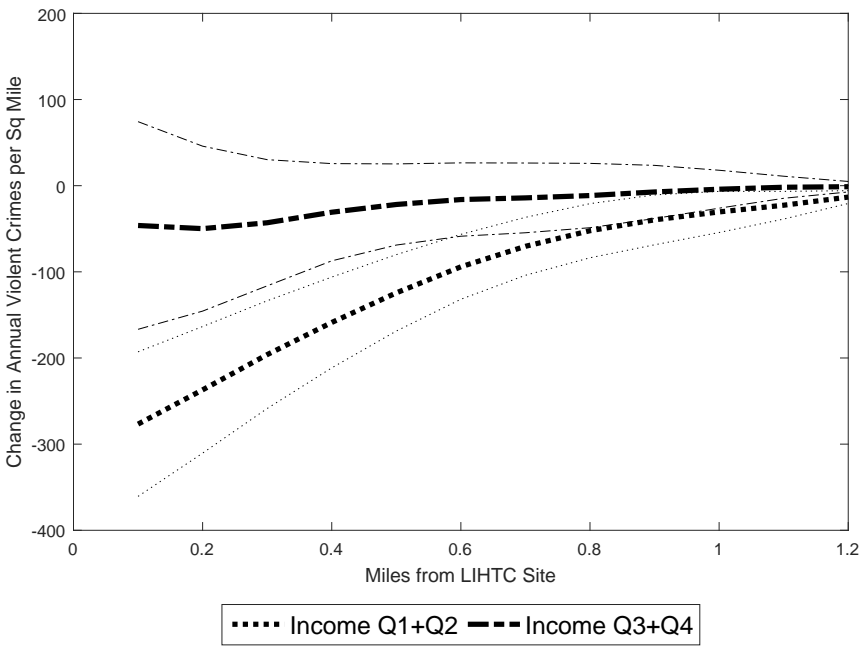

Note: Kernel smoothed estimates of annual crimes per square mile using Nadaraya-Watson estimator with Epanechnikov kernel. Estimates integrate over the estimated derivatives to measure log crimes per square mile at a given distance from LIHTC site, relative to 1.4 miles away. Household median income quartile cutoffs are $\$ 26017, \$ 38177$, and $\$ 54642$ in 2012 dollars, as reported in the 1990 Census block group of the LIHTC site. 
Figure 11: Mean Willingness to Pay to Live 0.1 miles from LIHTC: Low Minority Areas

(a) Q1 Income Neighorhoods

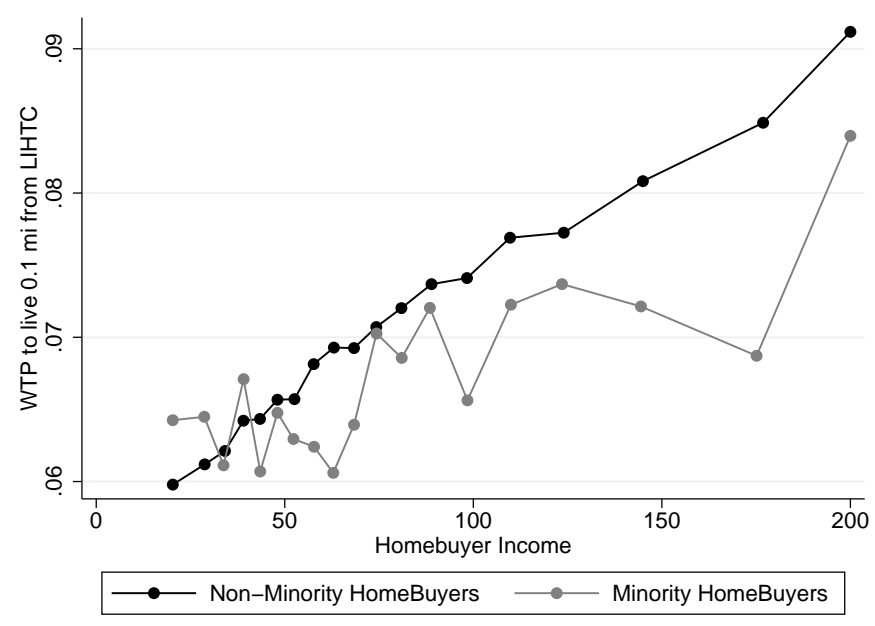

(c) Q3 Income Neighborhoods

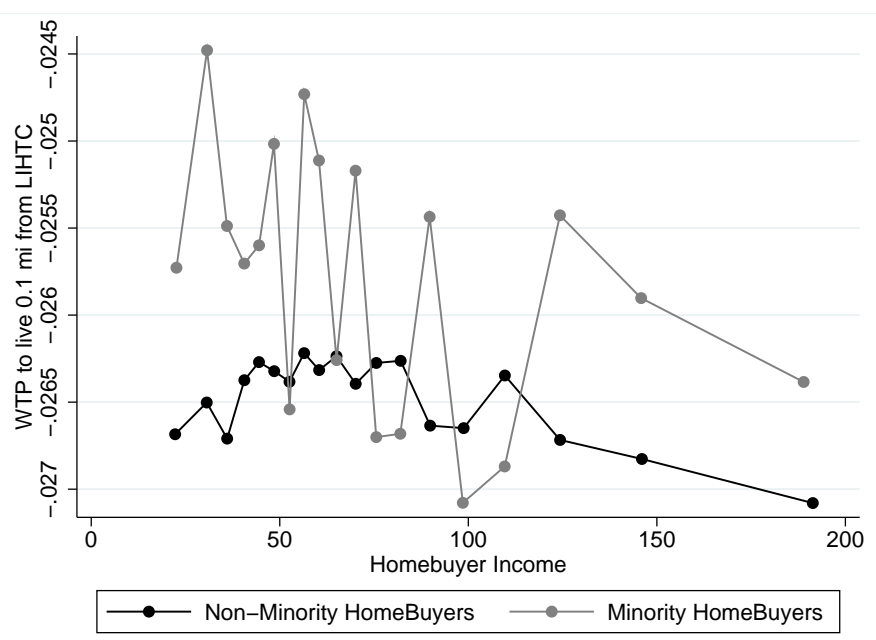

(b) Q2 Income Neighborhoods

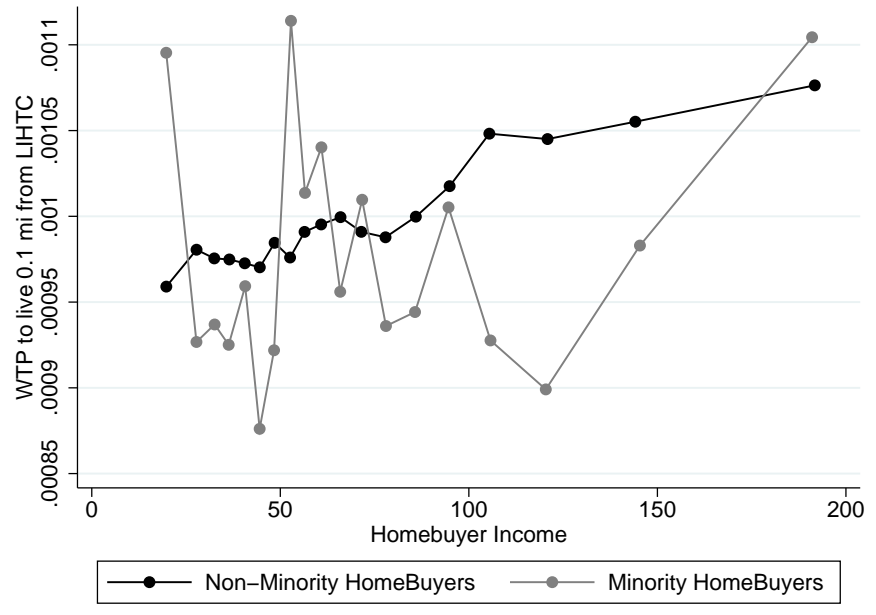

(d) Q4 Income Neighborhoods

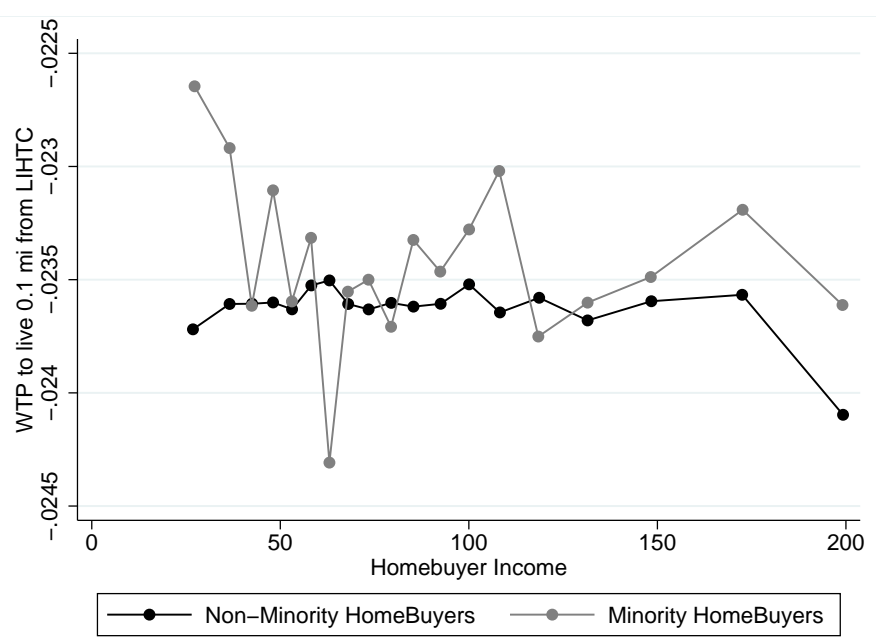

Note: Willingness to pay is measured as a percentage of the hombuyer's houseprice. Household median income quartile cutoffs are $\$ 26017, \$ 38177$, and $\$ 54642$ in 2012 dollars, as reported in the 1990 Census block group of the LIHTC site. Reported preferences are for households who choose to live close to LIHTC development. 
Figure 12: Mean Willingness to Pay to Live 0.1 miles from LIHTC: High Minority Areas

(a) Q1 Income Neighorhoods

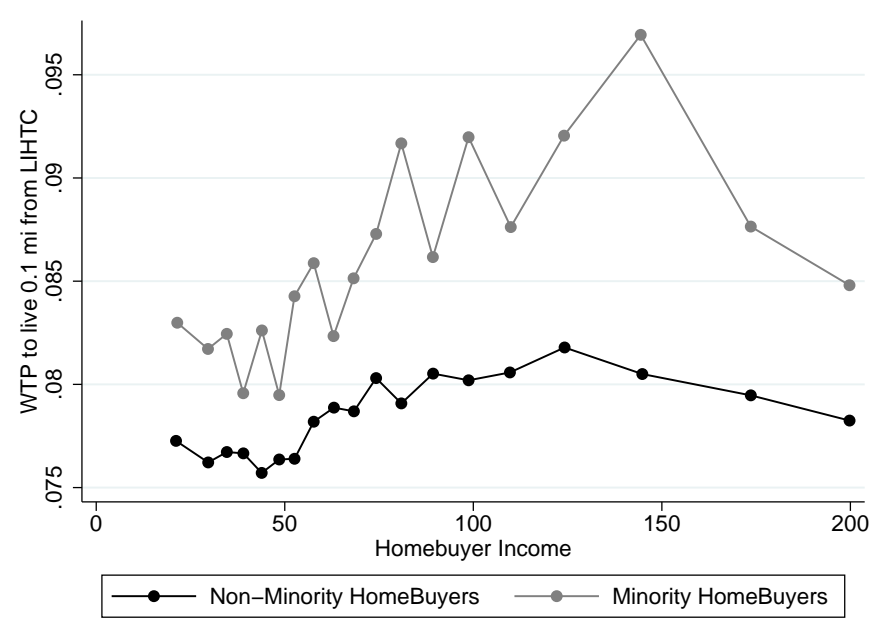

(c) Q3 Income Neighborhoods

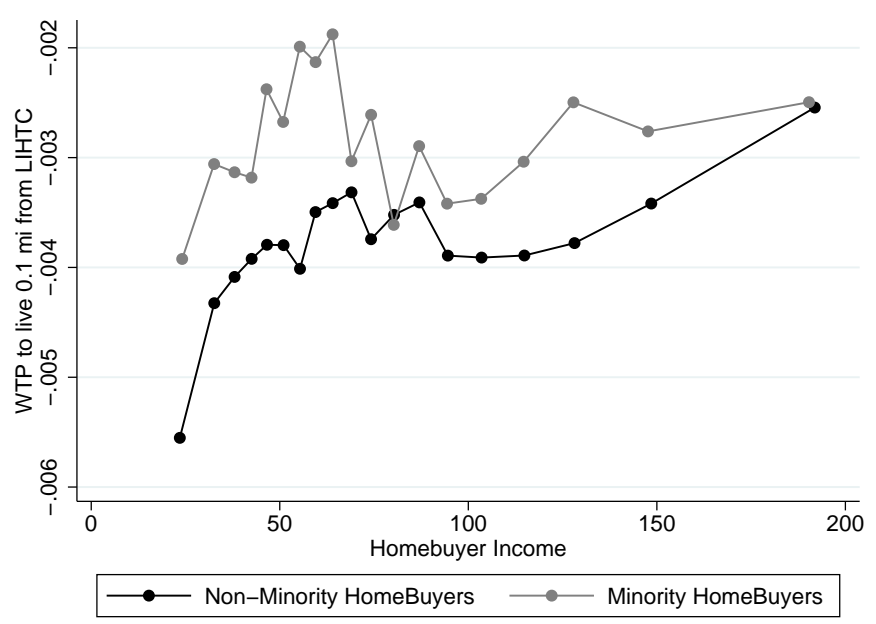

(b) Q2 Income Neighborhoods

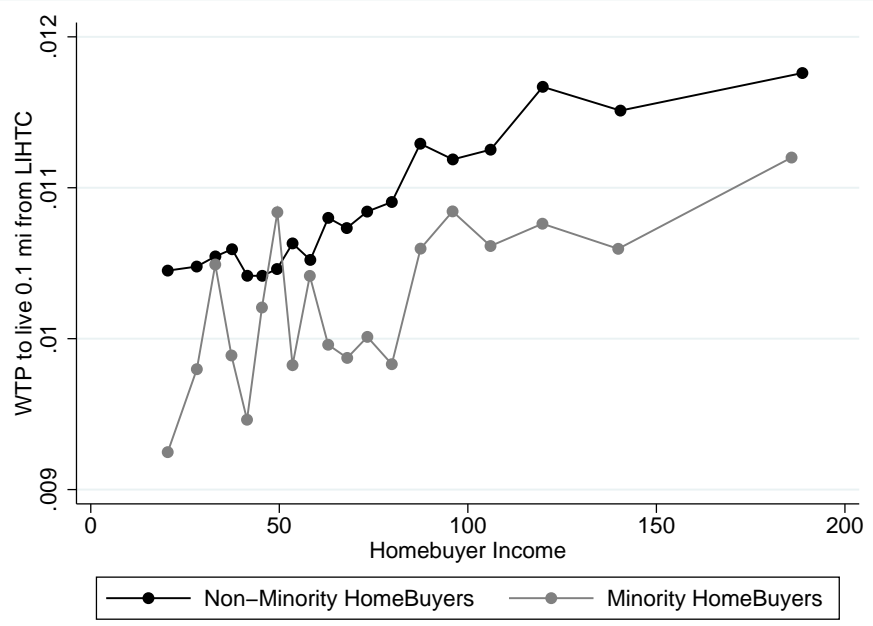

(d) Q4 Income Neighborhoods

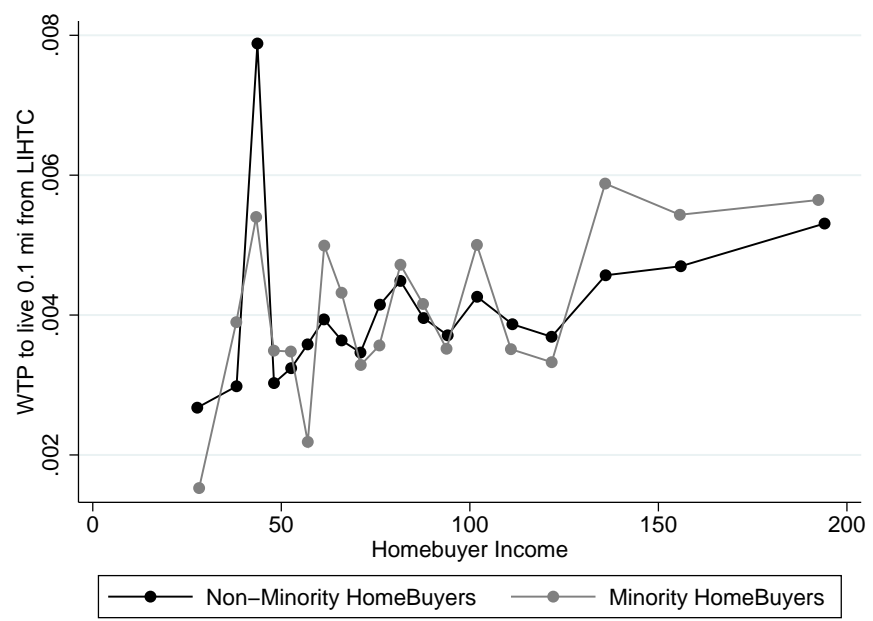

Note: Willingness to pay is measured as a percentage of the hombuyer's houseprice. Household median income quartile cutoffs are $\$ 26017, \$ 38177$, and $\$ 54642$ in 2012 dollars, as reported in the 1990 Census block group of the LIHTC site. Reported preferences are for households who choose to live close to LIHTC development. 
Table A1: Median \# of Households Impacted by LIHTC Development

Panel A: Low Minority Areas

\begin{tabular}{lcccc}
\hline & Income Q1 & Income Q2 & Income Q3 & Income Q4 \\
\hline \# Renting HHs Impacted by LIHTC & 4832.5 & 621 & 1171.5 & 1328 \\
\# Owning HHs Impacted by LIHTC & 2476 & 296 & 1042 & 2268 \\
\hline Observations & 658 & 884 & 1768 & 2463 \\
\hline & Panel B: High Minority Areas & & \\
\hline & Income Q1 & Income Q2 & Income Q3 & Income Q4 \\
\hline \# Renting HHs Impacted by LIHTC & 12171 & 2075 & 6028 & 5422 \\
\# Owning HHs Impacted by LIHTC & 3764.5 & 599 & 3630 & 4456.5 \\
\hline Observations & 2248 & 1817 & 1267 & 340 \\
\hline
\end{tabular}

Note: Median number of renting and home owning households living within the area impacted by LIHTC development, as meaured in the 2007-2012 ACS. Standard deviation in brackets below. Each observation is a neighborhood around a LIHTC development. 
Figure A1: Mean Willingness to Pay to Live 0.1 miles from LIHTC: Low Minority Areas

(a) Q1 Income Neighorhoods

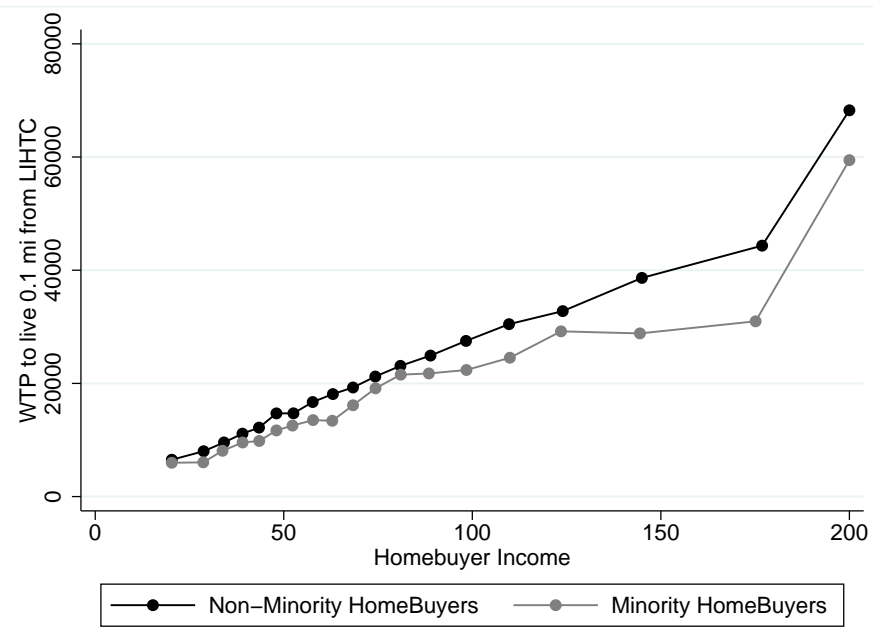

(c) Q3 Income Neighborhoods

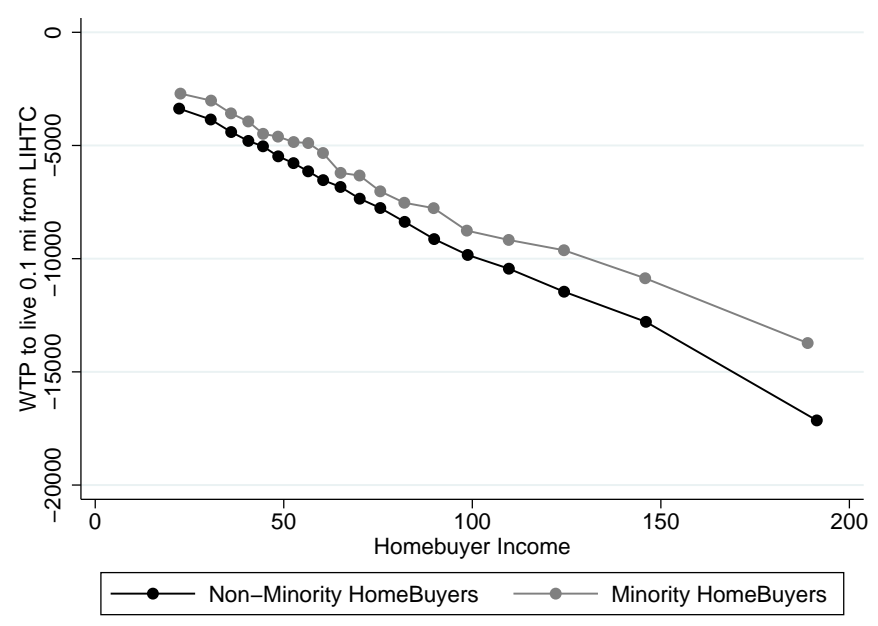

(b) Q2 Income Neighborhoods

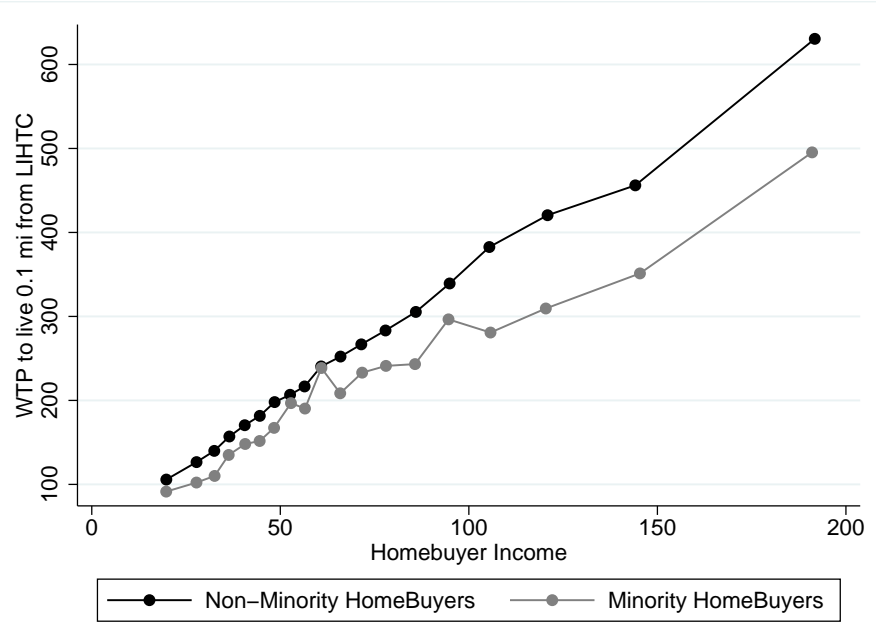

(d) Q4 Income Neighborhoods

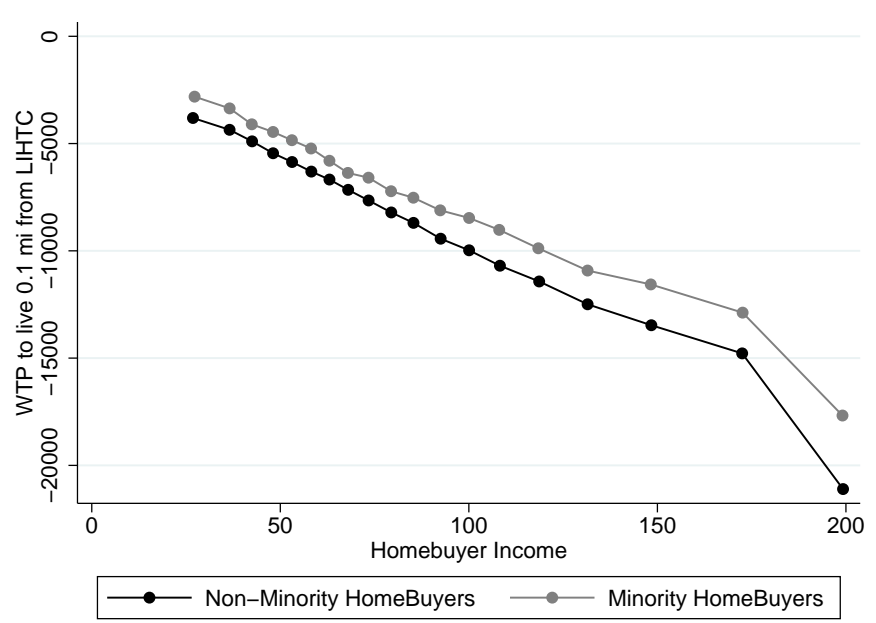

Note: Willingness to pay is measured in 2012 dollars. Household median income quartile cutoffs are $\$ 26017, \$ 38177$, and $\$ 54642$ in 2012 dollars, as reported in the 1990 Census block group of the LIHTC site. Reported preferences are for households who choose to live close to LIHTC development. 
Figure A2: Mean Willingness to Pay to Live 0.1 miles from LIHTC: High Minority Areas

(a) Q1 Income Neighorhoods

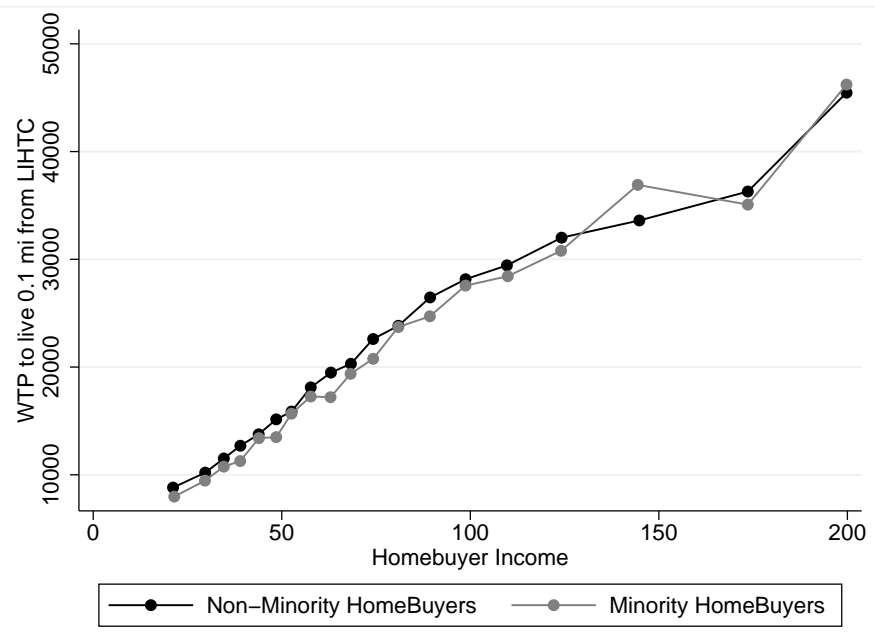

(c) Q3 Income Neighborhoods

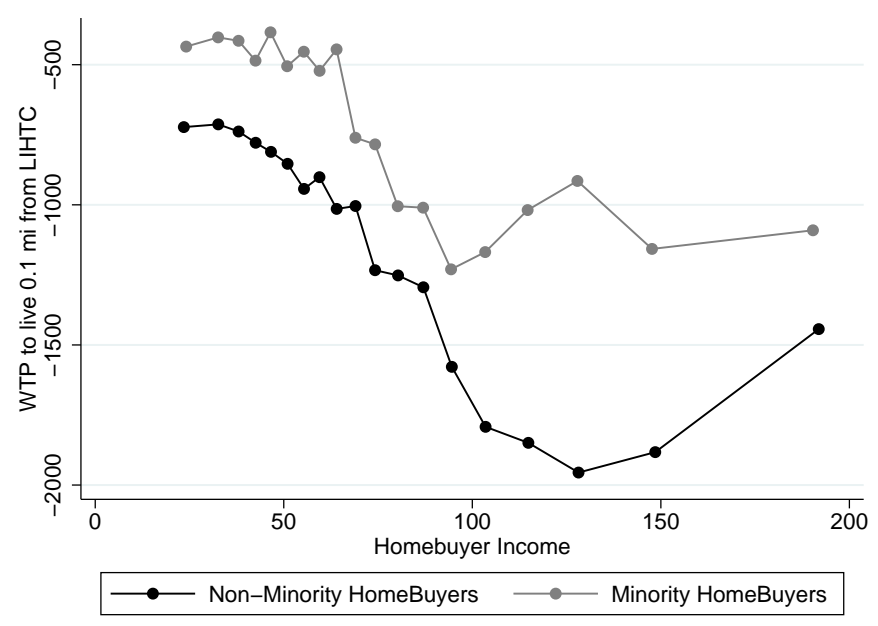

(b) Q2 Income Neighborhoods

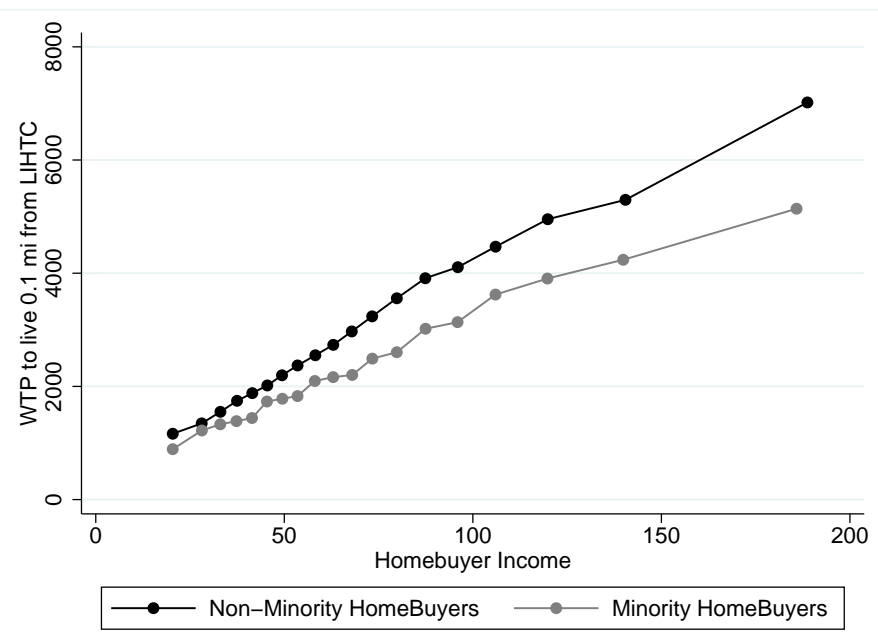

(d) Q4 Income Neighborhoods

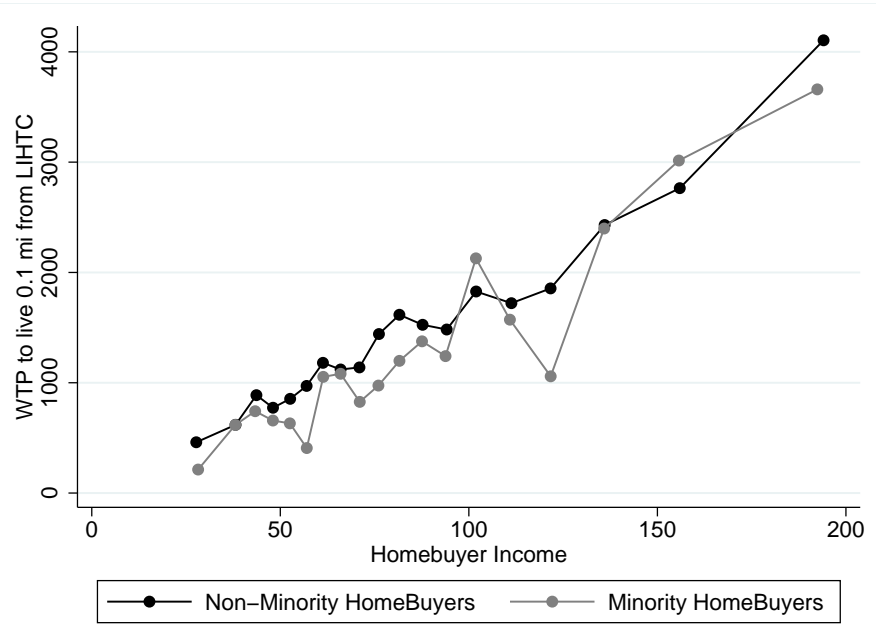

Note: Willingness to pay is measured in 2012 dollars. Household median income quartile cutoffs are $\$ 26017, \$ 38177$, and $\$ 54642$ in 2012 dollars, as reported in the 1990 Census block group of the LIHTC site. Reported preferences are for households who choose to live close to LIHTC development. 


\section{A Data \& Policy Appendix}

\section{A.1 The Low Income Housing Tax Credit}

In 1986, Congress passed the Tax Reform Act. As part of this legislation, Congress increased the Home Mortgage Interest Deduction and modified the treatment of imputed rent and local property taxes to further incentivize investment in owner-occupied housing. Concerned that such provisions would decrease the supply of affordable rental housing for low-income individuals, Congress introduced the Low Income Housing Tax Credit (LIHTC) as part of the Act to encourage the development of multifamily housing and thus serve as a balancing measure.

Each year, federal tax credits are allocated to the states based on population. In particular, each state receives the inflation-adjusted equivalent of $\$ 2.30$ per resident, as measured in 2014 dollars. These credits are awarded by state authorities to developers of qualified projects. Developers then sell these credits to investors to raise equity capital for their projects and reduce the amount of debt they would otherwise have to borrow. Investors receive a dollar-for-dollar credit against their federal tax liability for a period of 10 years, provided the property continues to comply with all program guidelines. ${ }^{38}$

To qualify for a tax credit under the Low Income Tax Credit Program, federal guidelines require that proposed projects be for construction or rehabilitation of a residential rental property and satisfy either one of two low-income occupancy criteria. At least 20 percent of tenants must earn less than 50 percent of the Area Median Gross Income (AGMI), or alternatively, at least 40 percent of tenants must earn less than 60 percent of AGMI. ${ }^{39}$ The AGMI is based on data from the Internal Revenue Service, the American Housing Survey, and the decennial Census. It is calculated annually by the Department of Housing and Urban Development (HUD) for all metropolitan areas and counties. If the income of a household in one of the low income units grows to exceed the relevant income limit, then the program requires developers to place a low income tenant into the next unit vacated by a market rate tenant. ${ }^{40}$ Additionally, developers must restrict rents, including utility allowance, in low-income units to 30 percent of the relevant income limit, i.e. either 50 percent of 60 percent of AGMI for a minimum affordability period of 30 years.

Note that these criteria are only the minimal requirements as specified by the federal government. In practice, states almost always receive many more project proposals and tax credit allocation requests from developers than they have federal allotments, generally on the order of 2 to 4 times. Each state is therefore required to maintain a "Qualified Application Plan" (QAP) to govern the selection process. These plans usually operate by assigning point scores to various project characteristics and then allocating tax credits based on point totals until funds are exhausted. Such project characteristics include tenant

\footnotetext{
${ }^{38}$ Eriksen (2009) studies the market pricing of these tax credits. He finds that that LIHTC developers in California received on average $\$ 0.73$ per $\$ 1$ of tax credit in the years 1999-2005.

${ }^{39}$ Actual income limits depend on household size. The 50 percent of AGMI limit is for a base family size of four members. Income limits are adjusted upward by 4 percentage points for each family member in excess of four. Limits are adjusted downward by 5 percentage points for each family member short of four. These limits are multiplied by 1.2 to get the 60 percent income limits.

${ }^{40}$ Many LITHC propties are comprised 100 percent of low-income units. Clearly, this requirement becomes superfluous in such a case.
} 
demographics, location, further funding sources, and structural properties of the building. Given this latitude the states enjoy in determining selection criteria, many require developers to go beyond the minimum number of affordable units and the minimum level of affordability.

The value of tax credits received by selected developers is calculated according to the project's "qualified basis", which essentially reflects the cost of constructing or rehabilitating the low-income units. Specifically, the project's "eligible basis" is the value of all depreciable development costs, such as construction, engineering, soil tests, and utility connection fees. It does not include land acquisition costs. The qualified basis is then calculated by multiplying the eligible basis by the "applicable fraction." This is the smaller of two percentages, the fraction of low income units in the development, or the fraction of total square footage allotted to low income units. Once the qualified basis has been determined, the annual tax credit is determined by applying the relevant housing tax credit rate. New construction or substantial rehabilitation projects, which are not otherwise subsidized by the Federal government, receive a 9 percent credit rate, while all other projects receive a 4 percent credit rate. These annual credits are then paid out over a period of 10 years. $^{41}$

Since its inception in 1986, the Low Income Housing Tax Credit Program has been an integral component in fostering the development of multifamily housing throughout the United States. With an annual tax credit valued at over 8 billion dollars, the program funded 21 percent of all multifamily developments between the years 1987-2008.

\section{A.2 Data}

\section{A.2.1 DataQuick}

Our first dataset is from DataQuick, which provides detailed public records housing characteristics and transactions data collected from county assessor and register of deeds officers. This dataset covers over 109 million properties from 1988-2012 in over 2,300 jurisdictions and provides information such as sales price, transaction type, loan amount, number of beds, number of baths, square-footage, lot size, age, etc. The quality of the DataQuick data is not uniform across the country. Certain states, such as Texas and Utah, do not require the prices of housing transactions to be a matter of public record. Thus, DataQuick does not report house prices for those states. Other states, such as Illinois, provide prices in their records but do not collect information regarding the number of bathrooms. Finally, not all of the counties covered by DataQuick have records dating back to 1988. Coverage of a significant number of counties began in 1996.

We restrict our analysis to those counties which have transactions history data dating to at least 1996. From this subset, we then restrict to those counties which have at least an average of 1000 residential arm-length sales per year. This leaves us with a sample of approximately 16 million transactions located within 1.5 miles of a LIHTC site in a total of 129 counties in 15 states, concentrated largely in the major metropolitan areas of New

\footnotetext{
${ }^{41}$ This calculation is a baseline figure. Congress passed legislation in 1989 affording state allocating agencies the option to increase the qualified basis by up to 30 percent in both "qualified census tracts" (QCTs) and "difficult development areas" (DDAs). Census tracts with 50 percent of households earning below 60 percent of AGMI earn qualified status, subject to a population restriction which is generally nonbinding. Metropolitan areas with high ratios of fair market rent to AMGI are designated as DDAs. See Baum-Snow and Marion (2009) for more details.
} 
England, Florida, California, Illinois, North Carolina, Tennessee, the Southwest and the Pacific Northwest. Figure 1 provides a map of the counties in our sample.

\section{A.2.2 Home Mortgage Disclosure Act Data}

We merge this dataset with data collected by the United States federal government according to the provisions of the Home Mortgage Disclosure Act (HMDA). Passed in 1975 due to concerns over redlining in the mortgage market in urban, minority areas, this legislation requires all lending institutions to report public loan data. Implemented as Regulation $\mathrm{C}$ by the Federal Reserve Board, it was amended in 1989 in response to the Financial Institutions Reform, Recovery, and Enforcement Act (FIRREA). ${ }^{42}$ These amendments greatly expanded the mortgage lenders covered under HMDA and required reporting of significant demographic information of both loan applicants and borrowers. The government provides public historical archives of this data covering the period 1991-2012. It includes information on loan census tract, loan amount, loan provider, and borrower demographics such as race, income, and sex. Since there is not a unique loan ID on which we can match the DataQuick data to the HMDA data, we perform a fuzzy merge. In particular, we merge the data according to census tract, year, loan amount, and bank name. This results in a match rate of approximately 80 percent. To examine whether our merge procedure linking the housing transaction data to the HMDA data introduces selection biases, Table 1 also reports house prices using both the DataQuick database alone as well as the merged DataQuick-HMDA database. The housing prices of those matched to the HMDA data are about 10\% higher than the average housing transaction, however this does not vary with distance to a LIHTC site.

\section{A.2.3 HUD LIHTC Database}

Information on LIHTC financed projects is provided by the Department of Housing and Urban Development (HUD). This data covers 39,094 projects and almost 2,458,00 low income housing units placed into service between the years 1987 and 2012. Note that the data therefore reflects only those projects approved by the state allocating agencies and not all project proposals submitted to them. The dataset includes detailed geographic information regarding the project location, the type of construction, the year the project was placed in service, the year funds for the project were allocated, and the number of units designated as low-income. Geocoded information is missing for some of the projects and we exclude from the sample any projects for which the year funds allocated variable is missing. We are left with 7098 LIHTC projects located in our sample of 129 counties. See Panel C of Table 1 for summary statistics. Due to DataQuick's coverage of counties, our sample is from more dense, urban areas, relative to the overall distribution of LIHTC sites.

\footnotetext{
${ }^{42}$ The rule-writing authority of Regulation $\mathrm{C}$ was transferred from the Federal Reserve Board to the Consumer Financial Protection Bureau on July 21, 2011.
} 


\section{B Econometric Proofs}

We begin by developing general econometric methods for estimating empirical derivatives. We will then apply these results to prove Theorem 1 in the main text. We develop our general econometric results by first focusing on the univariate case. This will ease exposition and provide intuition. Results for the multivariate case follow closely the logic of the univariate case.

\section{B.1 Univariate Case}

Suppose we draw an iid sample of size $n$ from the following nonparametric model

$$
Y_{i}=m\left(x_{i}\right)+\varepsilon_{i}
$$

where $E\left(\varepsilon_{i} \mid x_{i}\right)=0$ and $\operatorname{Var}\left(\varepsilon_{i} \mid x_{i}\right)=\sigma^{2}$. We assume that $m(x)$ is twice continuously differentiable. The variable $x_{i}$ is distributed according to the continuous density function:

$$
f(x):\left[x_{\min }, x_{\max }\right] \rightarrow(0, \infty) .
$$

We propose the following Nadaraya-Watson kernel estimator for the first derivative $m^{\prime}\left(x_{i}\right)$ :

$$
\begin{aligned}
\widehat{m}^{\prime}(x) & =\frac{n^{-1} \sum_{i=1}^{n} K_{h_{n}}\left(x-x_{i}\right) \tilde{Y}_{i}}{n^{-1} \sum_{i=1}^{n} K_{h_{n}}\left(x-x_{i}\right)} \\
\tilde{Y}_{i} & =\sum_{j=1}^{k_{n, i}} w_{j} \frac{Y_{i,+j}-Y_{i,-j}}{x_{i,+j}-x_{i,-j}} \mathbf{1}\left[k_{n, i}>0\right] \\
w_{j} & =\frac{j}{k_{n, i}\left(k_{n, i}+1\right) / 2}
\end{aligned}
$$

with the observations $\left\{\left(x_{i,+j}, Y_{i,+j}\right)\right\}_{j=1}^{k_{n, i}}\left\{\left(x_{i,-j}, Y_{i,-j}\right)\right\}_{j=1}^{k_{n, i}}$ defined recursively by:

$$
\begin{aligned}
& x_{i,+1}=\arg \min _{x>x_{i}+l_{n}} x, \quad x_{i,-1}=\arg \max _{x<x_{i}-l_{n}} x \\
& x_{i,+j}=\arg \min _{x>x_{i,+j-1}} x, \quad x_{i,-j}=\arg \max _{x<x_{i,-j-1}} x
\end{aligned}
$$

for $j=2, \ldots, k_{n, i}$ where $l_{n}>0$. Note that $Y_{i,+j}=m\left(x_{i,+j}\right)+\varepsilon_{i,+j}$. The random variable $k_{n, i}$ is defined as:

$$
\begin{aligned}
k_{n, i} & =\min \left(\left|L_{n, i}\right|,\left|U_{n, i}\right|, \kappa_{n}\right) \\
L_{i} & =\left\{x_{p}: x_{p}<x_{i}-l_{n}\right\} \\
U_{i} & =\left\{x_{p}: x_{p}>x_{i}-l_{n}\right\}
\end{aligned}
$$

for some $\kappa_{n}>0$. We define $K_{h_{n}}\left(x-x_{i}\right)$ as

$$
K_{h_{n}}\left(x-x_{i}\right)=\frac{1}{h_{n}} K\left(\frac{x-x_{i}}{h_{n}}\right),
$$


where $K(\cdot)$ is a kernel function. We have the following result:

Theorem 2 Assume the random design model above and suppose:

1. $K(u)$ is bounded, compactly supported, and symmetric

2. $n \rightarrow \infty, h_{n} \rightarrow 0, l_{n} \rightarrow 0, \kappa_{n} \rightarrow \infty$

3. $n h_{n} \rightarrow \infty, \kappa_{n} n^{-1} \rightarrow 0, l_{n}^{2} \kappa_{n} \rightarrow \infty$

Then $\widehat{m}^{\prime}(x) \rightarrow^{p} m^{\prime}(x)$ for all $x \in\left(x_{\min }, x_{\max }\right)$.

We prove the theorem through a sequence of lemmas. In what follows, we denote the indicator variable $\mathcal{I}_{n, i}=\mathbf{1}\left[k_{n, i}>0\right]$. We also denote $P_{i}(\cdot)=P\left(\cdot \mid x_{i}\right)$ and $E_{i}[\cdot]=E\left[\cdot \mid x_{i}\right]$.

Lemma 3 The estimate

$$
n^{-1} \sum_{i=1}^{n} K_{h_{n}}\left(x-x_{i}\right)
$$

converges in probability to $f(x)$ for all $x \in\left(x_{\min }, x_{\max }\right)$.

Proof. This is a standard result. See Hardle (1990).

Lemma 4 As $n \rightarrow \infty, P\left(k_{n, i}<\infty\right) \rightarrow 0$.

Proof. Trivial.

Lemma 5 Conditional on $x_{i}=u$ for any $u \in\left(x_{\min }, x_{\max }\right)$, as $n \rightarrow \infty$ the difference $\left(x_{i,+k_{n, i}}-x_{i,-k_{n, i}}\right) \mathcal{I}_{n, i}$ converges in probability to zero.

Proof. Fix $\delta>0$. Since $l_{n} \rightarrow 0$, there exists $N_{1}$ such that for all $n>N_{1}, l_{n}<\delta / 4$. Conditional on $x_{i}=u$, we have:

$$
P_{i}\left(\left(x_{i,+k_{n, i}}-x_{i,-k_{n, i}}\right) \mathcal{I}_{n, i}>\delta\right) \leq F_{B}\left(2 \kappa_{n} ; n-1, p_{\delta}\right)
$$

where $F_{B}\left(\cdot ; n-1, p_{\delta}\right)$ denotes the binomial distribution and

$$
p_{\delta}=\int_{u-\delta / 2}^{u-\delta / 4} f(s) d s+\int_{u+\delta / 4}^{u+\delta / 2} f(s) d s>0 .
$$

Since $\kappa_{n} n^{-1} \rightarrow 0$ as $n \rightarrow \infty$, there exists $N_{2}$ such that $\kappa_{n} n^{-1}<p_{\delta} / 4$ for all $n \geq N_{2}$. Let $N=\max \left\{N_{1}, N_{2},-2 \ln (\delta) / p_{\delta}^{2}\right\}$. Then by Hoeffding's inequality, for all $n>N$ :

$$
\begin{aligned}
P_{i}\left(\left(x_{i,+k_{n, i}}-x_{i,-k_{n, i}}\right) \mathcal{I}_{n, i}>\delta\right) & \leq \exp \left(-2 \frac{\left((n-1) p_{\delta}-2 \kappa_{n}\right)^{2}}{n-1}\right) \\
& \leq \exp \left(-\frac{(n-1) p_{\delta}^{2}}{2}\right) \\
& \leq \delta
\end{aligned}
$$

which proves the claim. 
Lemma 6 As $n \rightarrow \infty$, the bias:

$$
\left|E\left[\frac{1}{n} \sum_{i=1}^{n} K_{h_{n}}\left(x-x_{i}\right) \tilde{Y}_{i}\right]-m^{\prime}(x) f(x)\right| \rightarrow 0 .
$$

Proof. The idea of the proof is to apply a first-order Taylor expansion to the empirical derivatives. We then must show that the remainder term in the expansion converges to zero as the sample size grows. We can write the bias of the estimator as:

$$
\begin{aligned}
& \left|E\left[\frac{1}{n} \sum_{i=1}^{n} K_{h_{n}}\left(x-x_{i}\right) \tilde{Y}_{i}\right]-m^{\prime}(x) f(x)\right| \\
= & \left|E\left[\frac{1}{n} \sum_{i=1}^{n} K_{h_{n}}\left(x-x_{i}\right) \sum_{j=1}^{k_{n, i}} w_{j} \frac{Y_{i,+j}-Y_{i,-j}}{x_{i,+j}-x_{i,-j}} \mathcal{I}_{n, i}\right]-m^{\prime}(x) f(x)\right|
\end{aligned}
$$

Denote expectation conditional on the observed sample covariates as $E_{x}[\cdot]$. By applying Taylor's theorem, we have

$$
\begin{aligned}
& E_{x}\left[\sum_{j=1}^{k_{n, i}} \frac{Y_{i,+j}-Y_{i,-j}}{x_{i,+j}-x_{i,-j}} \mathcal{I}_{n, i}\right] \\
= & m^{\prime}\left(x_{i}\right) \sum_{j=1}^{k_{n, i}} w_{j} \frac{x_{i,+j}-x_{i,-j}}{x_{i,+j}-x_{i,-j}} \mathcal{I}_{n, i} \\
& +\frac{1}{2} \sum_{j=1}^{k_{n, i}} w_{j} \frac{m^{\prime \prime}\left(\zeta_{i, i+j}\right)\left(x_{i,+j}-x_{i}\right)^{2}-m^{\prime \prime}\left(\zeta_{i, i-j}\right)\left(x_{i}-x_{i,-j}\right)^{2}}{x_{i,+j}-x_{i,-j}} \mathcal{I}_{n, i} \\
= & m^{\prime}\left(x_{i}\right) \mathcal{I}_{n, i}+\frac{1}{2} \sum_{j=1}^{k_{n, i}} w_{j} \frac{m^{\prime \prime}\left(\zeta_{i, i+j}\right)\left(x_{i,+j}-x_{i}\right)^{2}-m^{\prime \prime}\left(\zeta_{i, i-j}\right)\left(x_{i}-x_{i,-j}\right)^{2}}{x_{i,+j}-x_{i,-j}} \mathcal{I}_{n, i}
\end{aligned}
$$

where $\zeta_{i, i+j} \in\left(x_{i}, x_{i,+j}\right)$ and $\zeta_{i, i-j} \in\left(x_{i,-j}, x_{i}\right)$. By the law of iterated expectations and the triangle inequality, the bias is thus bounded above by

$$
\begin{aligned}
& \left|E\left[\frac{1}{n} \sum_{i=1}^{n} K_{h_{n}}\left(x-x_{i}\right) m^{\prime}\left(x_{i}\right) \mathcal{I}_{n, i}\right]-m^{\prime}(x) f(x)\right| \\
& +\left|E\left[\frac{1}{n} \sum_{i=1}^{n} K_{h_{n}}\left(x-x_{i}\right) \sum_{j=1}^{k_{n, i}} w_{j} \frac{m^{\prime \prime}\left(\zeta_{i, i+j}\right)\left(x_{i,+j}-x_{i}\right)^{2}-m^{\prime \prime}\left(\zeta_{i, i-j}\right)\left(x_{i}-x_{i,-j}\right)^{2}}{x_{i,+j}-x_{i,-j}} \mathcal{I}_{n, i}\right]\right| .
\end{aligned}
$$

We know that that $P\left(k_{n, i}>0\right) \rightarrow 1$. The first term thus goes to zero as $n \rightarrow \infty$ according to the usual proof for consistency of Nadaraya-Watson estimators. See, for example, Hardle (1990). To show that the second term converges to zero, note first that it is bounded above 
by: ${ }^{43}$

$$
\begin{aligned}
& \sup _{x}\left|m^{\prime \prime}(x)\right| \int\left|K_{h_{n}}\left(x-x_{i}\right)\right|\left|E_{i}\left[\sum_{j=1}^{k_{n, i}} w_{j} \frac{\left(x_{i,+j}-x_{i}\right)^{2}+\left(x_{i}-x_{i,-j}\right)^{2}}{x_{i,+j}-x_{i,-j}} \mathcal{I}_{n, i}\right]\right| f\left(x_{i}\right) d x_{i} \\
\leq & \sup _{x}\left|m^{\prime \prime}(x)\right| \int\left|K_{h_{n}}\left(x-x_{i}\right)\right| E_{i}\left[\sum_{j=1}^{k_{n, i}} w_{j} \frac{\left(x_{i,+j}-x_{i}\right)^{2}+\left(x_{i}-x_{i,-j}\right)^{2}}{x_{i,+j}-x_{i,-j}} \mathcal{I}_{n, i}\right] f\left(x_{i}\right) d x_{i} \\
\leq & \sup _{x}\left|m^{\prime \prime}(x)\right| \int\left|K_{h_{n}}\left(x-x_{i}\right)\right| E_{i}\left[\sum_{j=1}^{k_{n, i}} w_{j}\left(x_{i,+j}-x_{i,-j}\right) \mathcal{I}_{n, i}\right] f\left(x_{i}\right) d x_{i} \\
\leq & \sup _{x}\left|m^{\prime \prime}(x)\right| \int\left|K_{h_{n}}\left(x-x_{i}\right)\right| E_{i}\left[\left(x_{i,+k_{n, i}}-x_{i,-k_{n, i}}\right) \mathcal{I}_{n, i}\right] f\left(x_{i}\right) d x_{i}
\end{aligned}
$$

By Hardle (1990), it thus suffices to show that $E_{i}\left[\left(x_{i,+k_{n, i}}-x_{i,-k_{n, i}}\right) \mathcal{I}_{n, i}\right]$ converges to zero. But we know by Lemma $5, P_{i}\left(\left(x_{i,+k_{n, i}}-x_{i,-k_{n, i}}\right) \mathcal{I}_{n, i}>0\right)$ converges to zero. Since $\left(x_{i,+k_{n, i}}-x_{i,-k_{n, i}}\right) \mathcal{I}_{n, i}$ is almost surely bounded, convergence in probability implies $E_{i}\left[\left(x_{i,+k_{n, i}}-x_{i,-k_{n, i}}\right) \mathcal{I}_{r}\right.$ converges to zero as well. This completes the proof.

Lemma 7 As $n \rightarrow \infty$, the variance:

$$
\operatorname{Var}\left(n^{-1} \sum_{i=1}^{n} K_{h_{n}}\left(x-x_{i}\right) \widetilde{Y}_{i}\right) \rightarrow 0
$$

Proof. The variance can be decomposed as:

$$
\frac{1}{n} \operatorname{Var}\left[K_{h_{n}}\left(x-x_{i}\right) \tilde{Y}_{i}\right]+\frac{1}{n^{2}} \sum_{i \neq j} \operatorname{Cov}\left(K_{h_{n}}\left(x-x_{i}\right) \widetilde{Y}_{i}, K_{h_{n}}\left(x-x_{j}\right) \widetilde{Y}_{j}\right)
$$

By the law of total variance:

$$
\begin{aligned}
\frac{1}{n} \operatorname{Var}\left[K_{h_{n}}\left(x-x_{i}\right) \tilde{Y}_{i}\right] & =\frac{1}{n}\left[E\left[\operatorname{Var}_{i}\left(K_{h_{n}}\left(x-x_{i}\right) \tilde{Y}_{i}\right)\right]+\operatorname{Var}\left(E_{i}\left[K_{h_{n}}\left(x-x_{i}\right) \tilde{Y}_{i}\right]\right)\right] \\
& \rightarrow \frac{1}{n} E\left[K_{h_{n}}^{2}\left(x-x_{i}\right) \operatorname{Var}_{i}\left(\widetilde{Y}_{i}\right)\right]+\frac{1}{n} \operatorname{Var}\left(K_{h_{n}}\left(x-x_{i}\right) m^{\prime}\left(x_{i}\right)\right)
\end{aligned}
$$

The second term approaches zero as $n \rightarrow \infty$ by Hardle (1990). Also by Hardle (1990), for the first term it suffices to show that:

$$
\operatorname{Var}_{i}\left(\widetilde{Y}_{i}\right) \rightarrow 0
$$

The idea of the proof is to again expand the empirical derivatives using Taylor's theorem. We then use the law of total variance to condition on the observables. We then prove that

\footnotetext{
${ }^{43}$ Recall that $E_{i}[\cdot]=E\left[\cdot \mid x_{i}=u\right]$.
} 
the variance converges to zero. Applying a Taylor expansion, the variance becomes:

$$
\operatorname{Var}_{i}\left(\begin{array}{c}
\sum_{j=1}^{k_{n, i}} w_{j} \frac{\varepsilon_{i,+j}-\varepsilon_{i, j}}{x_{i,+j}-x_{i,-j}} \mathcal{I}_{n, i} \\
+\sum_{j=1}^{k_{n, i}} w_{j} \frac{m^{\prime \prime}\left(\zeta_{i, i+j}\right)\left(x_{i,+j}-x_{i}\right)^{2}-m^{\prime \prime}\left(\zeta_{i, i-j}\right)\left(x_{i}-x_{i,-j}\right)^{2}}{x_{i,+j}-x_{i,-j}} \mathcal{I}_{n, i}
\end{array}\right)
$$

Using the law of total variance, this can be decomposed as:

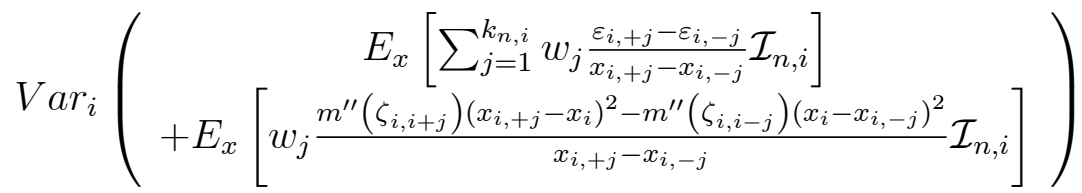

$$
\begin{aligned}
& +E_{i}\left[\operatorname{Var}_{x}\left(\sum_{j=1}^{k_{n, i}} w_{j} \frac{\varepsilon_{i,+j}-\varepsilon_{i,-j}}{x_{i,+j}-x_{i,-j}} \mathcal{I}_{n, i}\right)\right]
\end{aligned}
$$

We first note that

$$
E_{x}\left[\sum_{j=1}^{k_{n, i}} w_{j} \frac{\varepsilon_{i,+j}-\varepsilon_{i,-j}}{x_{i,+j}-x_{i,-j}} \mathcal{I}_{n, i}\right]=0
$$

since $E\left(\varepsilon_{i+j} \mid x_{i,+j}\right)=E\left(\varepsilon_{i-j} \mid x_{i,-j}\right)=0$. Thus the first term in (29) becomes:

$$
\begin{aligned}
& \operatorname{Var}_{i}\left(E_{x}\left[\sum_{j=1}^{k_{n, i}} w_{j} \frac{m^{\prime \prime}\left(\zeta_{i, i+j}\right)\left(x_{i,+j}-x_{i}\right)^{2}-m^{\prime \prime}\left(\zeta_{i, i-j}\right)\left(x_{i}-x_{i,-j}\right)^{2}}{x_{i,+j}-x_{i,-j}} \mathcal{I}_{n, i}\right]\right) \\
= & E_{i}\left[\sum_{j=1}^{k_{n, i}} w_{j} \frac{m^{\prime \prime}\left(\zeta_{i, i+j}\right)\left(x_{i,+j}-x_{i}\right)^{2}-m^{\prime \prime}\left(\zeta_{i, i-j}\right)\left(x_{i}-x_{i,-j}\right)^{2}}{x_{i,+j}-x_{i,-j}} \mathcal{I}_{n, i}\right] \\
& -E_{i}\left[\sum_{j=1}^{k_{n, i}} w_{j} \frac{m^{\prime \prime}\left(\zeta_{i, i+j}\right)\left(x_{i,+j}-x_{i}\right)^{2}-m^{\prime \prime}\left(\zeta_{i, i-j}\right)\left(x_{i}-x_{i,-j}\right)^{2}}{x_{i,+j}-x_{i,-j}} \mathcal{I}_{n, i}\right]^{2}
\end{aligned}
$$


The final term converges to zero by the preceding lemma. The first term is:

$$
\begin{aligned}
& E_{i}\left[\left(\sum_{j=1}^{k_{n, i}} w_{j} \frac{m^{\prime \prime}\left(\zeta_{i, i+j}\right)\left(x_{i,+j}-x_{i}\right)^{2}-m^{\prime \prime}\left(\zeta_{i, i-j}\right)\left(x_{i}-x_{i,-j}\right)^{2}}{x_{i,+j}-x_{i,-j}} \mathcal{I}_{n, i}\right)^{2}\right] \\
\leq & E_{i}\left[\left(\sum_{j=1}^{k_{n, i}} w_{j}\left|\frac{m^{\prime \prime}\left(\zeta_{i, i+j}\right)\left(x_{i,+j}-x_{i}\right)^{2}-m^{\prime \prime}\left(\zeta_{i, i-j}\right)\left(x_{i}-x_{i,-j}\right)^{2}}{x_{i,+j}-x_{i,-j}} \mathcal{I}_{n, i}\right|\right)^{2}\right] \\
\leq & {\left[\sup _{x} m^{\prime \prime}(x)\right]^{2} E_{i}\left[\left(\sum_{j=1}^{k_{n, i}} w_{j} \frac{\left(x_{i,+j}-x_{i}\right)^{2}+\left(x_{i}-x_{i,-j}\right)^{2}}{x_{i,+j}-x_{i,-j}} \mathcal{I}_{n, i}\right)^{2}\right] } \\
\leq & {\left[\sup _{x} m^{\prime \prime}(x)\right]^{2} E_{i}\left[\left(x_{i+k_{n, i}}-x_{i-k_{n, i}}\right)^{2} \mathcal{I}_{n, i}\right], }
\end{aligned}
$$

which approaches zero as $n \rightarrow \infty$ by Lemma 5 and almost sure boundedness. Thus, the first term in (29) converges to zero. Turning to the second term in (29):

$$
\begin{aligned}
& E_{i}\left[\operatorname{Var}_{x}\left(\sum_{j=1}^{k_{n, i}} w_{j} \frac{\varepsilon_{i,+j}-\varepsilon_{i,-j}}{x_{i,+j}-x_{i,-j}} \mathcal{I}_{n, i}\right)\right] \\
= & E_{i}\left[2 \sigma^{2} \sum_{j=1}^{k_{n, i}} \frac{w_{j}^{2}}{\left(x_{i,+j}-x_{i,-j}\right)^{2}} \mathcal{I}_{n, i}\right] \\
\leq & \frac{2 \sigma^{2}}{4 l_{n}^{2}} E_{i}\left[\sum_{j=1}^{k_{n, i}} \frac{j^{2}}{k_{n, i}^{2}\left(k_{n, i}+1\right)^{2} / 4} \mathcal{I}_{n, i}\right] \\
\leq & \frac{\sigma^{2}}{3 l_{n}^{2}} E_{i}\left[\frac{2 k_{n, i}+1}{k_{n, i}\left(k_{n, i}+1\right)} \mathcal{I}_{n, i}\right] \\
\leq & \frac{\sigma^{2}}{3} E_{i}\left[\frac{1}{l_{n}^{2} k_{n, i}} \mathcal{I}_{n, i}\right] \\
\leq & \frac{\sigma^{2}}{3}\left[\frac{1}{l_{n}^{2} \kappa_{n}}+\frac{1}{l_{n}^{2}} P\left(k_{n, i}<\kappa_{n}\right)\right]
\end{aligned}
$$

The first term in brackets approaches zero by assumption. For sufficiently large $n$, by applying $\kappa_{n} n^{-1} \rightarrow 0$ and Hoeffding's inequality as in the previous lemma, we have for sufficiently large $n>N$ :

$$
\frac{1}{l_{n}^{2}} P\left(k_{n, i}<\kappa_{n}\right) \leq \frac{1}{l_{n}^{2}} \exp \left(-\frac{(n-1) p_{N}^{2}}{2}\right)
$$

where:

$$
p_{N}=\int_{x_{\min }}^{u-l_{N}} f(s) d s+\int_{u+l_{N}}^{x_{\max }} f(s) d s .
$$


Since $l_{n}^{2} \kappa_{n} \rightarrow \infty, \kappa_{n} n^{-1} \rightarrow 0$, and $n \exp (-n) \rightarrow 0$, it follows that:

$$
\frac{1}{l_{n}^{2}} P\left(k_{n, i}<\kappa_{n}\right) \rightarrow 0
$$

as desired.

Turning to the covariance in equation (28), we apply the law of total covariance: ${ }^{44}$

$$
\begin{aligned}
& \frac{1}{n^{2}} \sum_{i \neq j} \operatorname{Cov}\left(K_{h_{n}}\left(x-x_{i}\right) \widetilde{Y}_{i}, K_{h_{n}}\left(x-x_{j}\right) \widetilde{Y}_{j}\right) \\
= & \frac{n-1}{n} E\left[K_{h_{n}}\left(x-x_{i}\right) K_{h_{n}}\left(x-x_{j}\right) \operatorname{Cov}_{i j}\left(\widetilde{Y}_{i}, \widetilde{Y}_{j}\right)\right] \\
& +\frac{n-1}{n} \operatorname{Cov}\left(K_{h_{n}}\left(x-x_{i}\right) E_{i j}\left[\widetilde{Y}_{i}\right], K_{h_{n}}\left(x-x_{j}\right) E_{i j}\left[\widetilde{Y}_{j}\right]\right) \\
\rightarrow & \frac{n-1}{n} E\left[K_{h_{n}}\left(x-x_{i}\right) K_{h_{n}}\left(x-x_{j}\right) \operatorname{Cov}_{i j}\left(\widetilde{Y}_{i}, \widetilde{Y}_{j}\right)\right] \\
& +\frac{n-1}{n} \operatorname{Cov}\left(K_{h_{n}}\left(x-x_{i}\right) m^{\prime}\left(x_{i}\right), K_{h_{n}}\left(x-x_{j}\right) m^{\prime}\left(x_{j}\right)\right) \\
= & \frac{n-1}{n} E\left[K_{h_{n}}\left(x-x_{i}\right) K_{h_{n}}\left(x-x_{j}\right) \operatorname{Cov}_{i j}\left(\widetilde{Y}_{i}, \widetilde{Y}_{j}\right)\right] .
\end{aligned}
$$

By Hardle (1990), we thus need to show $\operatorname{Cov}_{i j}\left(\widetilde{Y}_{i}, \widetilde{Y}_{j}\right)$ converges to zero as $n \rightarrow \infty$. This will be the case as long as the probability that there is overlapping data used to form the empirical derivatives $\widetilde{Y}_{i}$ and $\widetilde{Y}_{j}$ goes to zero as $n \rightarrow \infty$. This can easily be shown by Hoeffding's inequality, using the exact same approach as in Lemma 5.

The previous two lemmas show that the numerator of equation (27) converges in meansquare error to zero, which implies convergence in probability. The theorem thus follows by Slutsky's theorem.

\section{B.2 Multivariate Case}

We now extend our estimation procedure to the multidimensional case. Suppose we draw an iid sample of size $n$ from the following nonparametric model

$$
Y_{i}=m\left(\mathbf{X}_{i}\right)+\varepsilon_{i}
$$

where $E\left(\varepsilon_{i} \mid \mathbf{X}_{i}\right)=0$ and $\operatorname{Var}\left(\varepsilon_{i} \mid \mathbf{X}_{i}\right)=\sigma^{2}$. We assume that $\mathbf{X}_{i}=\left(x_{1, i}, \ldots x_{D, i}\right) \in \mathbb{R}^{D}$ and $m$ is twice continuously differentiable in all of its arguments. The variables $\mathbf{X}_{i}$ are distributed according to the continuous joint density function:

$$
f(\mathbf{X}): \prod_{d=1}^{D}\left[x_{d, \min }, x_{d, \max }\right] \rightarrow(0, \infty) .
$$

\footnotetext{
${ }^{44}$ We denote $E_{i j}[\cdot]=E\left[\cdot \mid x_{i}, x_{j}\right]$ and $\operatorname{Cov}_{i j}(\cdot)=\operatorname{Cov}\left(\cdot \mid x_{i}, x_{j}\right)$.
} 
We propose the following Nadaraya-Watson kernel estimator for the first derivative $\partial m / \partial x_{d}$ :

$$
\begin{aligned}
\frac{\partial \widehat{m}}{\partial x_{d}} & =\frac{n^{-1} \sum_{i=1}^{n} K_{\mathbf{H}_{n}}\left(\mathbf{X}-\mathbf{X}_{i}\right) \tilde{Y}_{i}}{n^{-1} \sum_{i=1}^{n} K_{\mathbf{H}_{n}}\left(\mathbf{X}-\mathbf{X}_{i}\right)} \\
\tilde{Y}_{i} & =\sum_{j=1}^{k_{n, i}} w_{j} \frac{Y_{a(j, i, d)}-Y_{b(j, i, d)}}{x_{d, a(j, i, d)}-x_{d, b(j, i, d)}} \mathbf{1}\left[k_{n, i}>0\right] \\
w_{j} & =\frac{j}{k_{n, i}\left(k_{n, i}+1\right) / 2},
\end{aligned}
$$

with the observations $\left\{\left(\mathbf{X}_{a(j, i, d)}, Y_{a(j, i, d)}\right)\right\}_{j=1}^{k_{n}}\left\{\left(\mathbf{X}_{b(j, i, d)}, Y_{b(j, i, d)}\right)\right\}_{j=1}^{k_{n}}$ defined recursively by:

$$
\begin{aligned}
& a(1, i, d)=\arg \min _{\left\{p \in L_{d, i}: x_{d, p}>x_{d, i}+l_{n}\right\}} x_{d, p}, \quad b(1, i, d)=\arg \underset{\left\{p \in L_{d, i}: x_{d, p}<x_{d, i}-l_{n}\right\}}{\max } x_{d, p} \\
& a(j, i, d)=\arg \underset{\left\{p \in L_{d, i}: x_{d, p}>x_{d, a(j-1, i, d)}\right\}}{\min } x_{d, p}, \quad b(j, i, d)=\arg \underset{\left\{p \in L_{d, i}: x_{d, p}<x_{d, b(j-1, i, d)}\right\}}{\max } x_{d, p}
\end{aligned}
$$

for $j=2, \ldots, k_{n, i}$ where $l_{n}>0$ and:

$$
L_{d, i}:=\left\{p \in\{1, \ldots, n\}: \frac{\left(x_{q, p}-x_{q, i}\right)^{2}}{\left(x_{d, p}-x_{d, i}\right)^{2}}<\vartheta_{n} \text { for all } q \neq d\right\}
$$

for some $\vartheta_{n}>0$. The random variable $k_{n, i}$ is defined as:

$$
\begin{aligned}
k_{n, i} & =\min \left(\left|L_{n, i}\right|,\left|U_{n, i}\right|, \kappa_{n}\right) \\
L_{i} & =\left\{p \in L_{d, i}: x_{d, p}>x_{d, i}+l_{n}\right\} \\
U_{i} & =\left\{p \in L_{d, i}: x_{d, p}>x_{d, i}-l_{n}\right\}
\end{aligned}
$$

for some $\kappa_{n}>0$. Letting $\mathbf{H}_{n}=\left(h_{1, n}, \ldots, h_{D, n}\right)$, note that:

$$
K_{\mathbf{H}_{n}}\left(\mathbf{X}-\mathbf{X}_{i}\right)=\frac{1}{h_{1, n} \cdots h_{D, n}} K\left(\frac{x_{1}-x_{1, i}}{h_{1, n}}, \ldots, \frac{x_{D}-x_{D, i}}{h_{D, n}}\right),
$$

where $K(\cdot)$ is a kernel function. We have the following result:

Theorem 8 Assume the random design model above and suppose:

1. $K(u)$ is bounded, compactly supported, and spherically symmetric.

2. $n \rightarrow \infty, h_{n} \rightarrow 0, l_{n} \rightarrow 0, \kappa_{n} \rightarrow \infty, \vartheta_{n} \rightarrow 0$

3. $n h_{n} \rightarrow \infty, \kappa_{n}\left(n \vartheta_{n}^{D-1}\right)^{-1} \rightarrow 0, l_{n}^{2} \kappa_{n} \rightarrow \infty$

Then:

$$
\frac{\partial \widehat{m}}{\partial x_{d}}(\mathbf{X}) \rightarrow^{p} \frac{\partial m}{\partial x_{d}}(\mathbf{X})
$$


for all $\mathbf{X} \in \prod_{d=1}^{D}\left(x_{d, \min }, x_{d, \max }\right)$

We again prove the result in a sequence of lemmas.

Lemma 9 The estimate

$$
n^{-1} \sum_{i=1}^{n} K_{\mathbf{H}_{n}}\left(\mathbf{X}-\mathbf{X}_{i}\right)
$$

converges in probability to $f(\mathbf{X})$ for all $\mathbf{X} \in \prod_{d=1}^{D}\left(x_{d, \min }, x_{d, \max }\right)$.

Proof. This is a standard result. See Hardle (1990).

Lemma 10 As $n \rightarrow \infty, P\left(k_{n, i}<\infty\right) \rightarrow 0$.

Proof. Unlike the univariate case, we have to do some work to prove this. The issue is that the "bowtie" may collapse to a line too quickly. Since $\kappa_{n} \rightarrow \infty$, without loss of generality it suffices to show that $P\left(\left|U_{n, i}\right|<\infty\right) \rightarrow 0$. We first note that since $f$ is a continuous function from a compact set to $(0, \infty)$ it must achieve a minimum, which we denote as $\Delta>0$. Choose any $M \geq 0$. It is then straightforward to check via multiple integration that for $n>M+1$ :

$$
P\left(\left|U_{n, i}\right| \leq M\right) \leq F_{B}\left(M ; n-1, p_{\Delta}\right)
$$

where:

$$
p_{\Delta}=\frac{\Delta \vartheta_{n}^{(D-1) / 2}\left(x_{d, \max -} x_{d, i}\right)^{D}}{D !}
$$

By Hoeffding's inequality:

$$
\begin{aligned}
P\left(\left|U_{n, i}\right| \leq M\right) & \leq \exp \left(-2 \frac{\left((n-1) p_{\Delta}-M\right)^{2}}{n-1}\right) \\
& =\exp \left(-2\left[(n-1) p_{\Delta}^{2}-2 p_{\Delta} M+\frac{M^{2}}{n-1}\right]\right) .
\end{aligned}
$$

Since $\kappa_{n}\left(n \vartheta_{n}^{D-1}\right)^{-1} \rightarrow 0$, it must be that $n \vartheta_{n}^{D-1} \rightarrow \infty$. It therefore follows that $(n-1) p_{\Delta}^{2} \rightarrow$ $\infty$. Two two final terms in the bracket approach zero. Therefore, $P\left(\left|U_{n, i}\right| \leq M\right)$ converges to zero.

Lemma 11 As $n \rightarrow \infty$, the bias:

$$
\left|E\left[n^{-1} \sum_{i=1}^{n} K_{\mathbf{H}_{n}}\left(\mathbf{X}-\mathbf{X}_{i}\right) \widetilde{Y}_{i}\right]-\frac{\partial m}{\partial x_{d}}(\mathbf{X}) f(\mathbf{X})\right| \rightarrow 0
$$


Proof. Write the bias as:

$$
\begin{aligned}
& \left|E\left[\frac{1}{n} \sum_{i=1}^{n} K_{\mathbf{H}_{n}}\left(\mathbf{X}-\mathbf{X}_{i}\right) \tilde{Y}_{i}\right]-\frac{\partial m}{\partial x_{d}}(\mathbf{X}) f(\mathbf{X})\right| \\
= & \left|E\left[\sum_{i=1}^{n} K_{\mathbf{H}_{n}}(\mathbf{X}-\mathbf{U}) \sum_{j=1}^{k_{n}} w_{j} \frac{Y_{a(j, i, d)}-Y_{b(j, i, d)}}{x_{d, a(j, i, d)}-x_{d, b(j, i, d)}} \mathcal{I}_{n, i}\right]-\frac{\partial m}{\partial x_{d}}(\mathbf{X}) f(\mathbf{X})\right|
\end{aligned}
$$

where $\mathbf{U}=\left(u_{1}, \ldots, u_{D}\right)$ and $d \mathbf{U}=d u_{1} \cdots d u_{D}$. Conditioning on the sample and applying Taylor's theorem as in the univariate case, we have:

$$
\begin{aligned}
& E_{\mathbf{X}}\left[\sum_{j=1}^{k_{n}} w_{j} \frac{Y_{a(j, i, d)}-Y_{b(j, i, d)}}{x_{d, a(j, i, d)}-x_{d, b(j, i, d)}} \mathcal{I}_{n, i}\right] \\
= & \sum_{j=1}^{k_{n}} \sum_{q=1}^{D} w_{j} \frac{\partial m}{\partial x_{q}}(\mathbf{U}) \frac{x_{q, a(j, i, d)}-x_{q, b(j, i, d)}}{x_{d, a(j, i, d)}-x_{d, b(j, i, d)}} \mathcal{I}_{n, i} \\
& +\sum_{j=1}^{k_{n}} \sum_{|\alpha|=2} w_{j} \frac{R_{\alpha}\left(\mathbf{X}_{a(j, i, d)}\right)\left(\mathbf{X}_{a(j, i, d)}-\mathbf{X}_{i}\right)^{\alpha}-R_{\alpha}\left(\mathbf{X}_{b(j, i, d)}\right)\left(\mathbf{X}_{b(j, i, d)}-\mathbf{X}_{i}\right)^{\alpha}}{x_{d, a(j, i, d)}-x_{d, b(j, i, d)}} \mathcal{I}_{n, i}
\end{aligned}
$$

where we have used the multi-index notation with $\alpha \in \mathbb{N}^{D}$ and where $R_{\alpha}(\cdot)$ denotes the remainder of the Taylor expansion. By the triangle inequality, the bias is therefore bounded above by

$$
\begin{aligned}
& \left|E\left[K_{\mathbf{H}_{n}}(\mathbf{X}-\mathbf{U}) \frac{\partial m}{\partial x_{d}}(\mathbf{U}) \mathcal{I}_{n, i}\right]-\frac{\partial m}{\partial x_{d}}(\mathbf{X}) f(\mathbf{X})\right| \\
& +\left|\int K_{\mathbf{H}_{n}}(\mathbf{X}-\mathbf{U}) \sum_{j=1}^{k_{n}} \sum_{q \neq d} \frac{\partial m}{\partial x_{q}}(\mathbf{U}) \sqrt{\vartheta_{n}} \mathcal{I}_{n, i} f(\mathbf{U}) d \mathbf{U}\right| \\
& +\left|\int K_{\mathbf{H}_{n}}(\mathbf{X}-\mathbf{U}) E_{i}\left[\sum_{j=1}^{k_{n}} \sum_{|\alpha|=2} w_{j} \frac{R_{\alpha}\left(\mathbf{X}_{a(j, i, d)}\right)\left(\mathbf{X}_{a(j, i, d)}-\mathbf{X}_{i}\right)^{\alpha}-R_{\alpha}\left(\mathbf{X}_{b(j, i, d)}\right)\left(\mathbf{X}_{b(j, i, d)}-\mathbf{X}_{i}\right)^{\alpha}}{x_{d, a(j, i, d)}-x_{d, b(j, i, d)}} \mathcal{I}_{n, i} f(\mathbf{U}) d \mathbf{U}\right]\right|
\end{aligned}
$$

The first term converges to zero by the usual consistency proof for multivariate NadarayaWatson estimates and since $P\left(k_{n, i}>0\right) \rightarrow 1$. We can bound the remainder according to the Taylor uniform bound. The second term converges to zero since $\vartheta_{n} \rightarrow 0$ as $n \rightarrow \infty$. The final term is therefore bounded above by:

$$
\begin{aligned}
\sup _{|\alpha|=2} \sup _{\mathbf{X}}\left|D^{\alpha} m(\mathbf{X})\right| \int\left|K_{\mathbf{H}_{n}}(\mathbf{X}-\mathbf{U})\right| E_{i}\left[\sum_{j=1}^{k_{n}} \sum_{|\alpha|=2} w_{j} \frac{\left(\mathbf{X}_{a(j, i, d)}-\mathbf{X}_{i}\right)^{\alpha}+\left(\mathbf{X}_{b(j, i, d)}-\mathbf{X}_{i}\right)^{\alpha}}{x_{d, a(j, i, d)}-x_{d, b(j, i, d)}} \mathcal{I}_{n, i}\right] f(\mathbf{U}) d \mathbf{U} \\
\leq \sup _{|\alpha|=2} \sup _{\mathbf{X}}\left|D^{\alpha} m(\mathbf{X})\right| \int\left|K_{\mathbf{H}_{n}}(\mathbf{X}-\mathbf{U})\right| E_{i}\left[\begin{array}{c}
\sum_{j=1}^{k_{n}} w_{j}\left(1+(D-1) \sqrt{\vartheta_{n}}+D(D-1) \vartheta_{n}\right) \\
\times\left(x_{d, a(j, i, d)}-x_{d, b(j, i, d)}\right) \mathcal{I}_{n, i}
\end{array}\right] f(\mathbf{U}) d \mathbf{U},
\end{aligned}
$$

which converges to zero by Hoeffding's inequality as in the univariate case and since $\vartheta_{n} \rightarrow 0$ as $n \rightarrow \infty$. 
Lemma 12 As $n \rightarrow \infty$, the variance:

$$
\operatorname{Var}\left(n^{-1} \sum_{i=1}^{n} K_{\mathbf{H}_{n}}\left(\mathbf{X}-\mathbf{X}_{i}\right) \widetilde{Y}_{i}\right) \rightarrow 0
$$

Proof. The proof follows exactly as in the univariate case, applying the multidimensional Taylor formula in place of the unidimensional Taylor formula.

The numerator of equation (30) converges in mean-square error to zero, which implies convergence in probability. The theorem follows by Slutsky's theorem.

\section{B.3 Proof of Theorem 1}

Given the previous results, the proof is straightforward. The only complication is that 2-dimensional (only in $r$ and $t$ ) smoothing is used in the Nadaraya-Watson estimator, rather than 3-dimensional smoothing. It is straightforward, however, from the work above to see that: 45

$$
\begin{aligned}
\widehat{\Phi}_{l}\left(r, T_{l}+\tau\right) & \rightarrow p \frac{\partial \widetilde{m}_{\mathbf{Y}}(R, \tau)}{\partial R}+\int_{0}^{2 \pi} \frac{\partial \phi_{l}(r, \theta)}{\partial r} f\left(\theta \mid T_{l}+\tau, r\right) d \theta \\
\widehat{\Phi}_{l}(r, T-1 \tau) & \rightarrow p \int_{0}^{2 \pi} \frac{\partial \phi_{l}(r, \theta)}{\partial r} f\left(\theta \mid T_{l}-1, r\right) d \theta
\end{aligned}
$$

By the assumption in footnote 18, $f\left(\theta \mid T_{l}+\tau, r\right)=f\left(\theta \mid T_{l}-1, r\right)$, so:

$$
\widehat{\Phi}_{l}\left(r, T_{l}+\tau\right)-\widehat{\Phi}_{l}\left(r, T_{l}-1\right) \rightarrow^{p} \frac{\partial \widetilde{m}_{\mathbf{Y}}(R, \tau)}{\partial R}
$$

as desired.

\footnotetext{
${ }^{45}$ Recall that we assume $\frac{\partial \widetilde{m}_{\mathbf{Y}}(R,-1)}{\partial R}=0$.
} 